\title{
Electrochemical Synthesis of Dimeric 2-Oxindole Sharing Vicinal Quaternary Centers Employing Proton-Coupled Electron Transfer (PCET)
}

Sulekha Sharma, ${ }^{a}$ Avishek Roy, ${ }^{a}$ Kundan Shaw, ${ }^{a}$ Alakesh Bisai, ${ }^{* a, b}$ and Amit Paul, ${ }^{* a}$

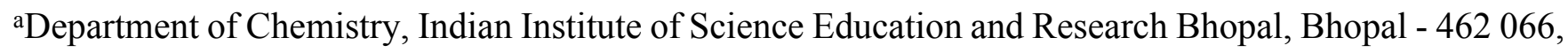
MP, India

bDepartment of Chemical Sciences, Indian Institute of Science Education and Research Kolkata, Mohanpur, Nadia - 741246, WB, India

E-Mail: apaul@iiserb.ac.in (A.P.) \& alakesh@iiserkol.ac.in (A.B.)

Table of Contents

Substrate Preparation

Cyclic Voltammetry data

S3-S5

Spectra Data

S6-S69 


\section{Substrate preparation for the oxidative dimerization:}

Compounds $1 \mathrm{a}-1 \mathrm{j},{ }^{1} 1 \mathrm{k}-1 \mathrm{~m},{ }^{2} 1 \mathrm{n}^{3}, 1 \mathrm{o}-1 \mathrm{u}^{1}$ were synthesized following literature protocols<smiles>COC(=O)C1C(=O)Nc2ccccc21</smiles>

(1a) $\mathrm{Me}$<smiles>C=CCN1C(=O)C(C(=O)OC)c2ccccc21</smiles><smiles>C=CCOC(=O)C1C(=O)N([14CH3])c2ccccc21</smiles><smiles>C=CCN1C(=O)C(c2ccccc2OC)c2ccccc21</smiles><smiles>CCOC(=O)C1C(=O)Nc2ccc(Br)cc21</smiles>

(1q) $\mathrm{Bn}$<smiles>COC(=O)C1C(=O)Nc2ccc(Cl)cc21</smiles>

(1b) $\mathrm{Me}$<smiles>C=CCOC(=O)C1C(=O)Nc2ccccc21</smiles>

(1f)<smiles>C=C(C)COC(=O)C1C(=O)N(C)c2ccccc21</smiles><smiles>COC(=O)C1C(=O)Nc2ccc(Br)cc21</smiles>

(1c) $\mathrm{Me}$<smiles>C=CCOC(=O)C1C(=O)N(C)c2ccc(Cl)cc21</smiles>

(1g)<smiles>COc1ccc(C)cc1C1C(=O)N(C)c2ccccc21</smiles>

(1k)<smiles>C=CCOC(=O)C1C(=O)N([PH](C)(=O)=O)c2ccccc21</smiles>

(1h)<smiles>C=CCN1C(=O)C(c2cc(C)ccc2OC)c2ccccc21</smiles><smiles>COC(=O)C1C(=O)N(C)c2ccccc21</smiles>

(1d) $\mathrm{PMB}$<smiles>CN1C(=O)C(c2ccccc2[N+](=O)[O-])c2ccccc21</smiles><smiles>COC(=O)C1C(=O)N(C)c2ccc(OC)cc21</smiles>

(10) $\mathrm{Me}$<smiles>CCOC(=O)C1C(=O)Nc2ccccc21</smiles>

(1s) $\mathrm{Bn}$<smiles>COC(=O)C1C(=O)N(Cc2ccccc2)c2ccc(Br)cc21</smiles>

(1p) $\mathrm{Bn}$<smiles>COC(=O)C1C(=O)N(c2ccccc2)c2ccccc21</smiles>

(1r) $\mathrm{Bn}$<smiles>COC(=O)C1C(=O)Nc2ccc(Cl)cc21</smiles>

(1t) $\mathrm{Bn}$<smiles>CCOC(=O)C1C(=O)Nc2ccc(Cl)cc21</smiles>

(1u) $\mathrm{Bn}$ 


\section{References:}

1. Ghosh, S.; Chaudhuri, S.; Bisai, A., Oxidative Dimerization of 2-Oxindoles Promoted by $\mathrm{KO}^{t} \mathrm{Bu}$

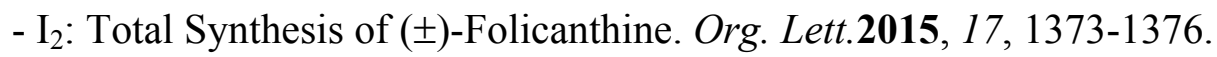

2. Maity, A.; Roy, A.; Das, M. K.; De, S.; Naskar, M.; Bisai, A., Oxidative cyanation of 2-oxindoles: formal total synthesis of $( \pm)$-gliocladin C. Org. Biomol. Chem.2020, 18, 1679-1684.

3. Roy, A.; Das, M. K.; Chaudhuri, S.; Bisai, A., Transition-Metal Free Oxidative Alkynylation of 2-Oxindoles with Ethynylbenziodoxolone (EBX) Reagents. J. Org. Chem.2018, 83, 403-421.

\section{CV data:}

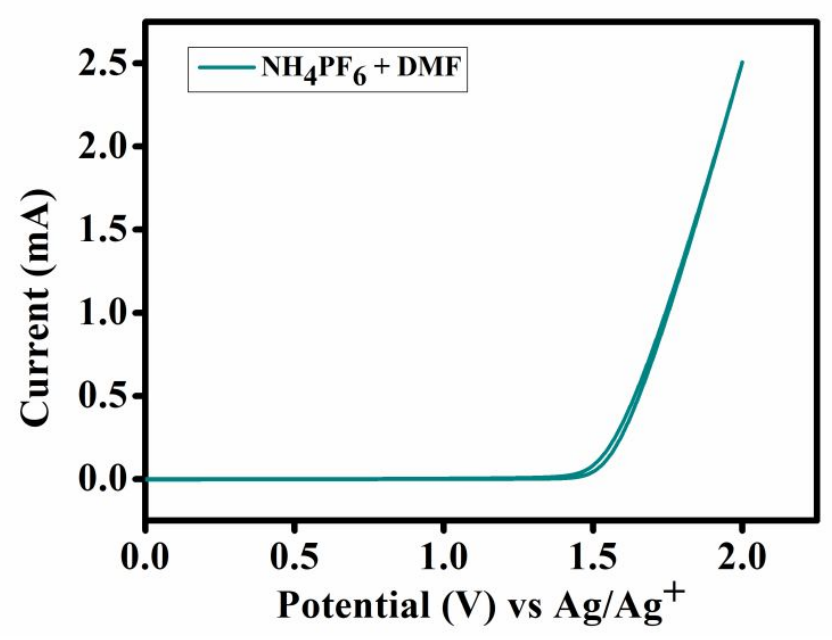

Figure S1: Cyclic Voltammogram at $100 \mathrm{mVs}^{-1}$ scan rate on glassy carbon electrode of $0.1 \mathrm{M} \mathrm{NH}_{4} \mathrm{PF}_{6}$ in DMF. 


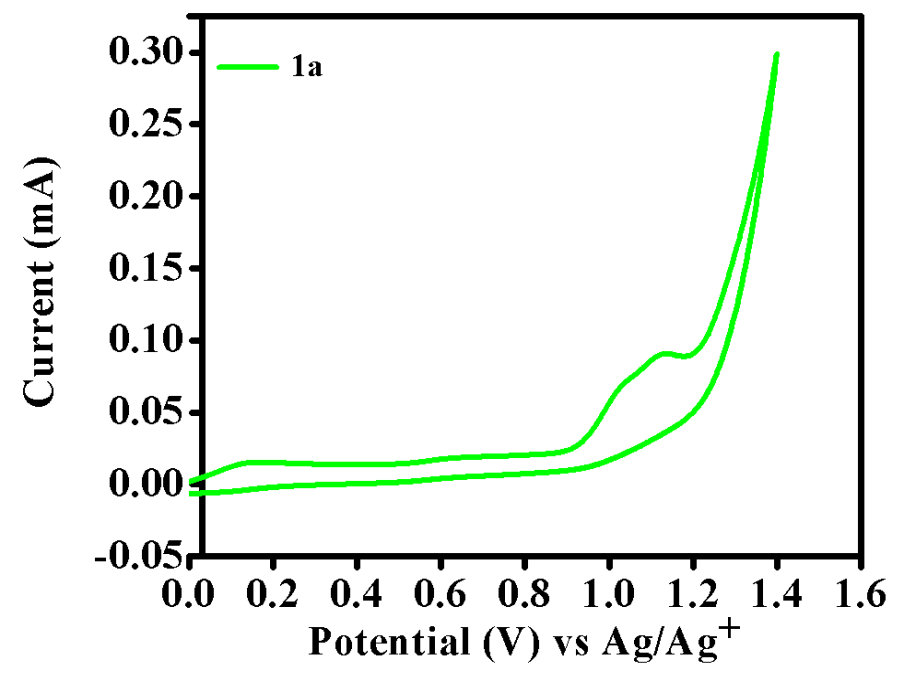

Figure S2: Cyclic Voltammogram at $100 \mathrm{mVs}^{-1}$ scan rate on glassy carbon electrode in $0.1 \mathrm{M} \mathrm{NH}_{4} \mathrm{PF}_{6}$ in DMF; $1 \mathbf{a}(5 \mathrm{mM})$; in presence of $\mathrm{N}_{2}$.

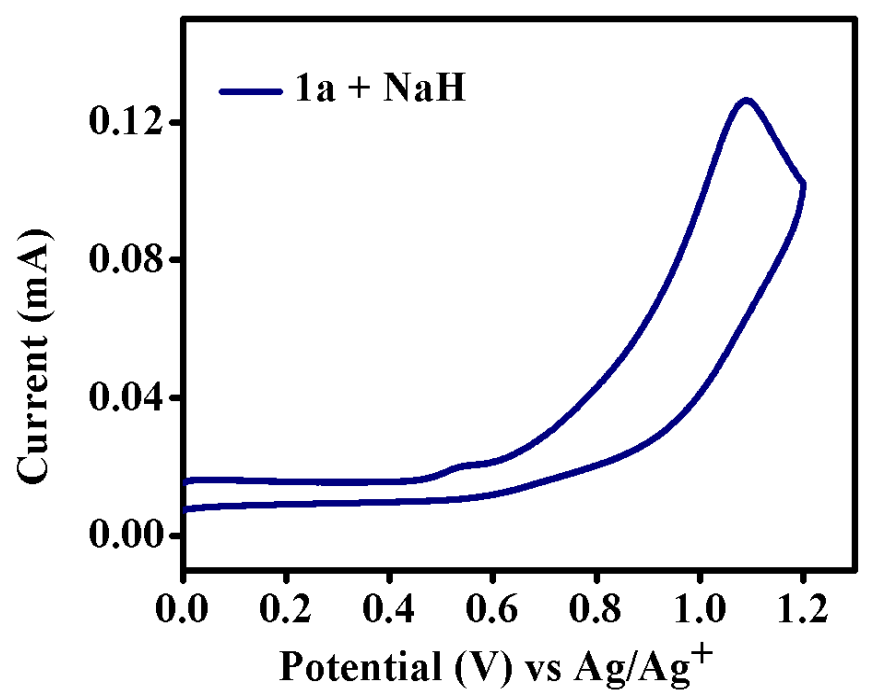

Figure S3: Cyclic Voltammogram at $100 \mathrm{mVs}^{-1}$ scan rate on glassy carbon electrode in $0.1 \mathrm{M} \mathrm{NH}_{4} \mathrm{PF}_{6}$ in $\mathrm{DMF} ; \mathbf{1 a}(5 \mathrm{mM}) ; \mathrm{NaH}(5 \mathrm{mM})$ in presence of $\mathrm{N}_{2}$. 


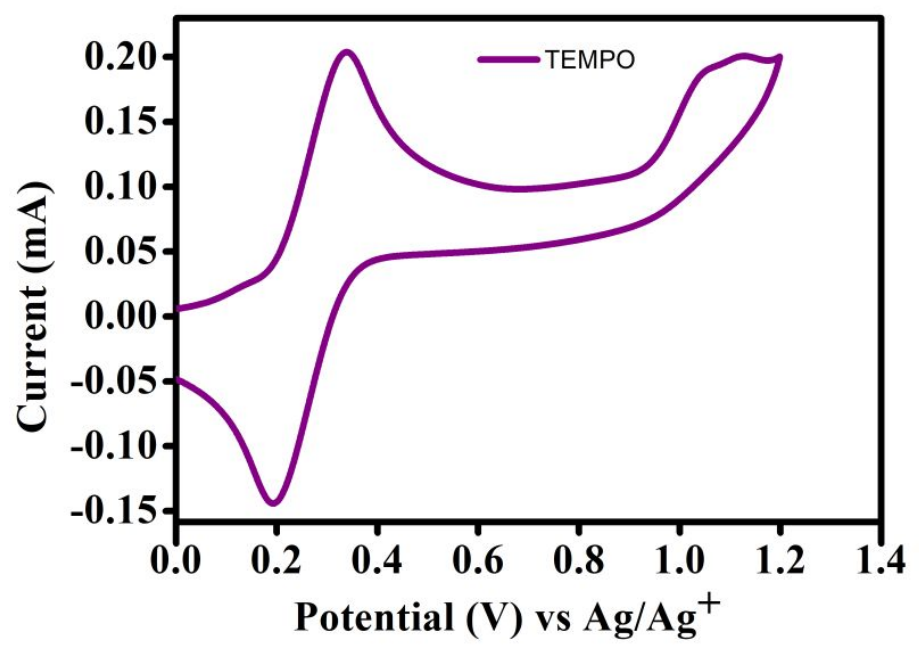

Figure S4: Cyclic Voltammogram at $100 \mathrm{mVs}^{-1}$ scan rate on glassy carbon electrode in $0.1 \mathrm{M} \mathrm{NH}_{4} \mathrm{PF}_{6}$ in DMF; 1a (5 mM); TEMPO (10 mM) in presence of $\mathrm{N}_{2}$ (blue). 


\section{Spectral Data}
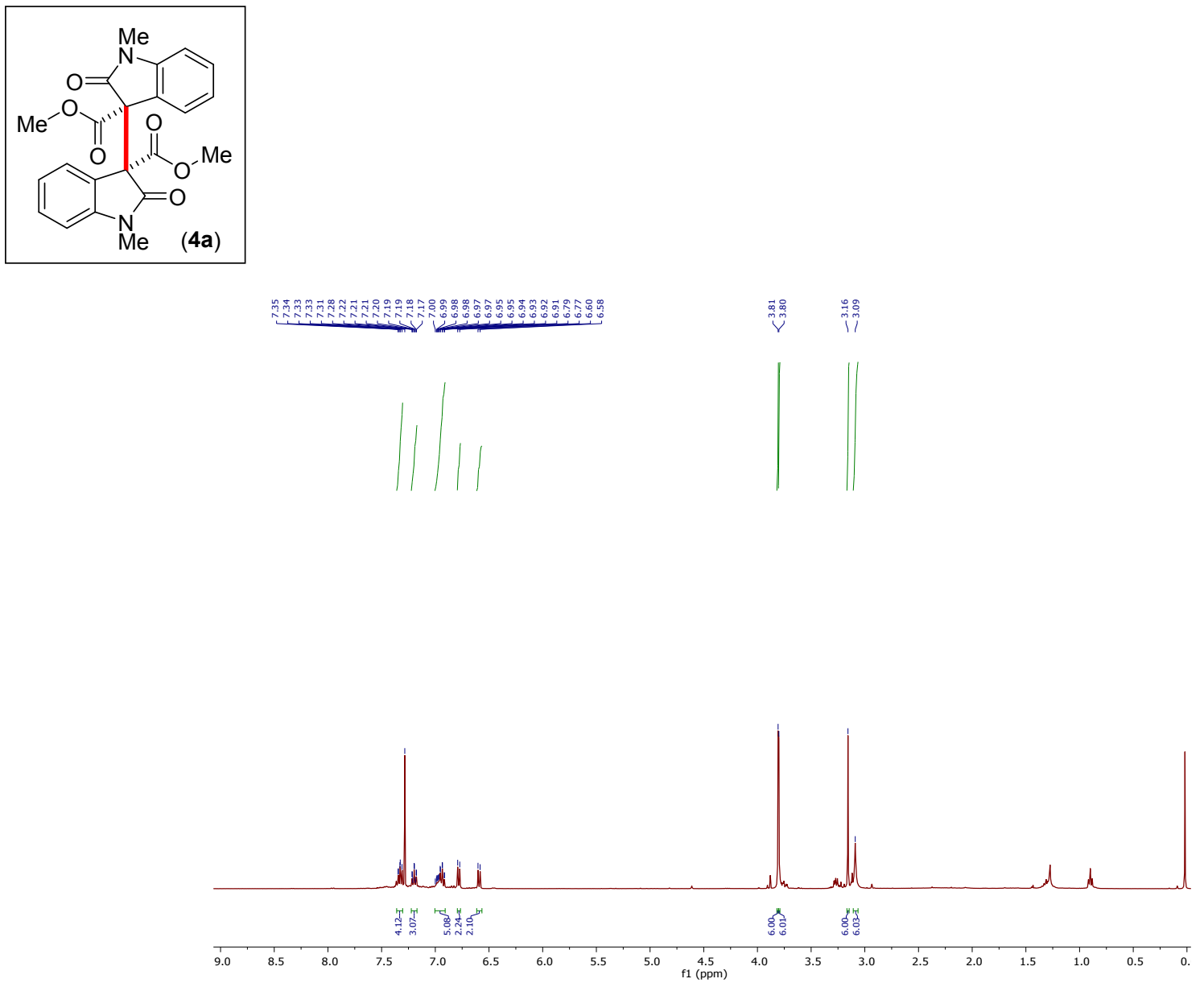

${ }^{1} \mathrm{H} \mathrm{NMR}\left(400 \mathrm{MHz}, \mathrm{CDCl}_{3}\right)$ of compound $4 \mathbf{a}$
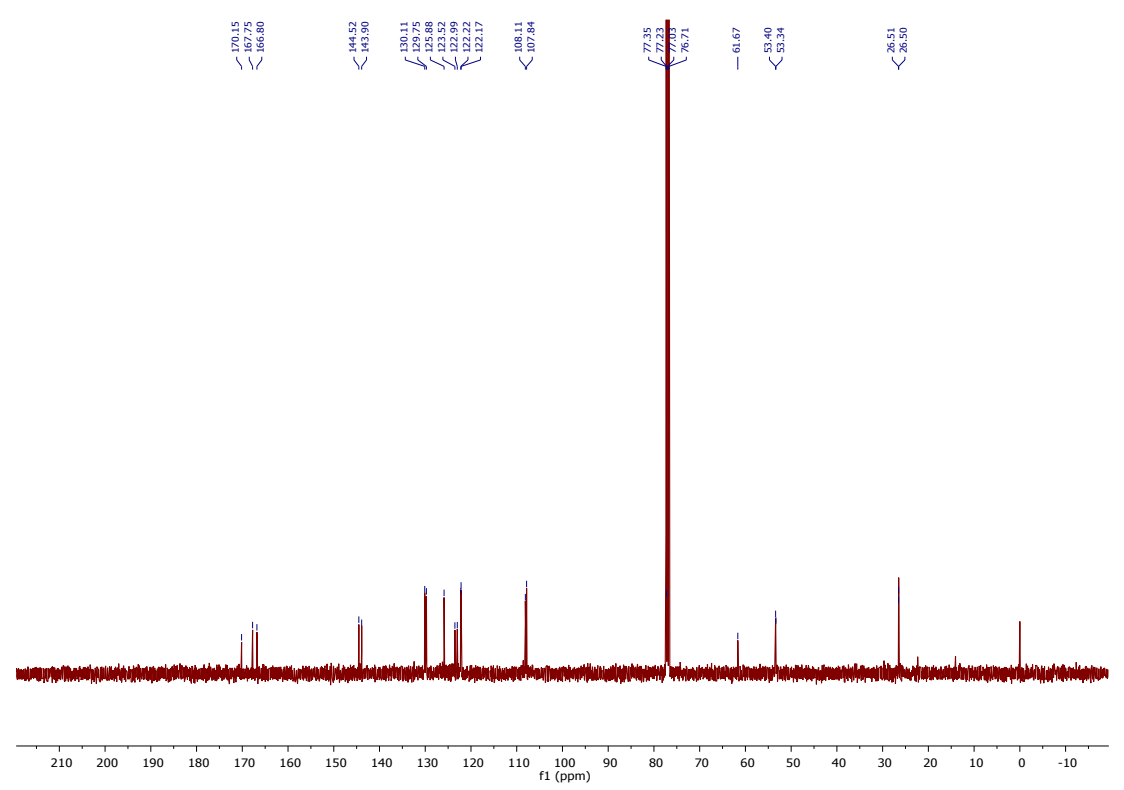

${ }^{13} \mathrm{C}\left\{{ }^{1} \mathrm{H}\right\} \mathrm{NMR}\left(100 \mathrm{MHz}, \mathrm{CDCl}_{3}\right)$ of compound 4a 


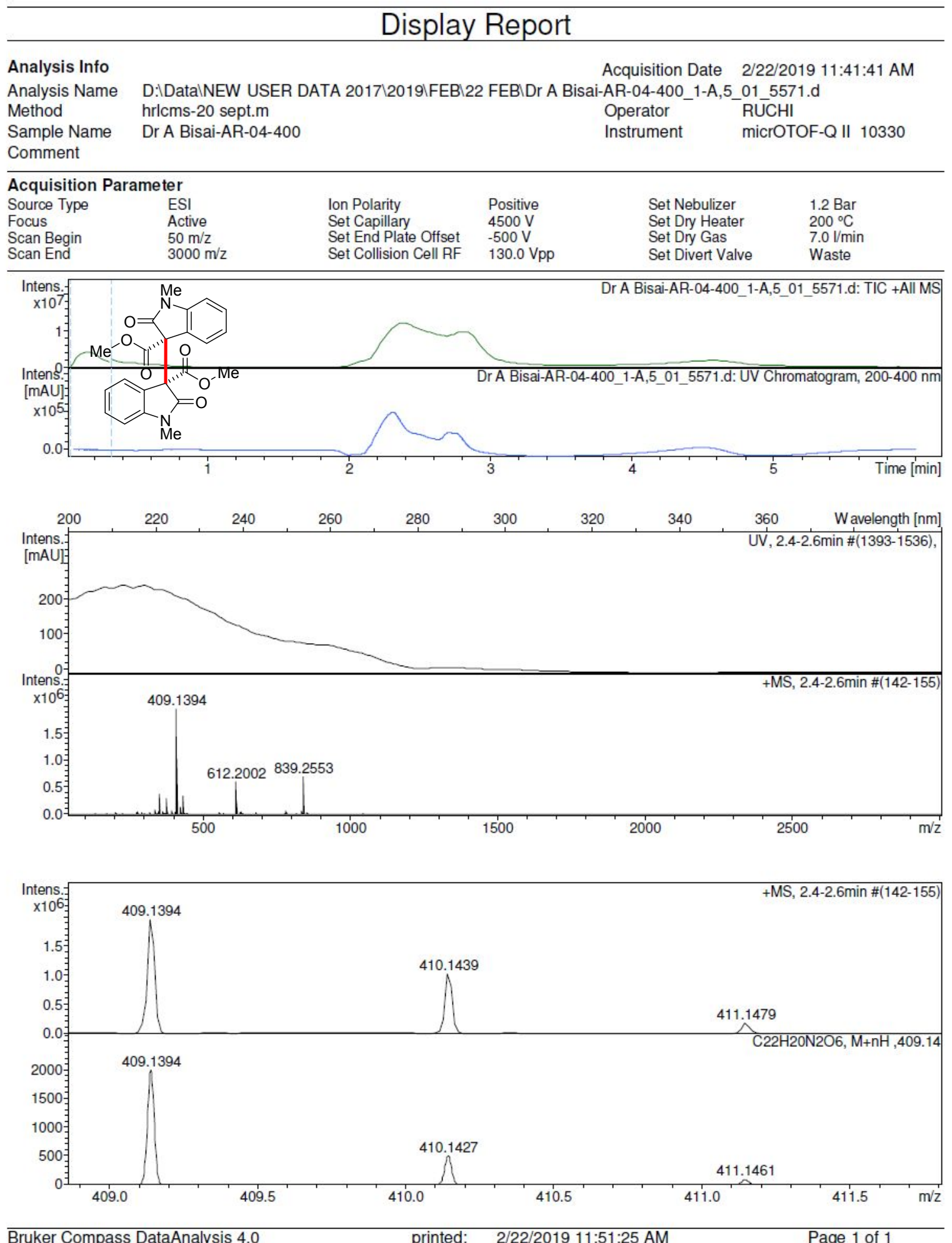

Bruker Compass DataAnalysis 4.0

printed: $\quad$ 2/22/2019 11:51:25 AM

Page 1 of 1

\section{Mass Spectrum of $\mathbf{4 a}$}



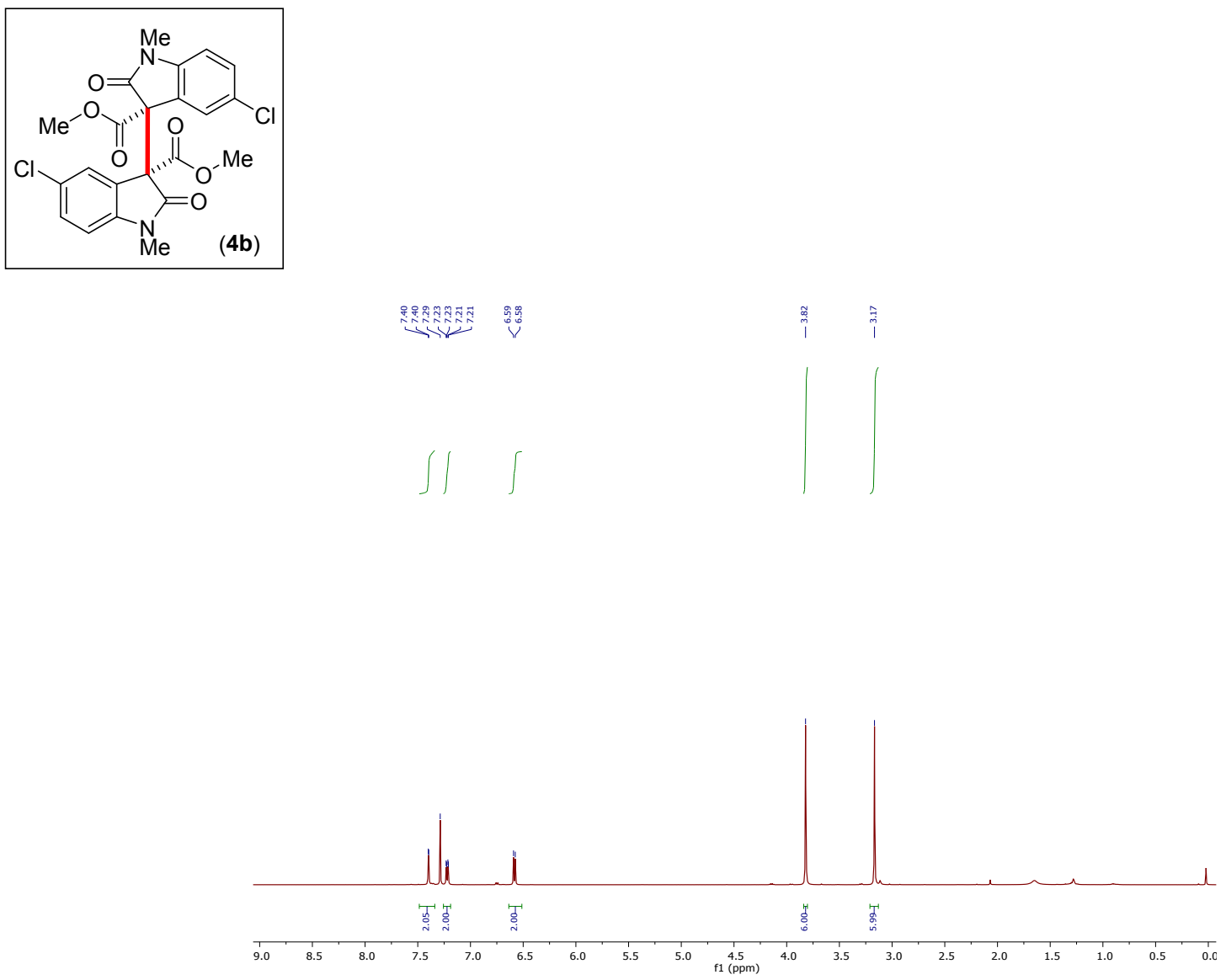

${ }^{1} \mathrm{H} \mathrm{NMR}\left(500 \mathrm{MHz}, \mathrm{CDCl}_{3}\right)$ of compound $\mathbf{4 b}$
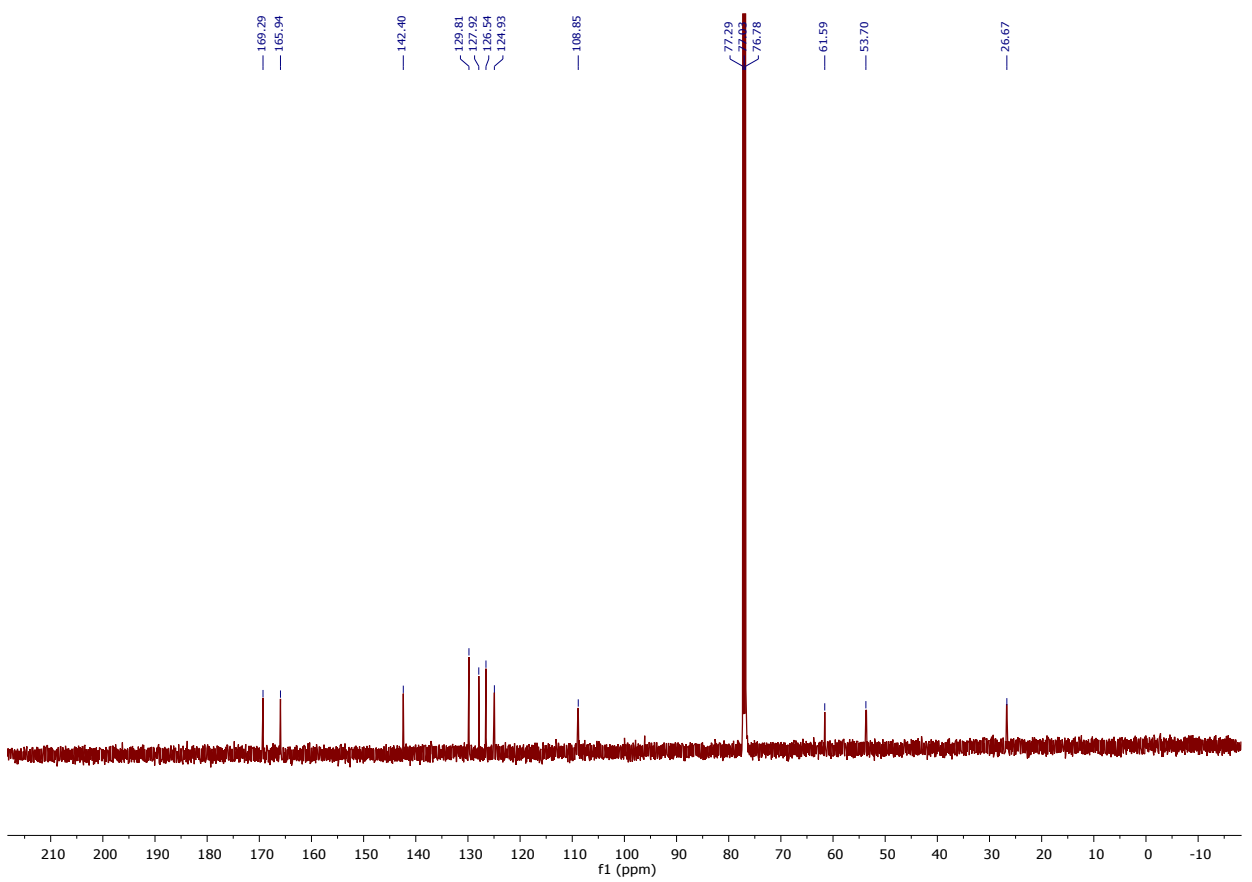

${ }^{13} \mathrm{C}\left\{{ }^{1} \mathrm{H}\right\}$ NMR (125 MHz, $\left.\mathrm{CDCl}_{3}\right)$ of compound $\mathbf{4 b}$ 


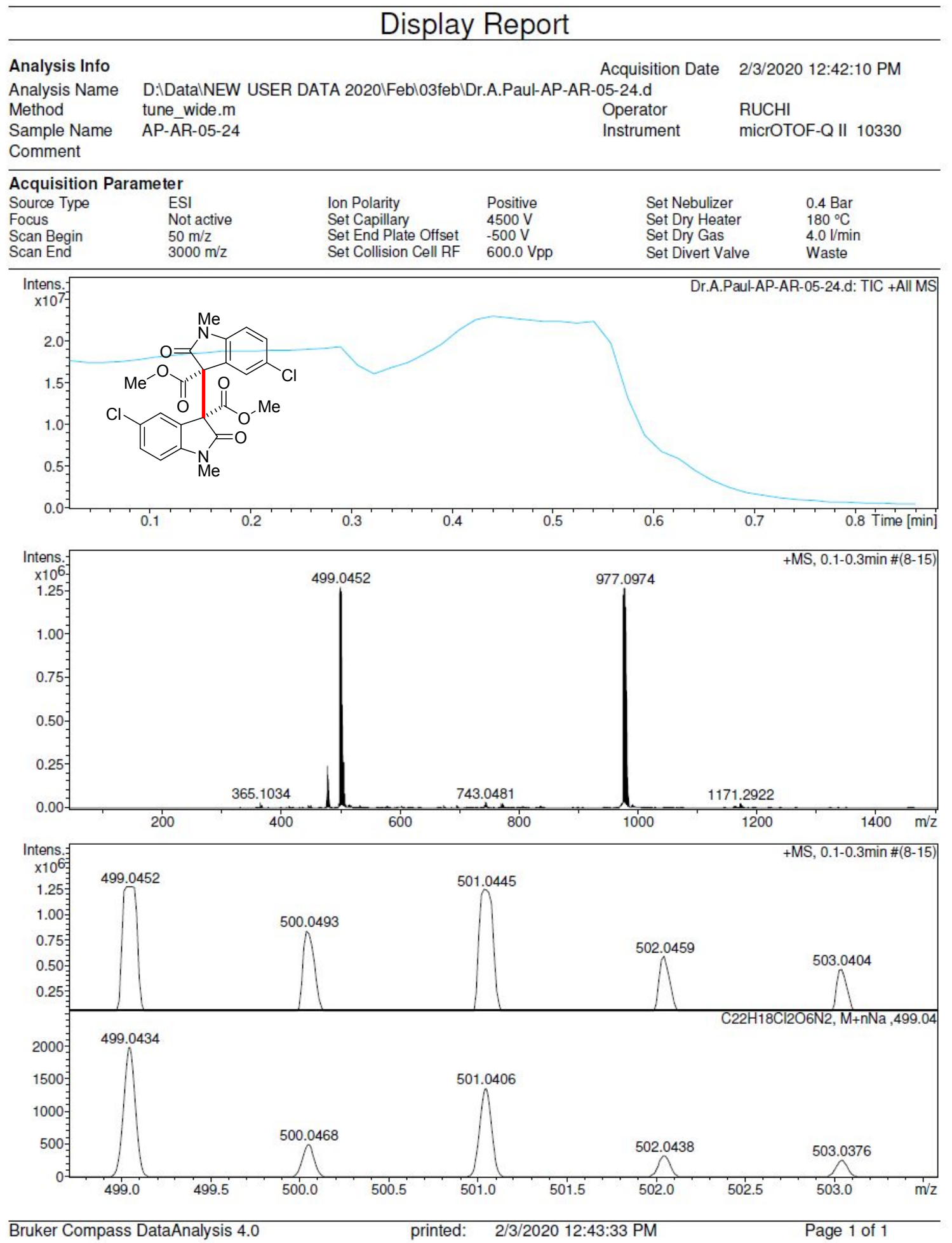

\section{Mass Spectrum of $\mathbf{4 b}$}



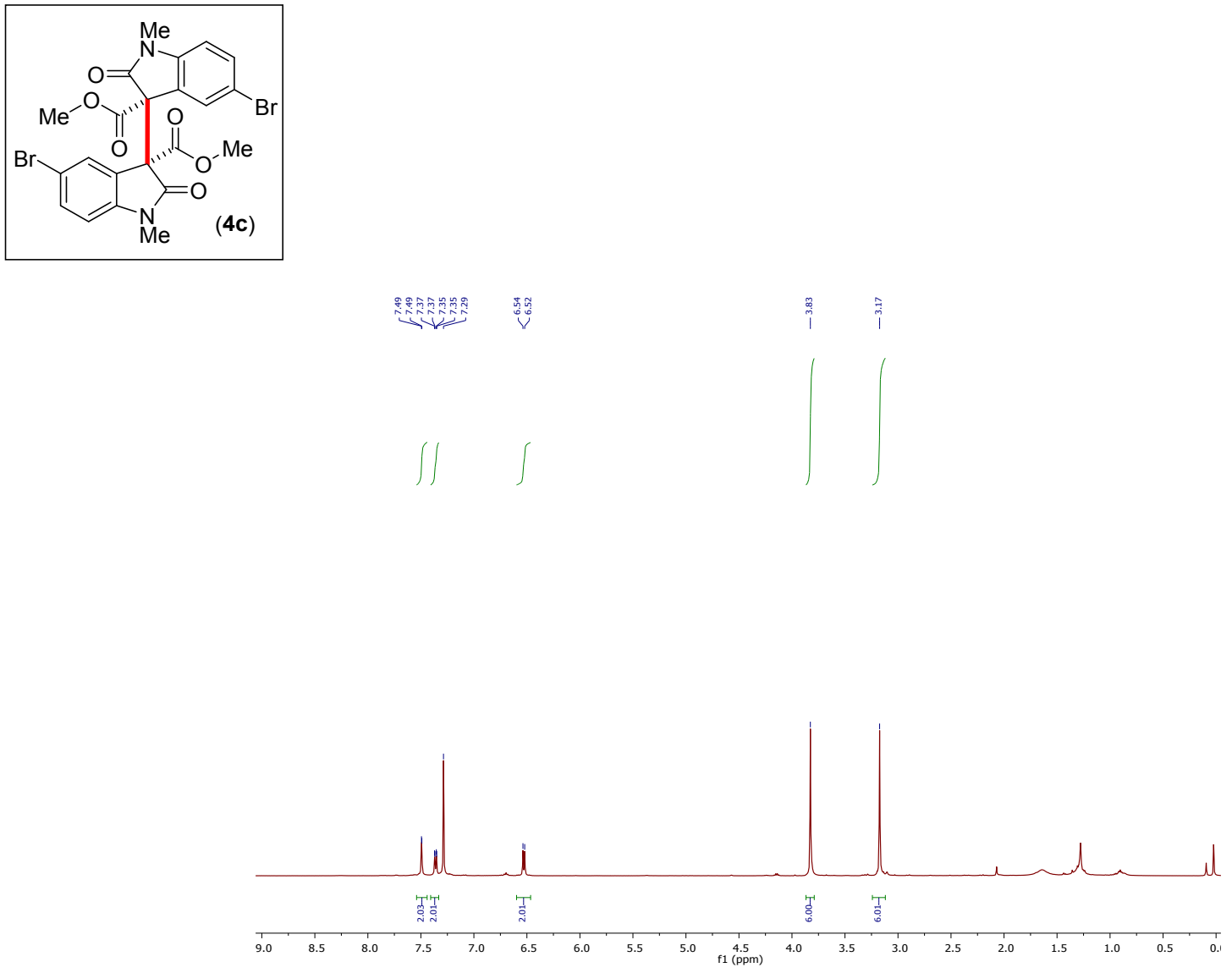

${ }^{1} \mathrm{H} \mathrm{NMR}\left(500 \mathrm{MHz}, \mathrm{CDCl}_{3}\right)$ of compound 4c
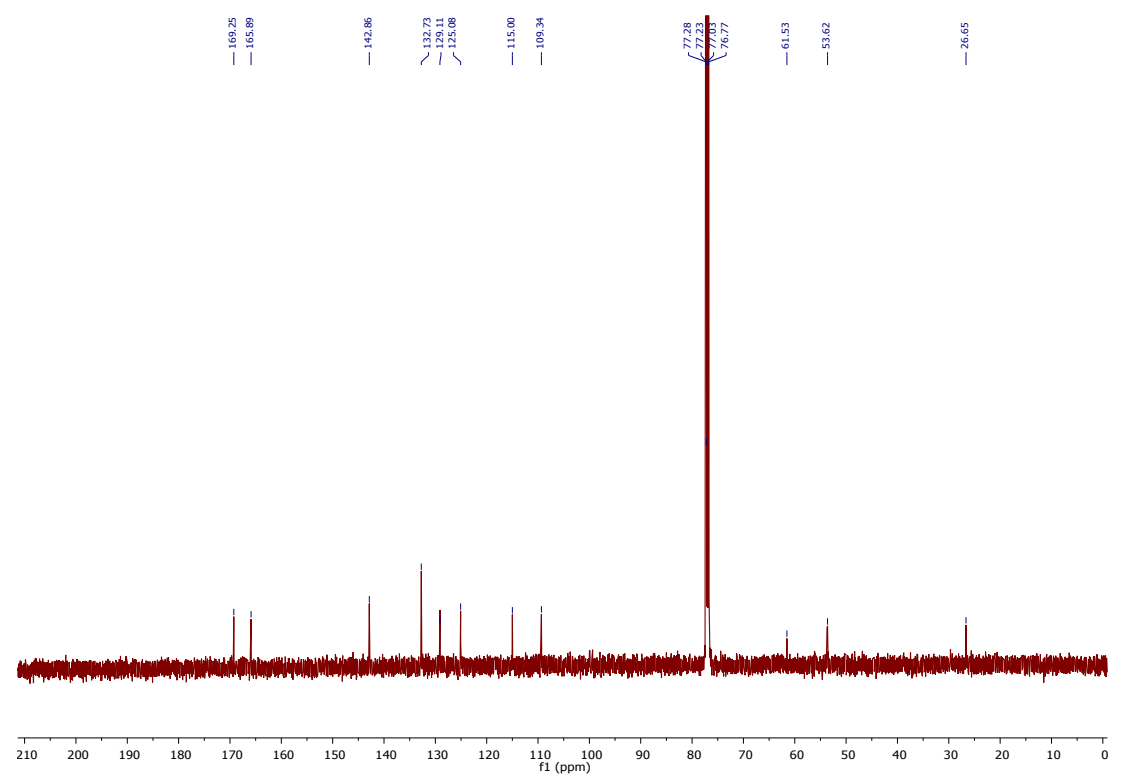

${ }^{13} \mathrm{C}\left\{{ }^{1} \mathrm{H}\right\}$ NMR $\left(125 \mathrm{MHz}, \mathrm{CDCl}_{3}\right)$ of compound $4 \mathbf{c}$ 


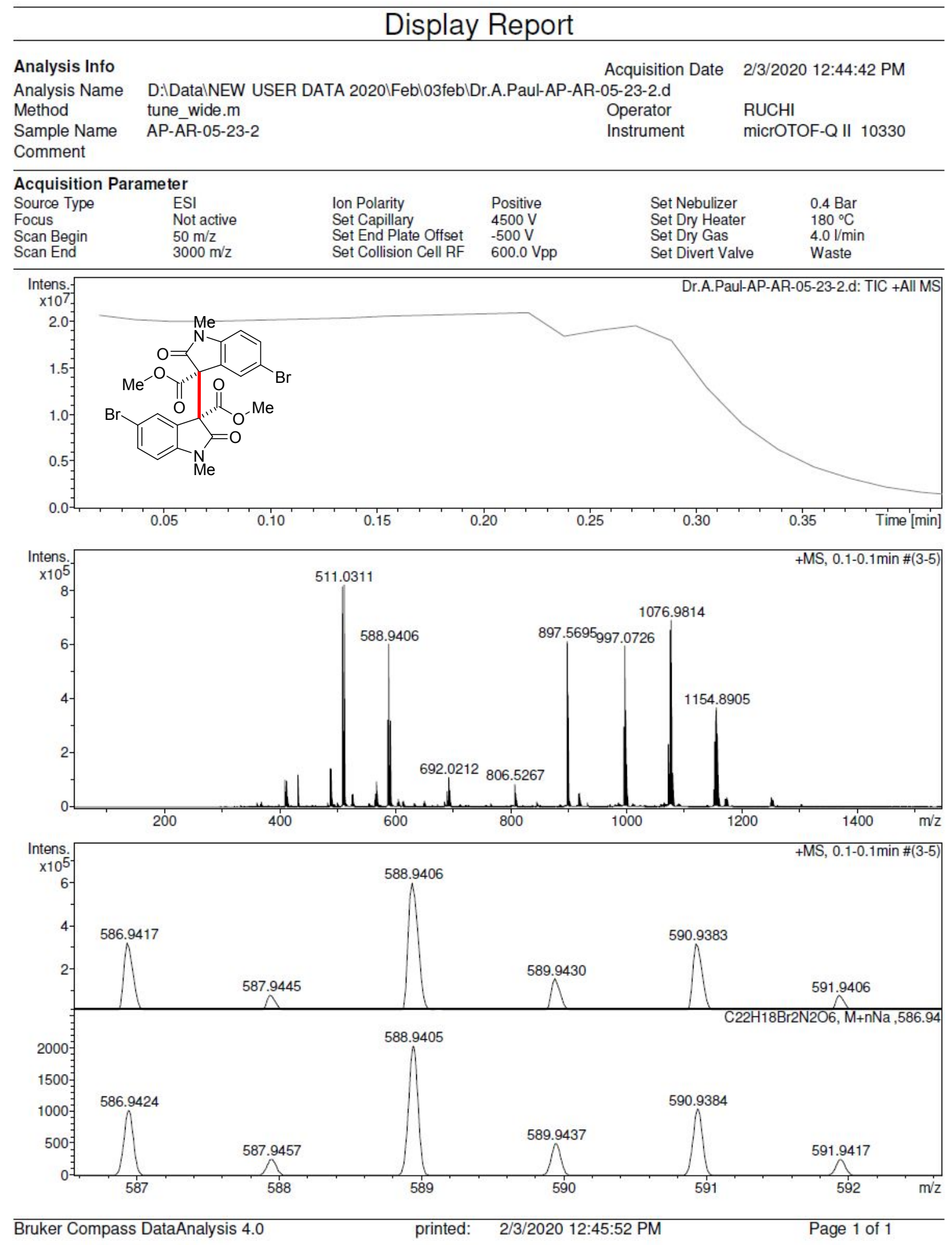

\section{Mass Spectrum of $\mathbf{4 c}$}



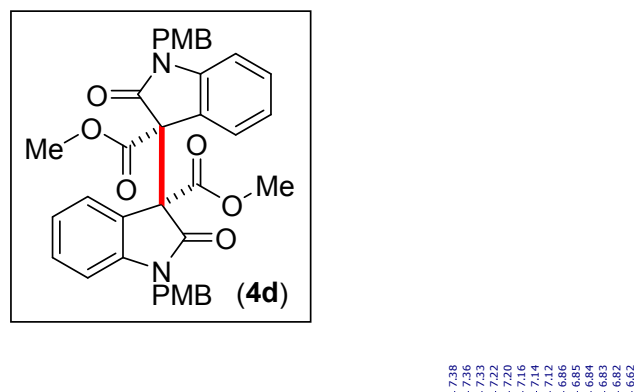

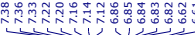

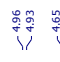

:
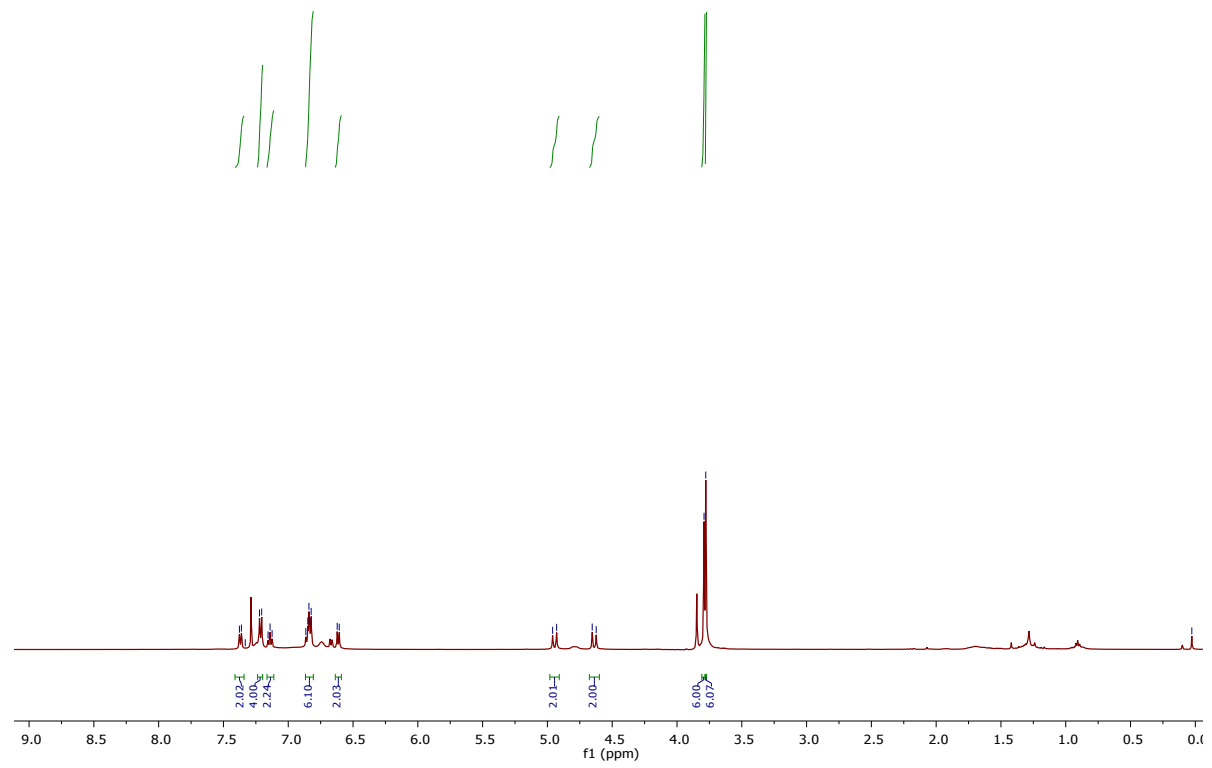

${ }^{1} \mathrm{H}$ NMR (400 MHz, $\mathrm{CDCl}_{3}$ ) of compound 4d
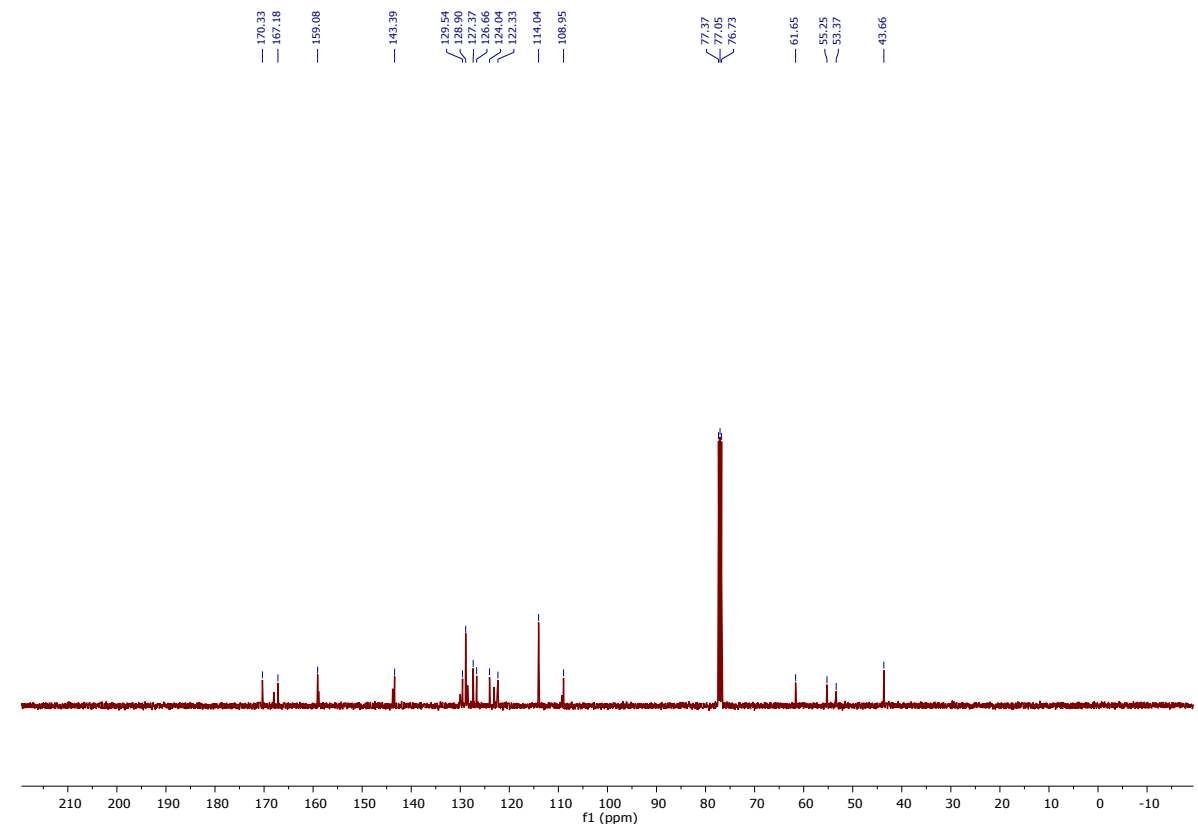

${ }^{13} \mathrm{C}\left\{{ }^{1} \mathrm{H}\right\} \mathrm{NMR}\left(100 \mathrm{MHz}, \mathrm{CDCl}_{3}\right)$ of compound 4d 


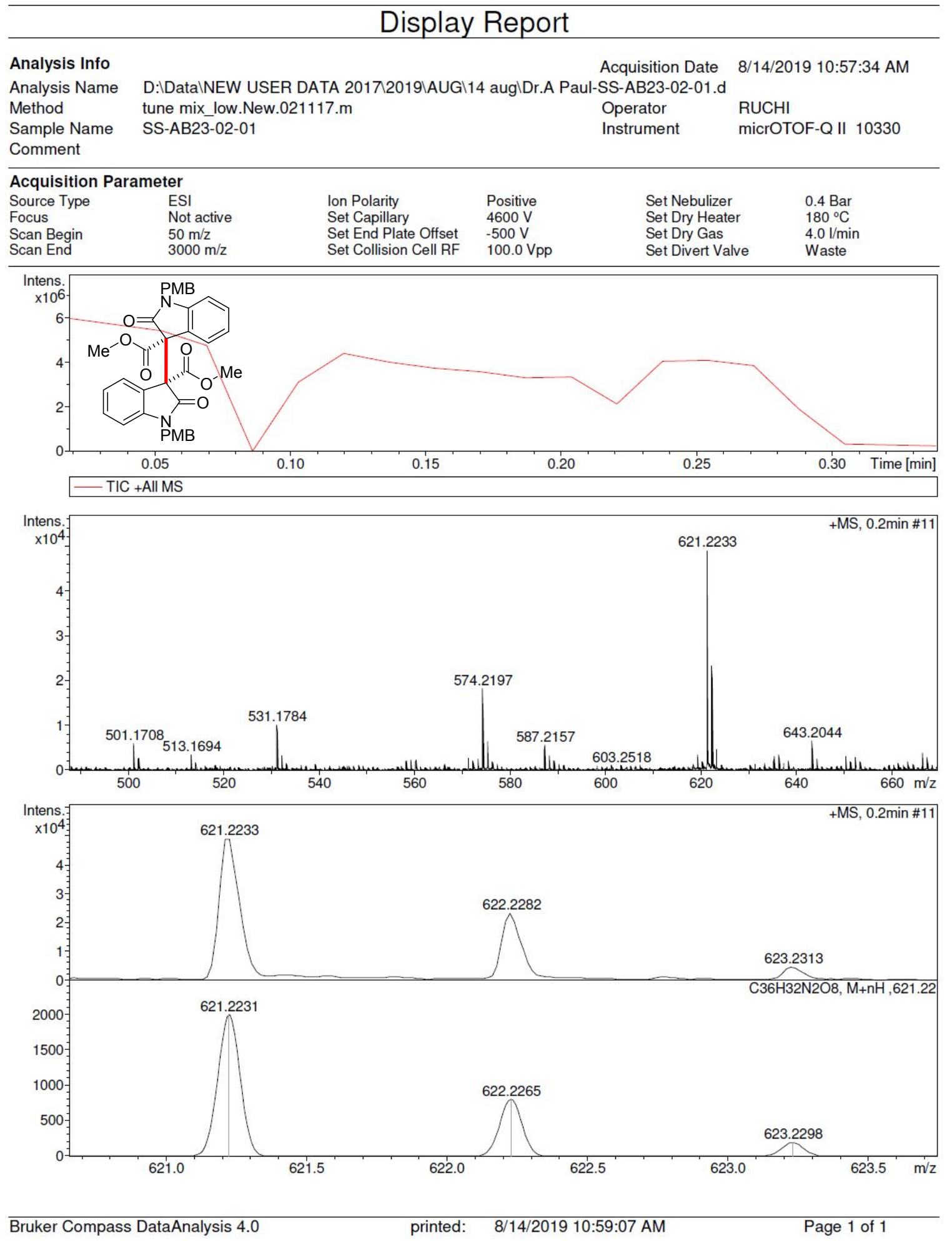

\section{Mass Spectrum of $\mathbf{4 d}$}




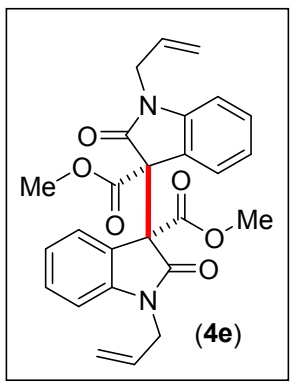

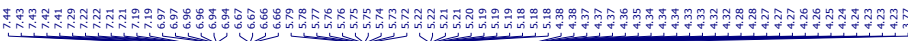
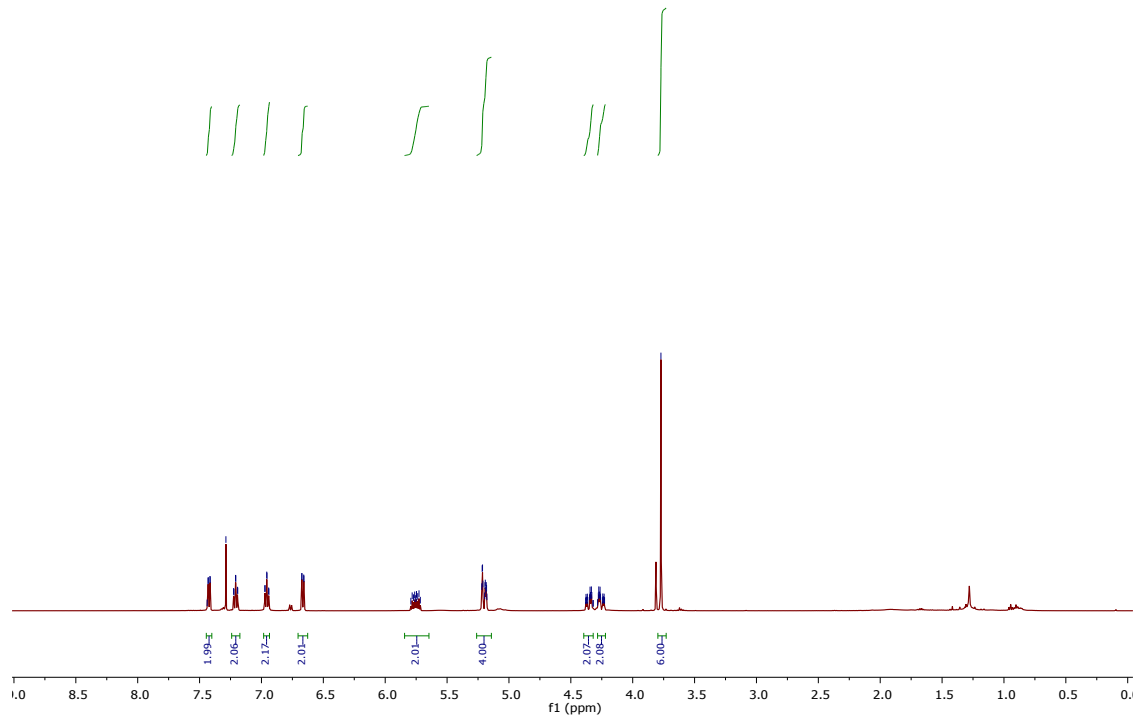

${ }^{1} \mathrm{H}$ NMR $\left(500 \mathrm{MHz}, \mathrm{CDCl}_{3}\right)$ of compound $4 \mathbf{e}$
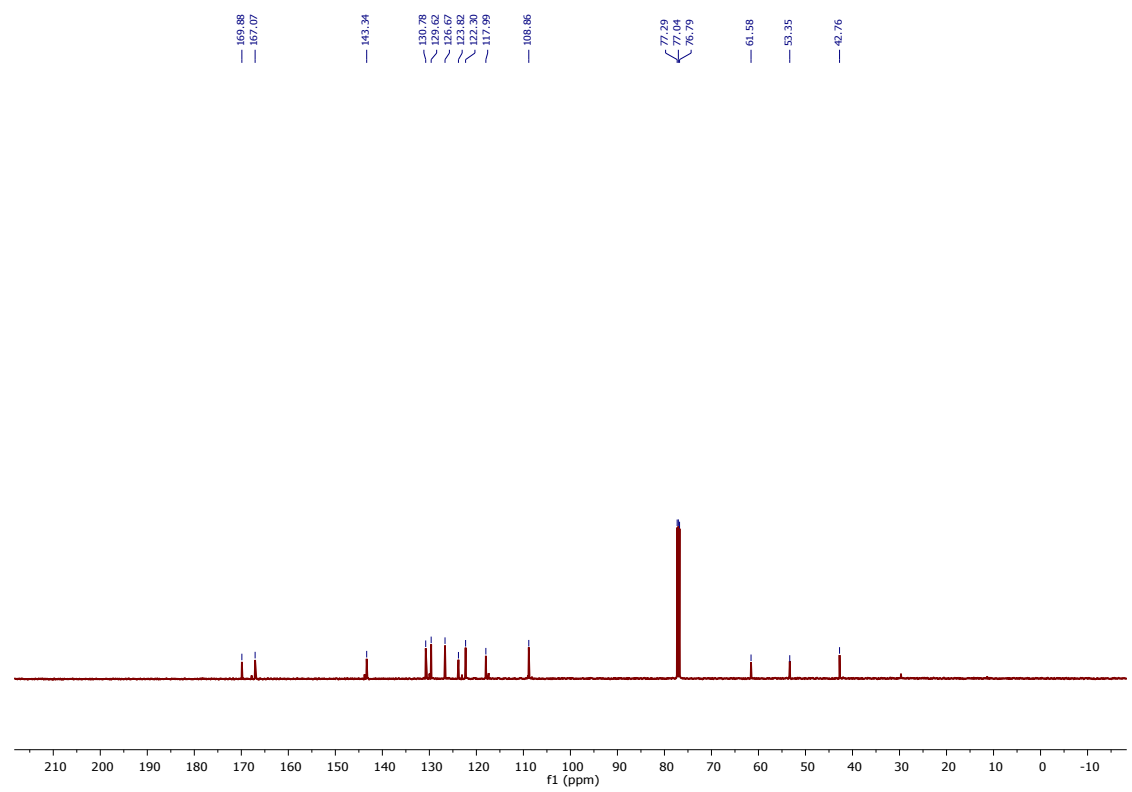

${ }^{13} \mathrm{C}\left\{{ }^{1} \mathrm{H}\right\}$ NMR $\left(125 \mathrm{MHz}, \mathrm{CDCl}_{3}\right)$ of compound $\mathbf{4 e}$ 


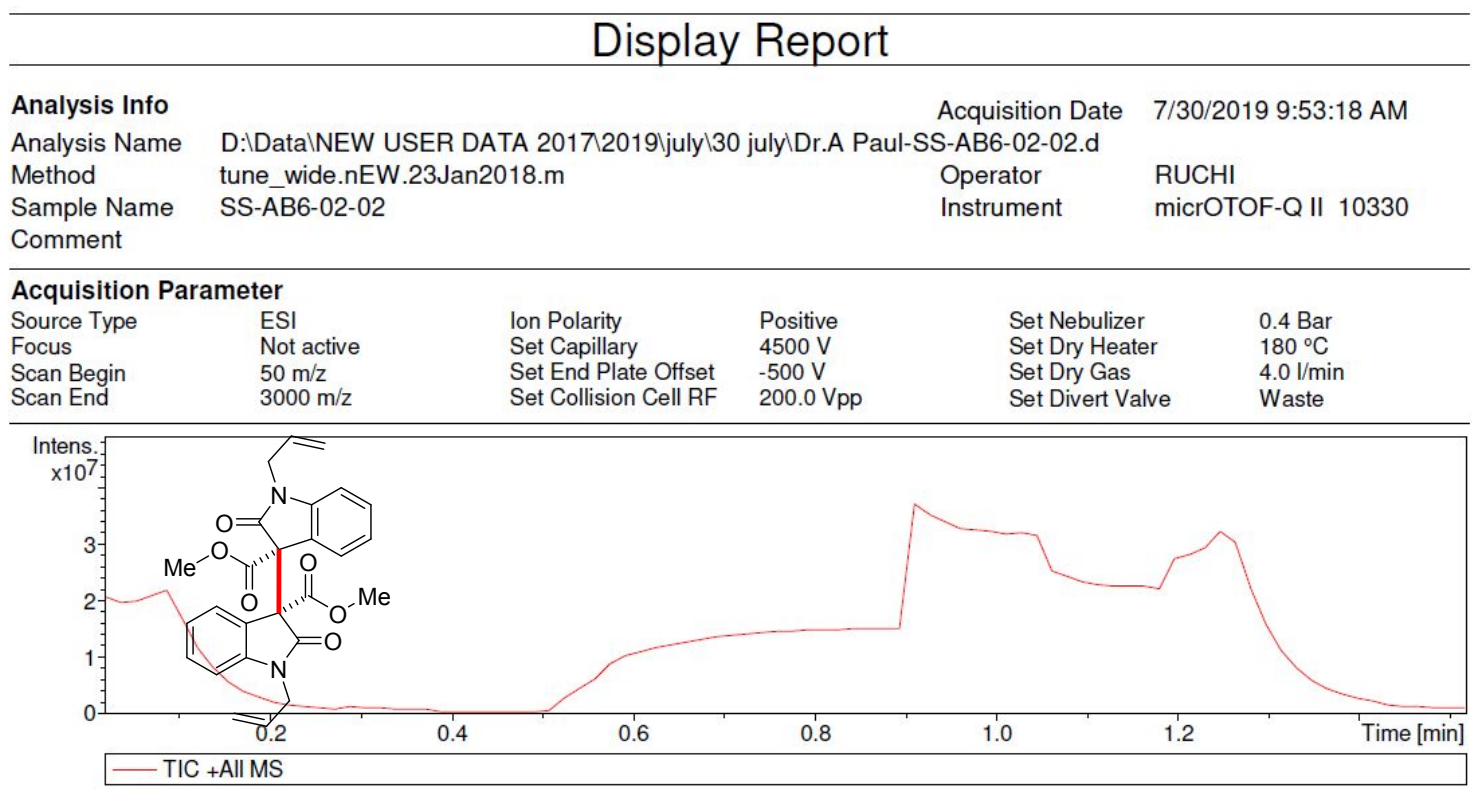
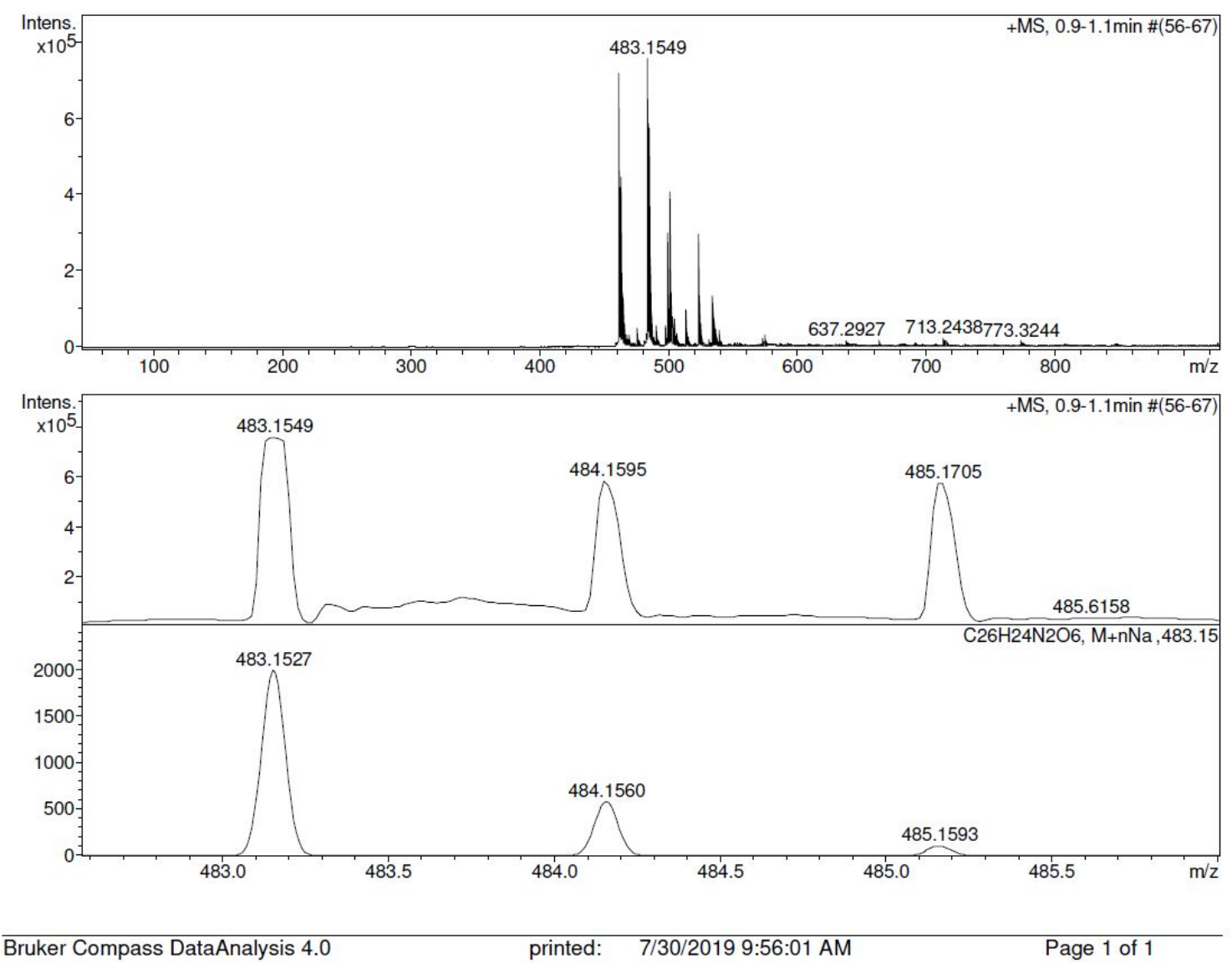

\section{Mass Spectrum of $\mathbf{4 e}$}




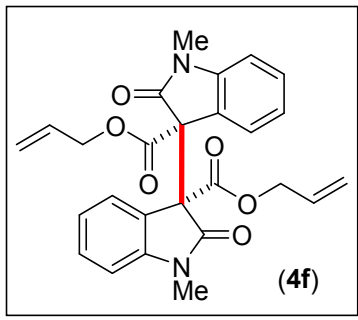

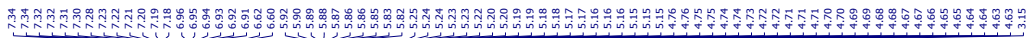

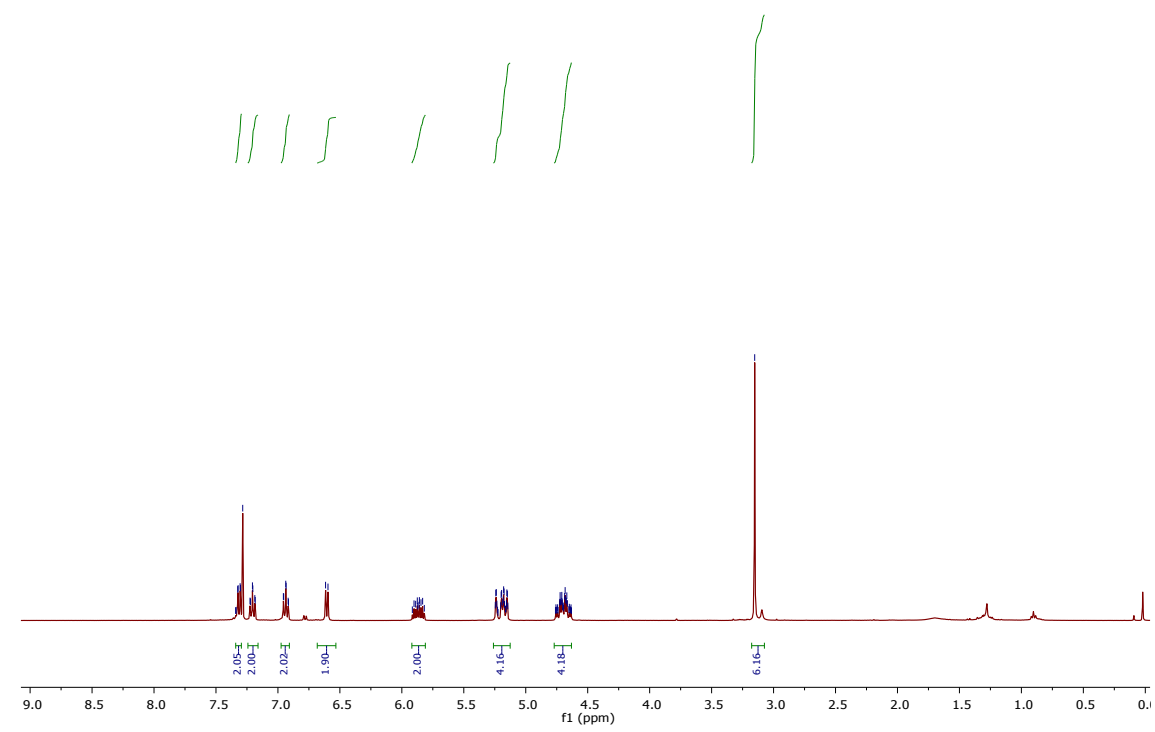

${ }^{1} \mathrm{H} \mathrm{NMR}\left(500 \mathrm{MHz}, \mathrm{CDCl}_{3}\right)$ of compound $\mathbf{4 f}$
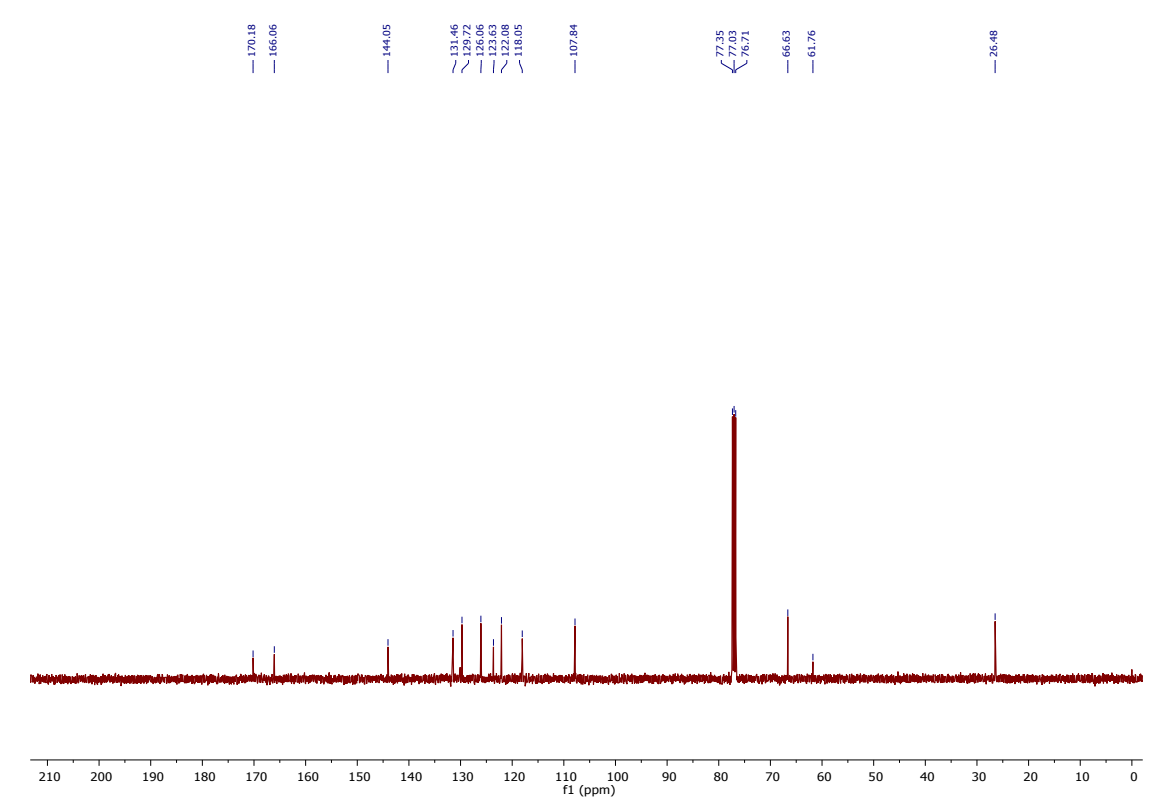

${ }^{13} \mathrm{C}\left\{{ }^{1} \mathrm{H}\right\} \mathrm{NMR}\left(125 \mathrm{MHz}, \mathrm{CDCl}_{3}\right)$ of compound $\mathbf{4 f}$ 


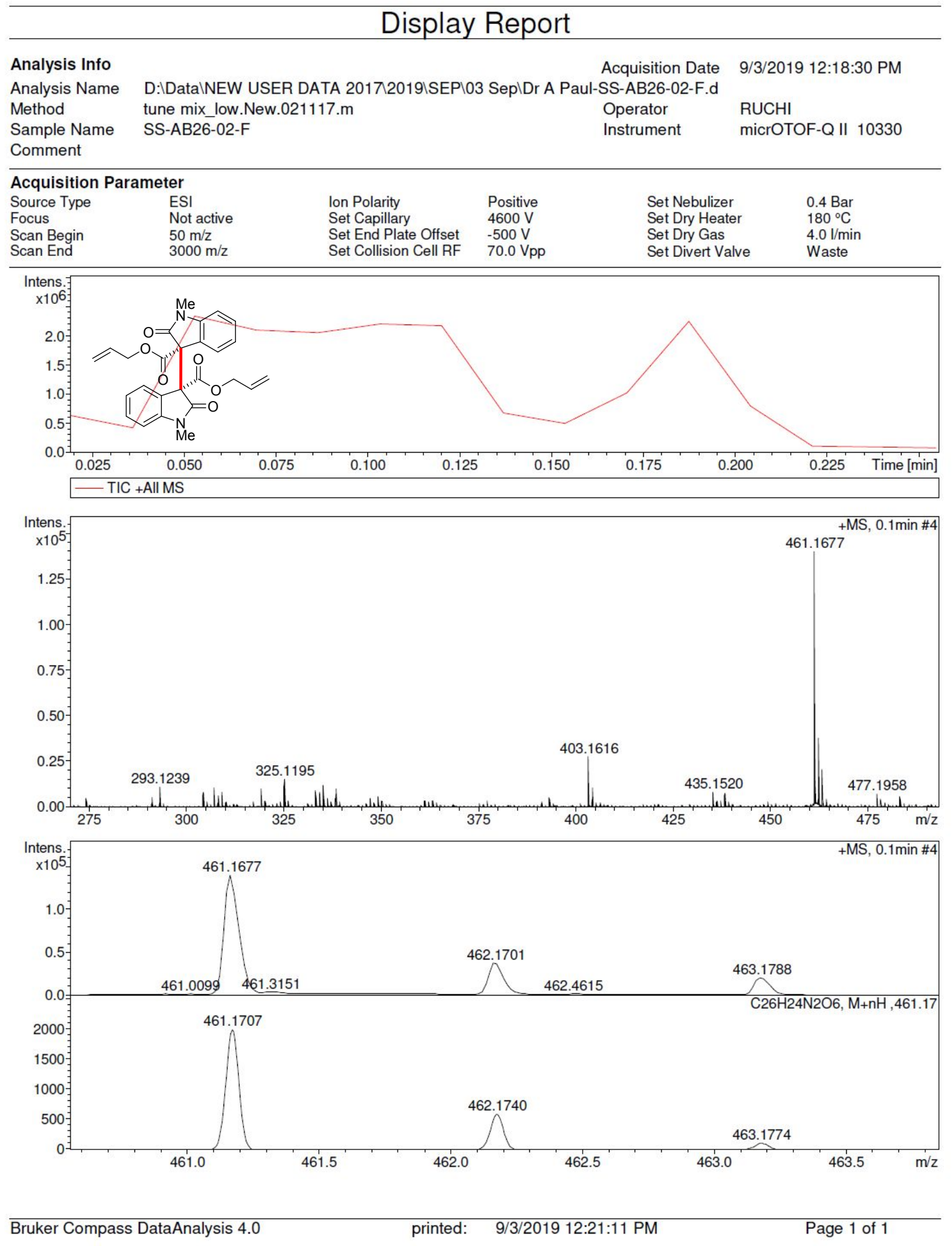

\section{Mass Spectrum of $\mathbf{4 f}$}




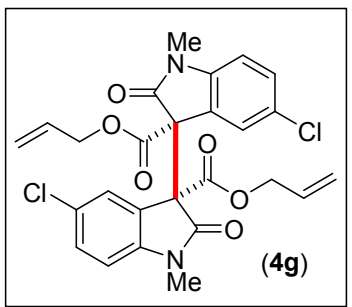

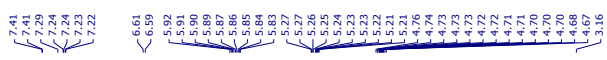
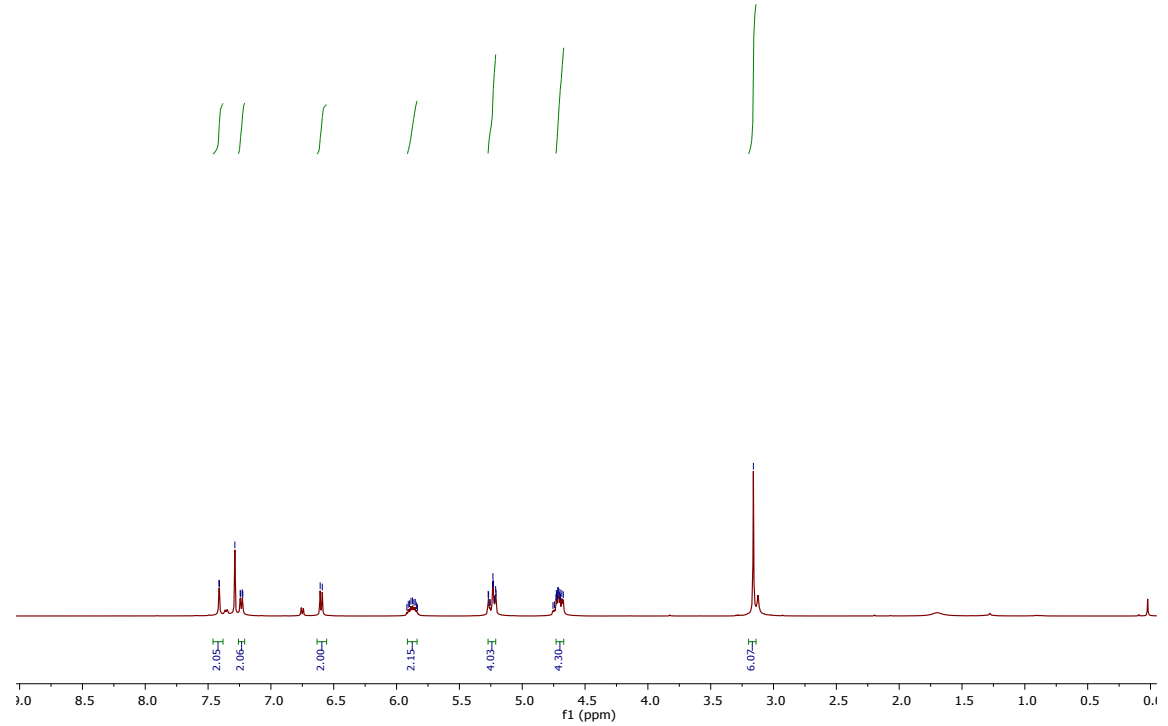

${ }^{1} \mathrm{H} \mathrm{NMR}\left(500 \mathrm{MHz}, \mathrm{CDCl}_{3}\right)$ of compound $\mathbf{4 g}$

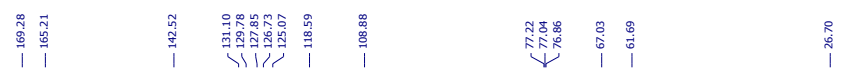

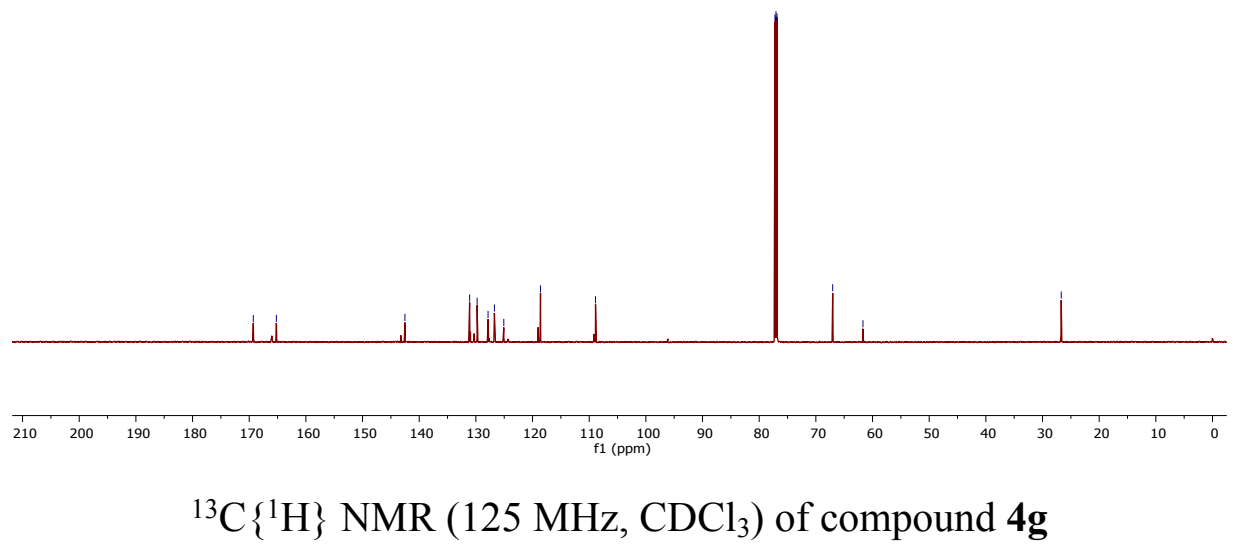




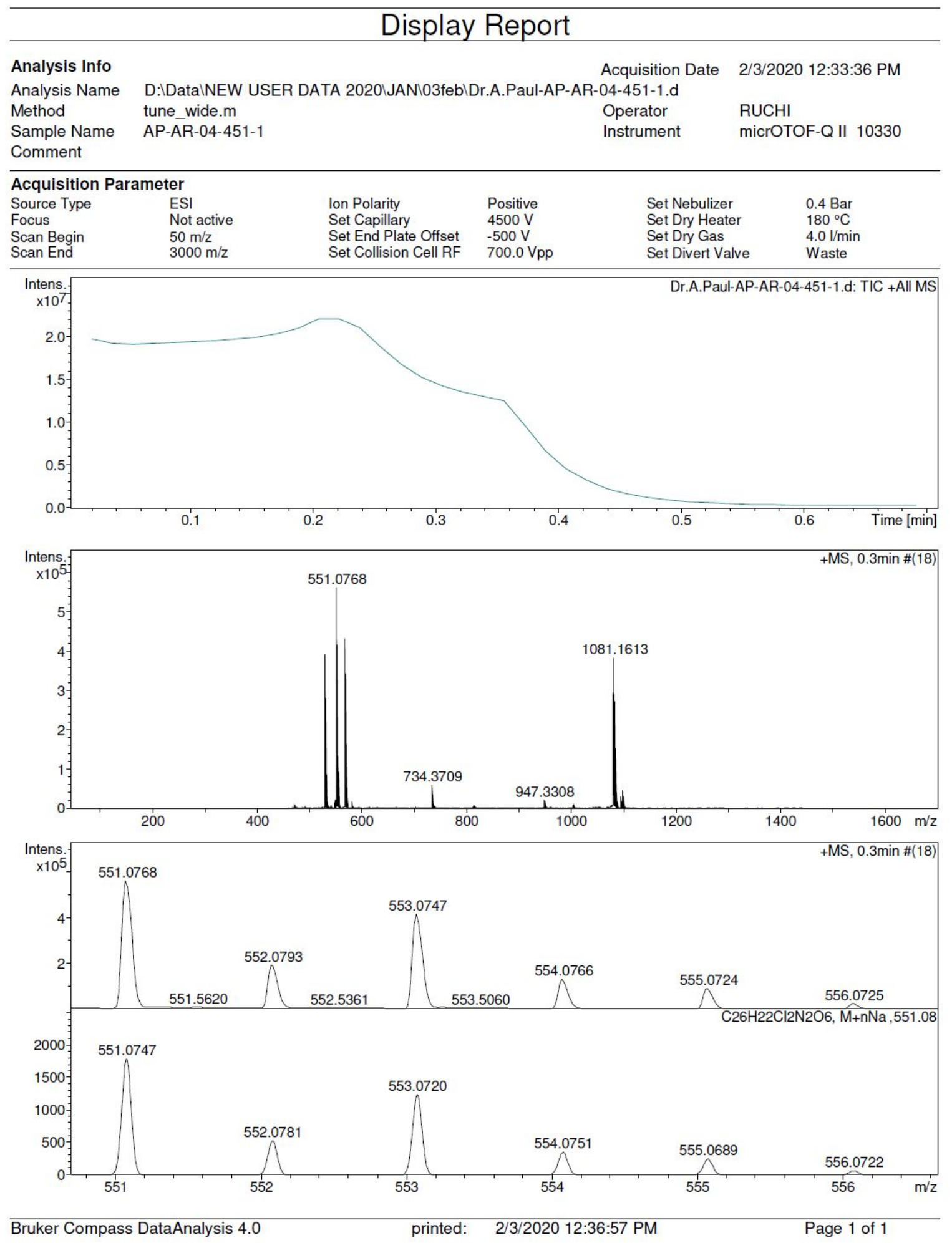

\section{Mass Spectrum of $\mathbf{4 g}$}



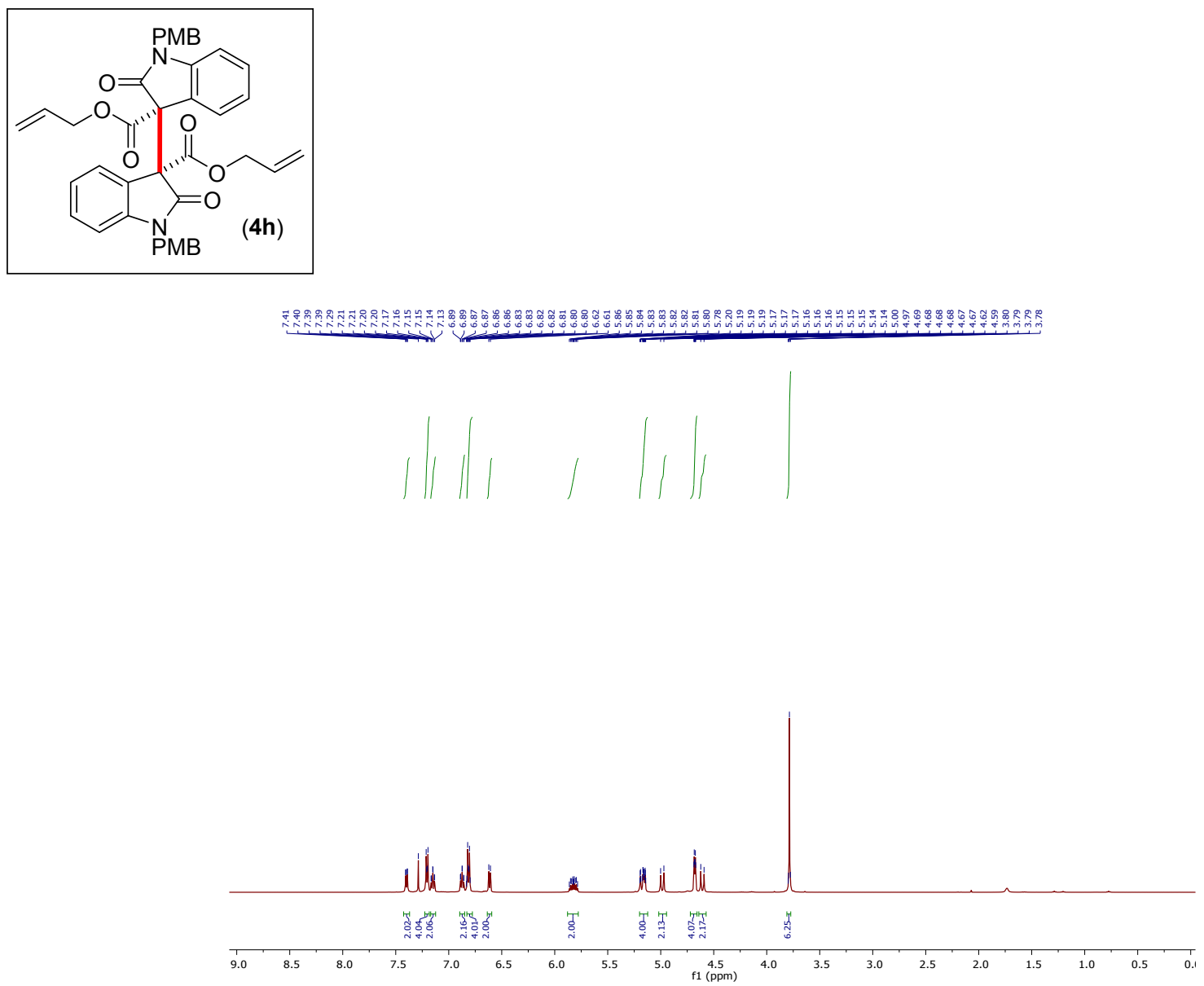

${ }^{1} \mathrm{H}$ NMR $\left(500 \mathrm{MHz}, \mathrm{CDCl}_{3}\right)$ of compound $\mathbf{4 h}$
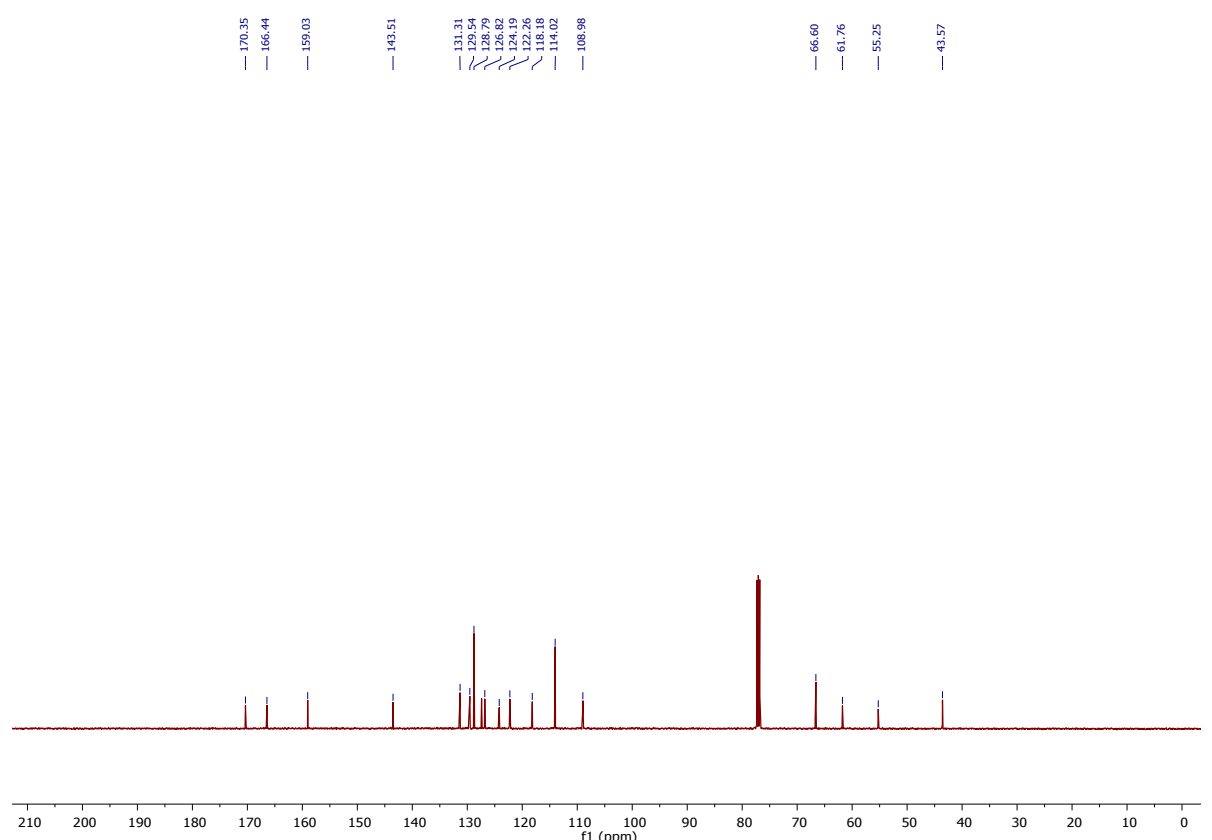

${ }^{13} \mathrm{C}\left\{{ }^{1} \mathrm{H}\right\}$ NMR (125 MHz, $\left.\mathrm{CDCl}_{3}\right)$ of compound $\mathbf{4 h}$ 


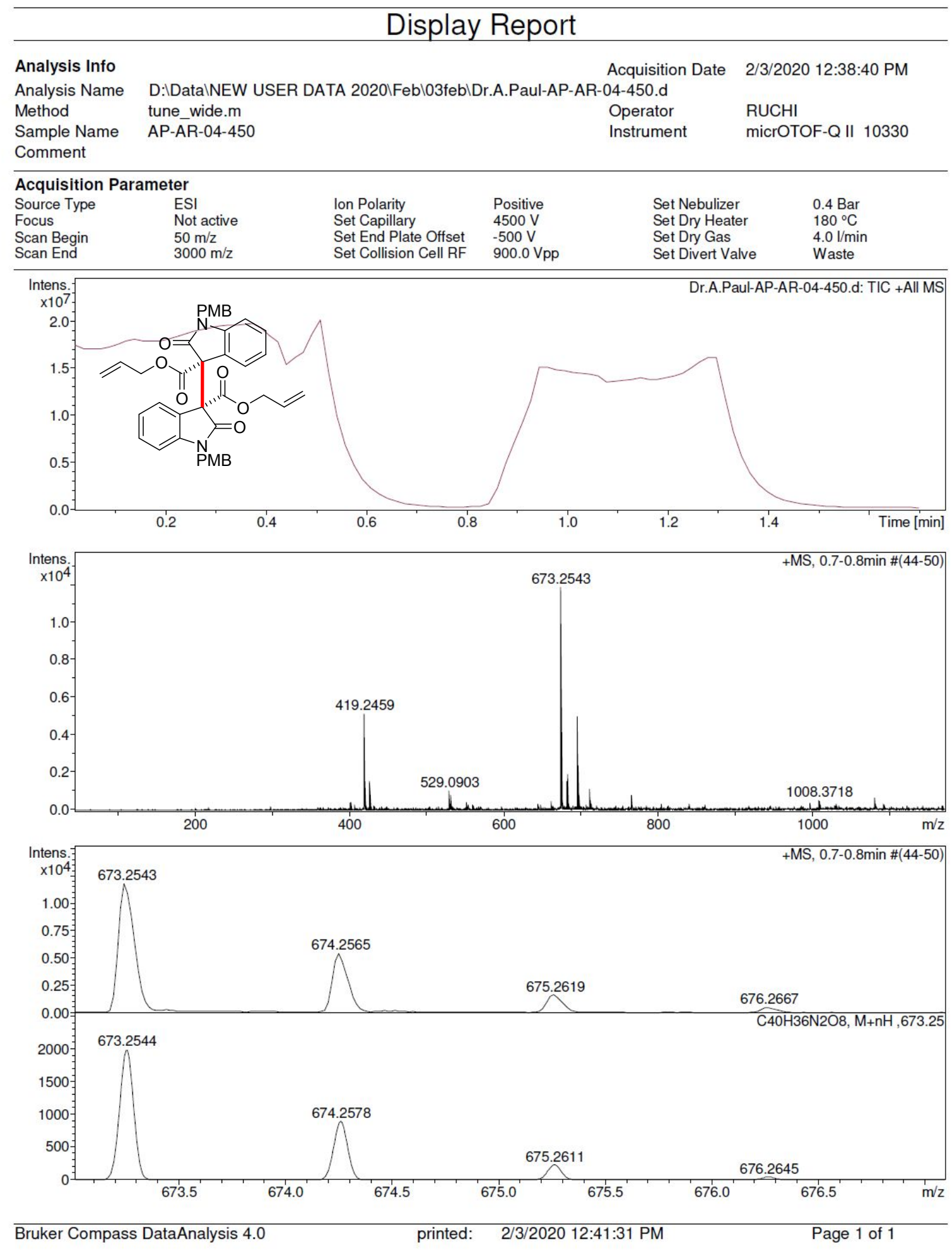

\section{Mass Spectrum of $\mathbf{4 h}$}




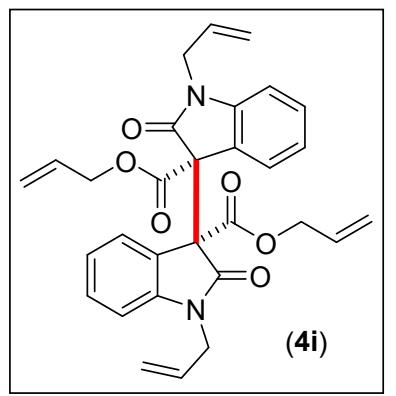

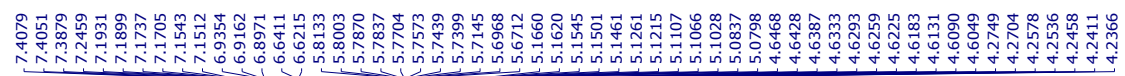
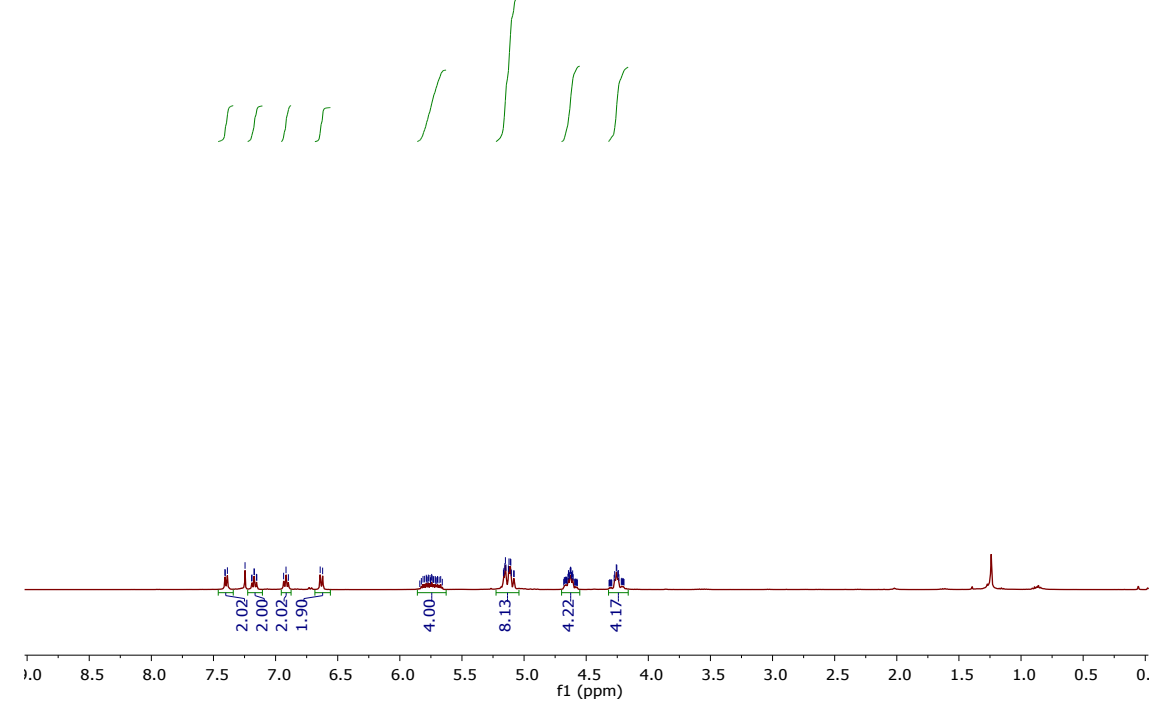

${ }^{1} \mathrm{H}$ NMR $\left(500 \mathrm{MHz}, \mathrm{CDCl}_{3}\right)$ of compound $4 \mathbf{i}$

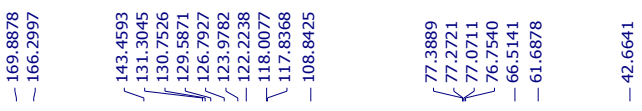

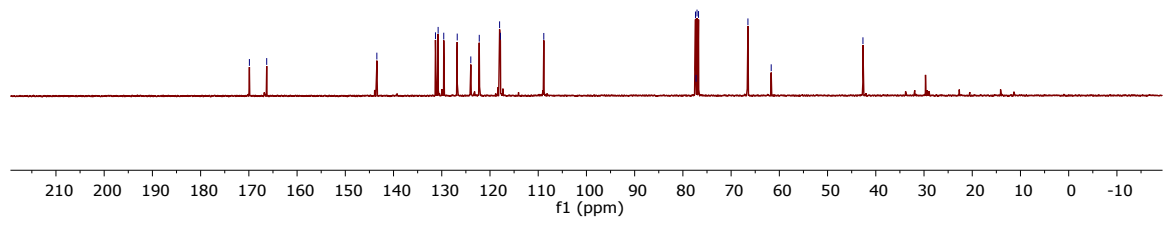

${ }^{13} \mathrm{C}\left\{{ }^{1} \mathrm{H}\right\}$ NMR $\left(125 \mathrm{MHz}, \mathrm{CDCl}_{3}\right)$ of compound $\mathbf{4 i}$ 


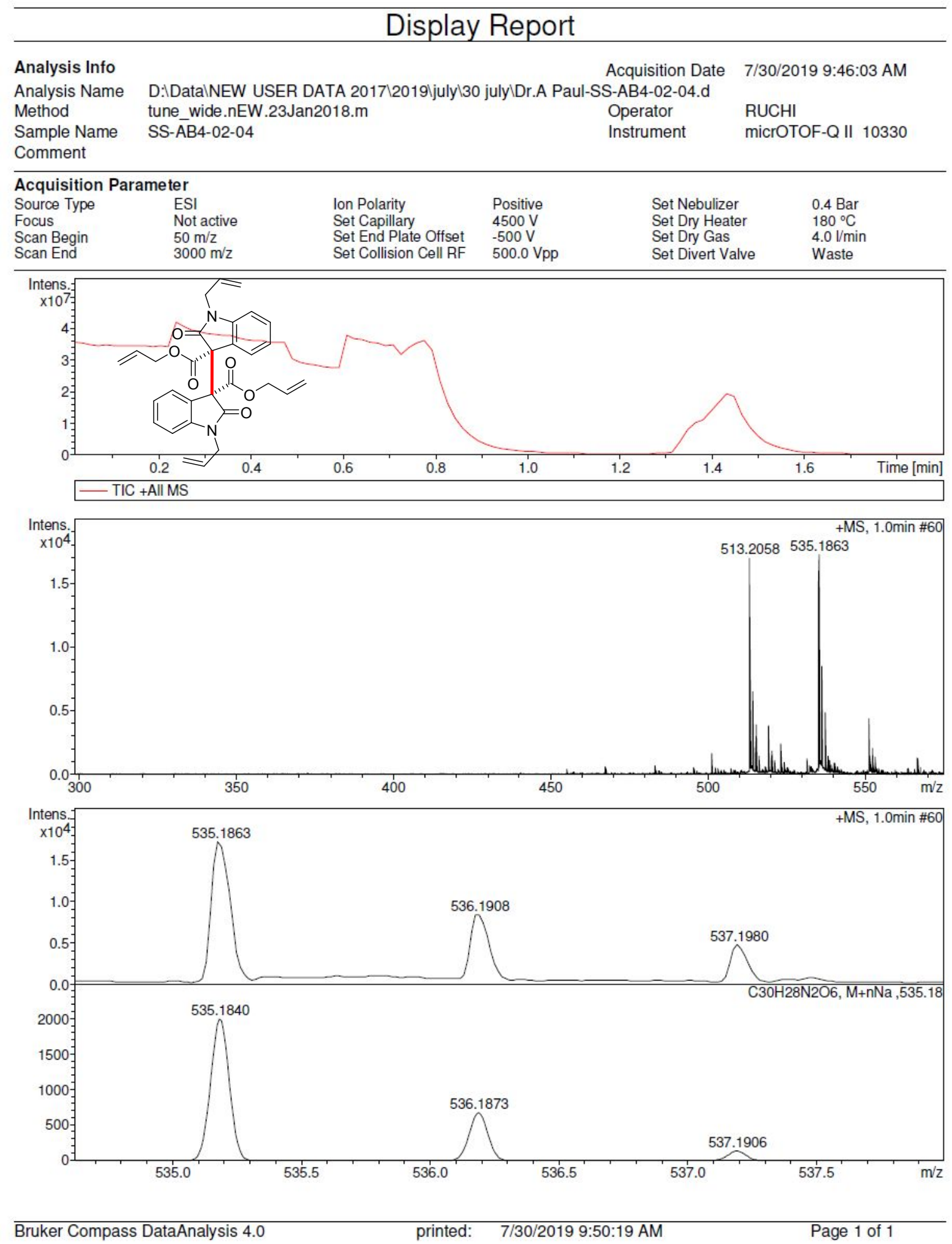

Mass Spectrum of $\mathbf{4 i}$ 

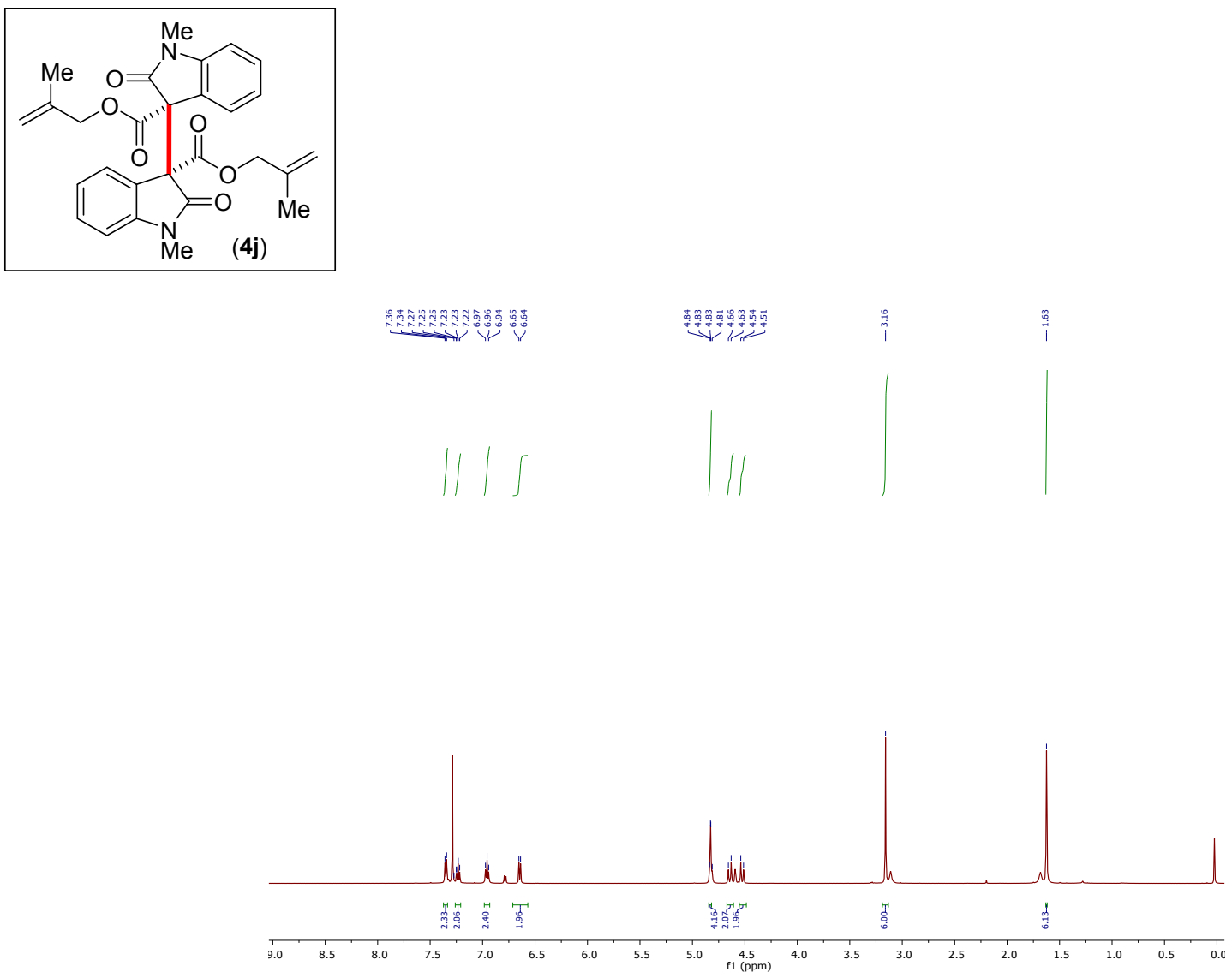

${ }^{1} \mathrm{H}$ NMR (500 MHz, $\mathrm{CDCl}_{3}$ ) of compound $\mathbf{4 j}$
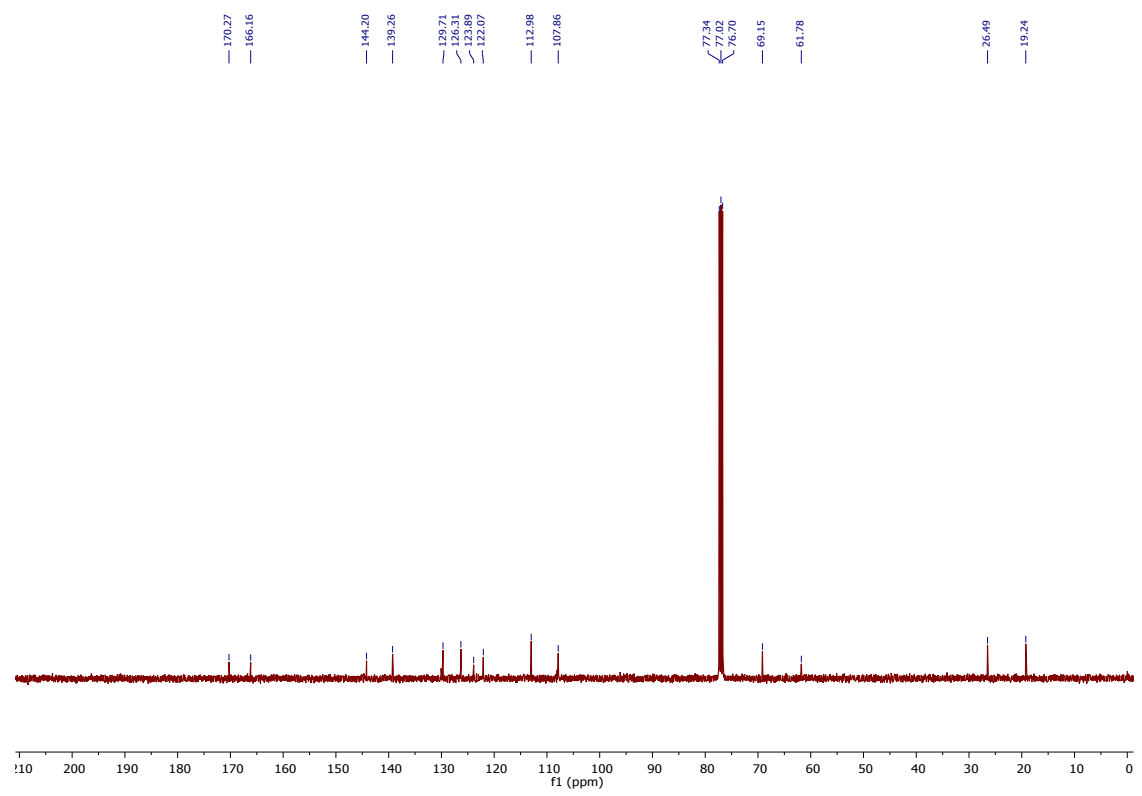

${ }^{13} \mathrm{C}\left\{{ }^{1} \mathrm{H}\right\} \mathrm{NMR}\left(125 \mathrm{MHz}, \mathrm{CDCl}_{3}\right)$ of compound $\mathbf{4 j}$ 


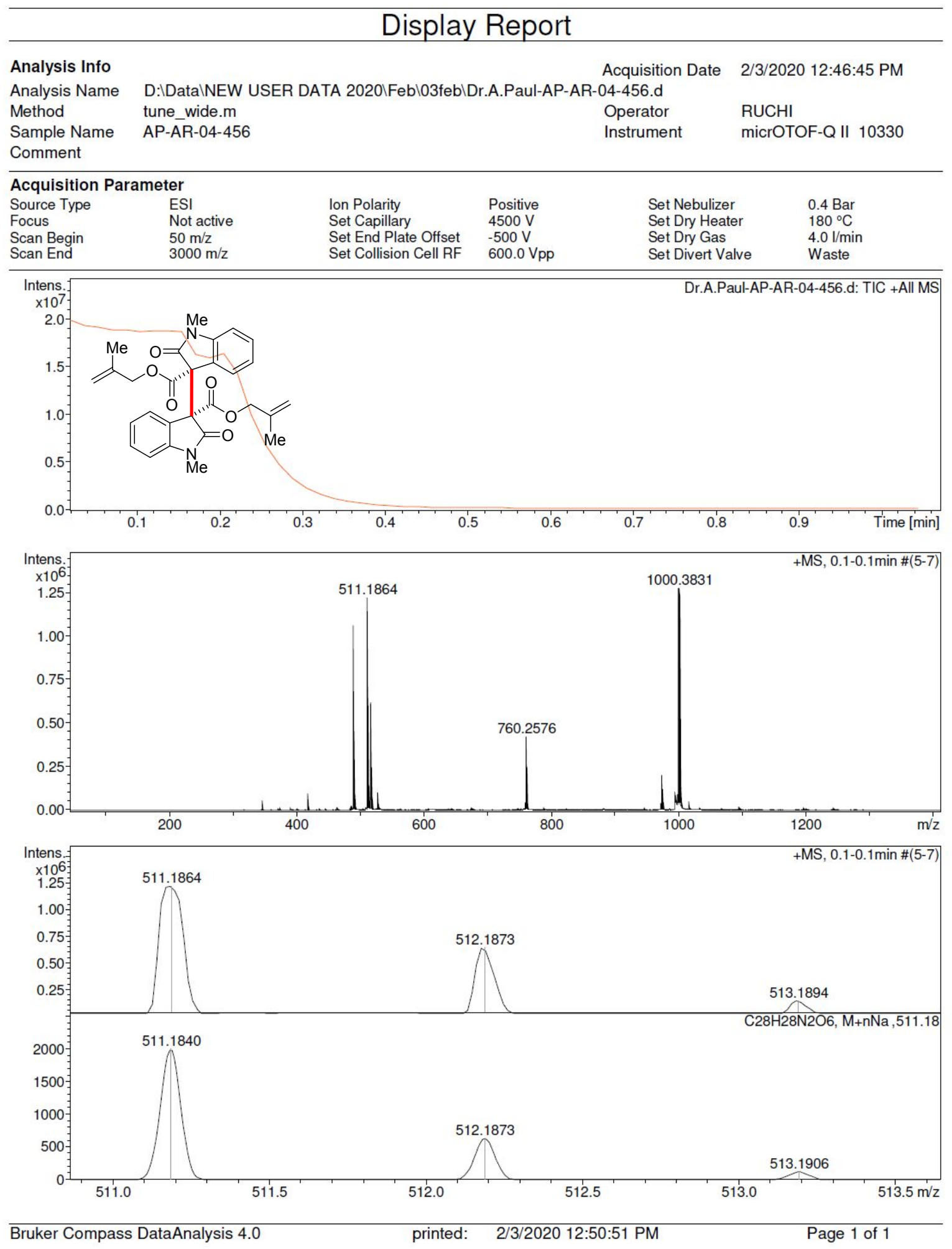

\section{Mass Spectrum of $\mathbf{4} \mathbf{j}$}



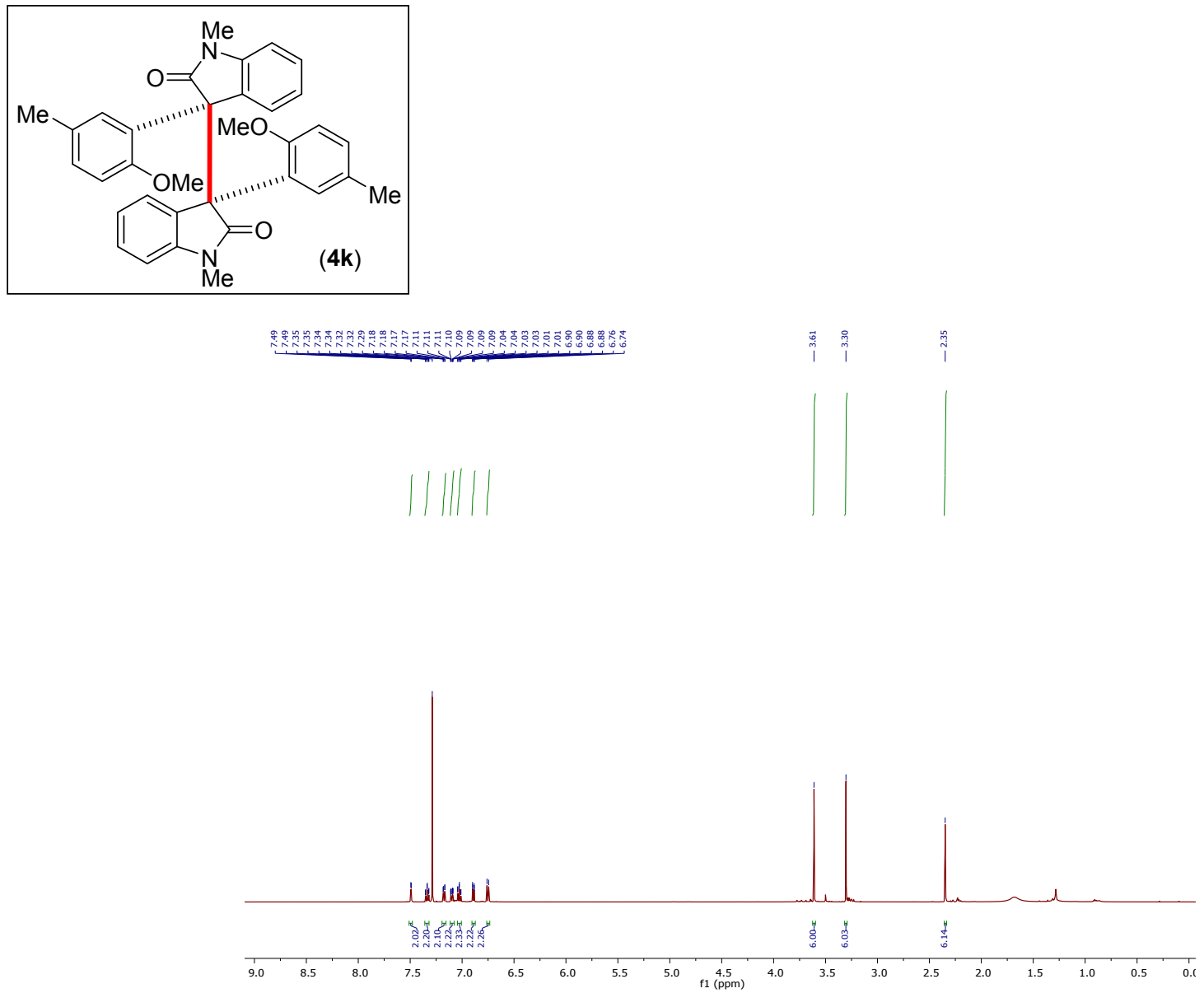

${ }^{1} \mathrm{H}$ NMR (500 MHz, $\mathrm{CDCl}_{3}$ ) of compound $4 \mathbf{k}$

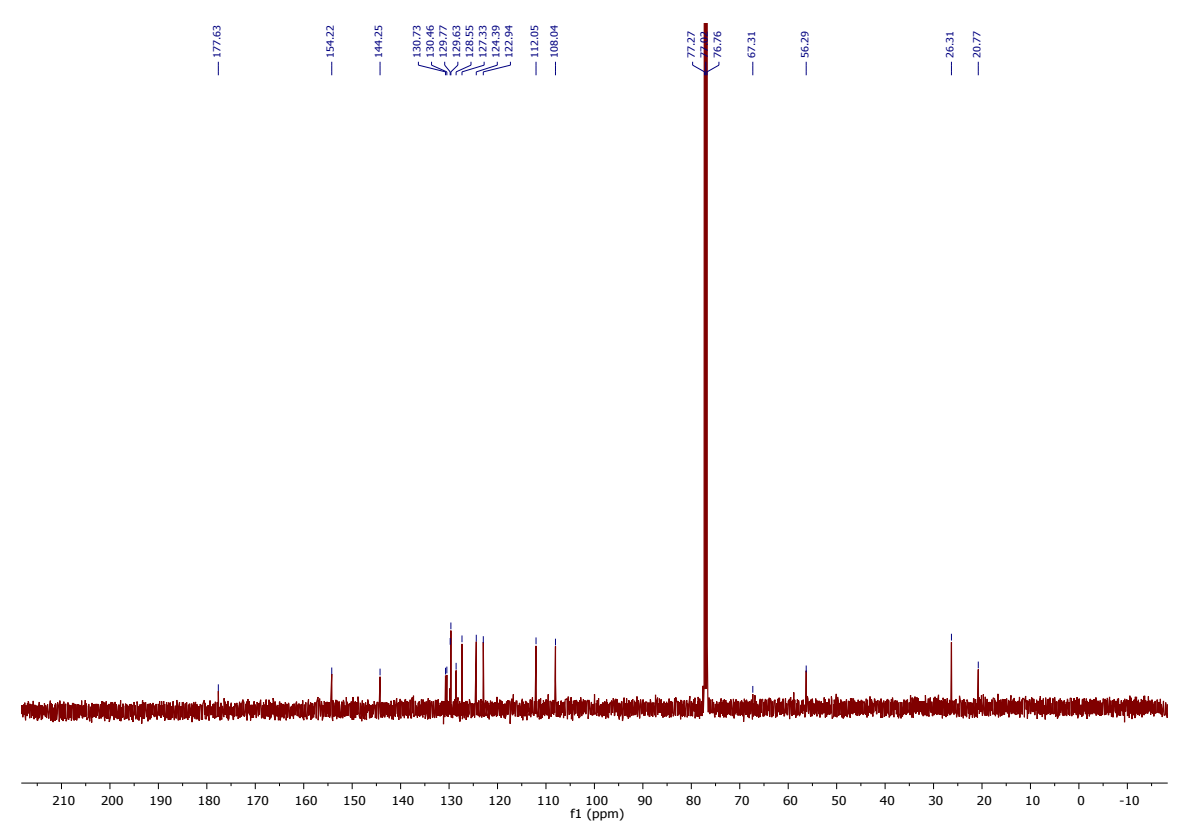

${ }^{13} \mathrm{C}\left\{{ }^{1} \mathrm{H}\right\}$ NMR $\left(125 \mathrm{MHz}, \mathrm{CDCl}_{3}\right)$ of compound $\mathbf{4 k}$ 


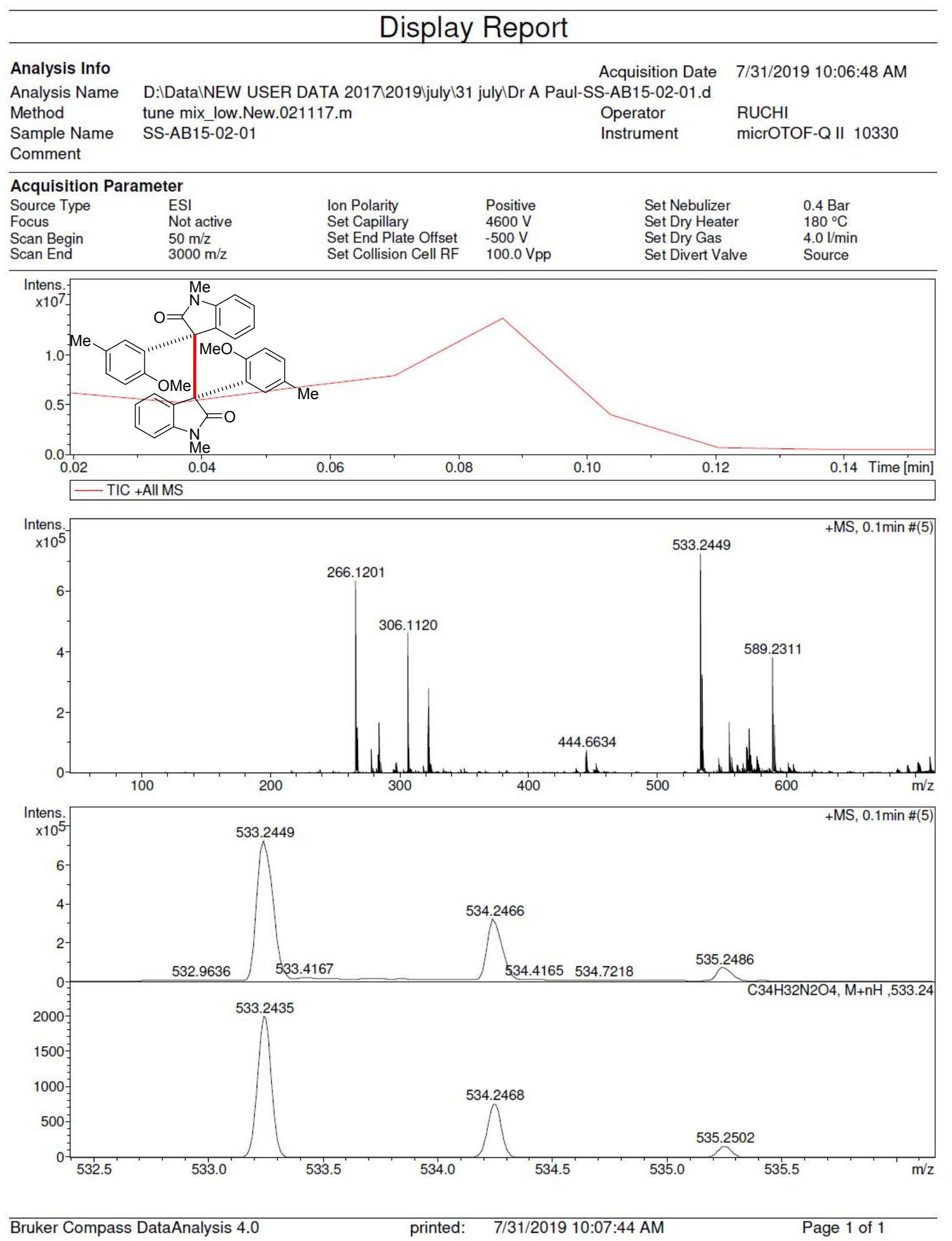

\section{Mass Spectrum of $\mathbf{4 k}$}




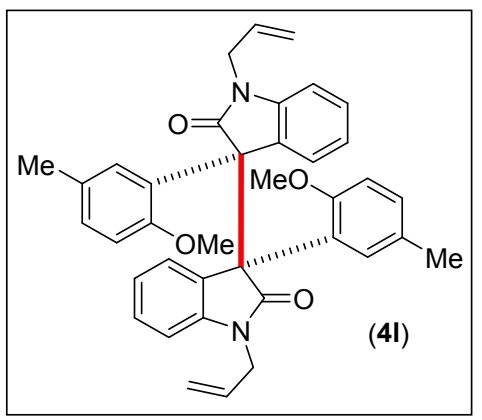

克琞

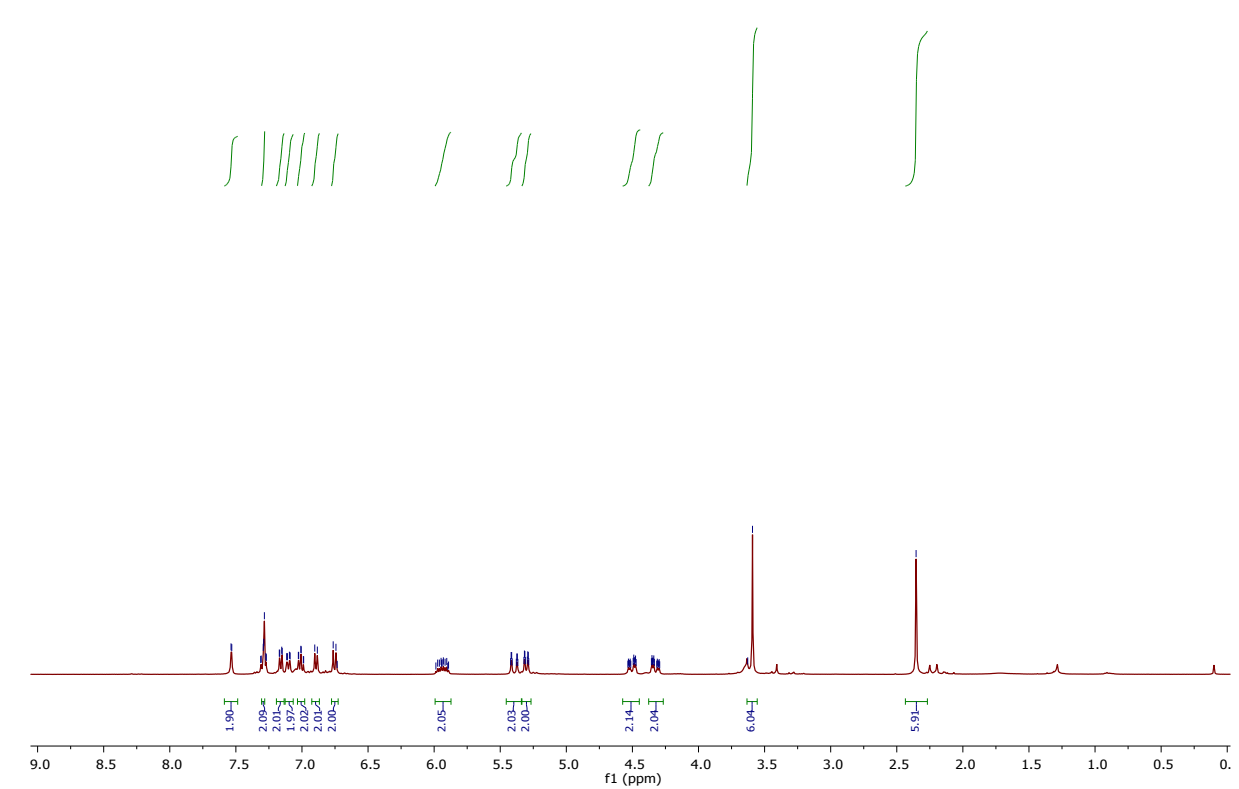

${ }^{1} \mathrm{H}$ NMR (400 MHz, $\left.\mathrm{CDCl}_{3}\right)$ of compound $4 \mathbf{l}$

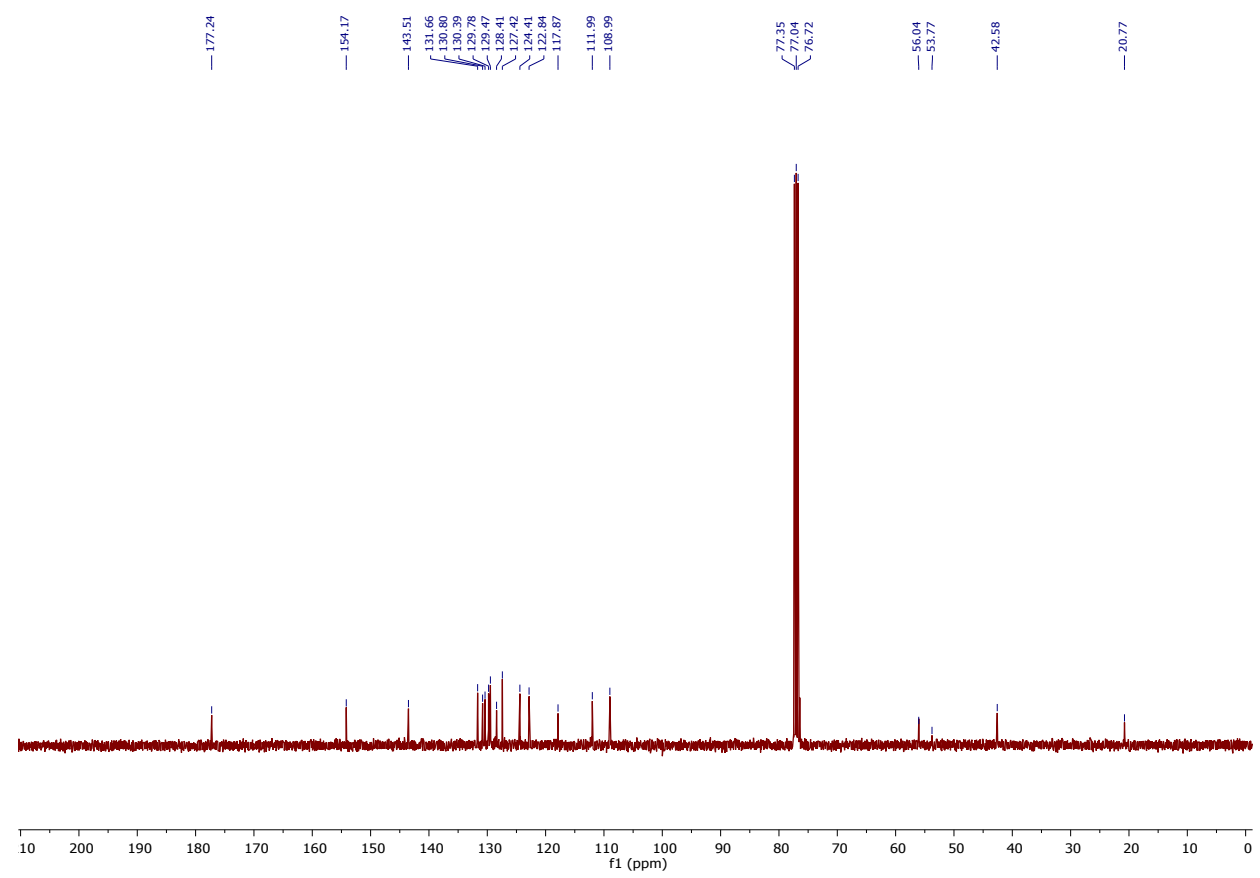

${ }^{13} \mathrm{C}\left\{{ }^{1} \mathrm{H}\right\} \mathrm{NMR}\left(100 \mathrm{MHz}, \mathrm{CDCl}_{3}\right)$ of compound $4 \mathbf{l}$ 


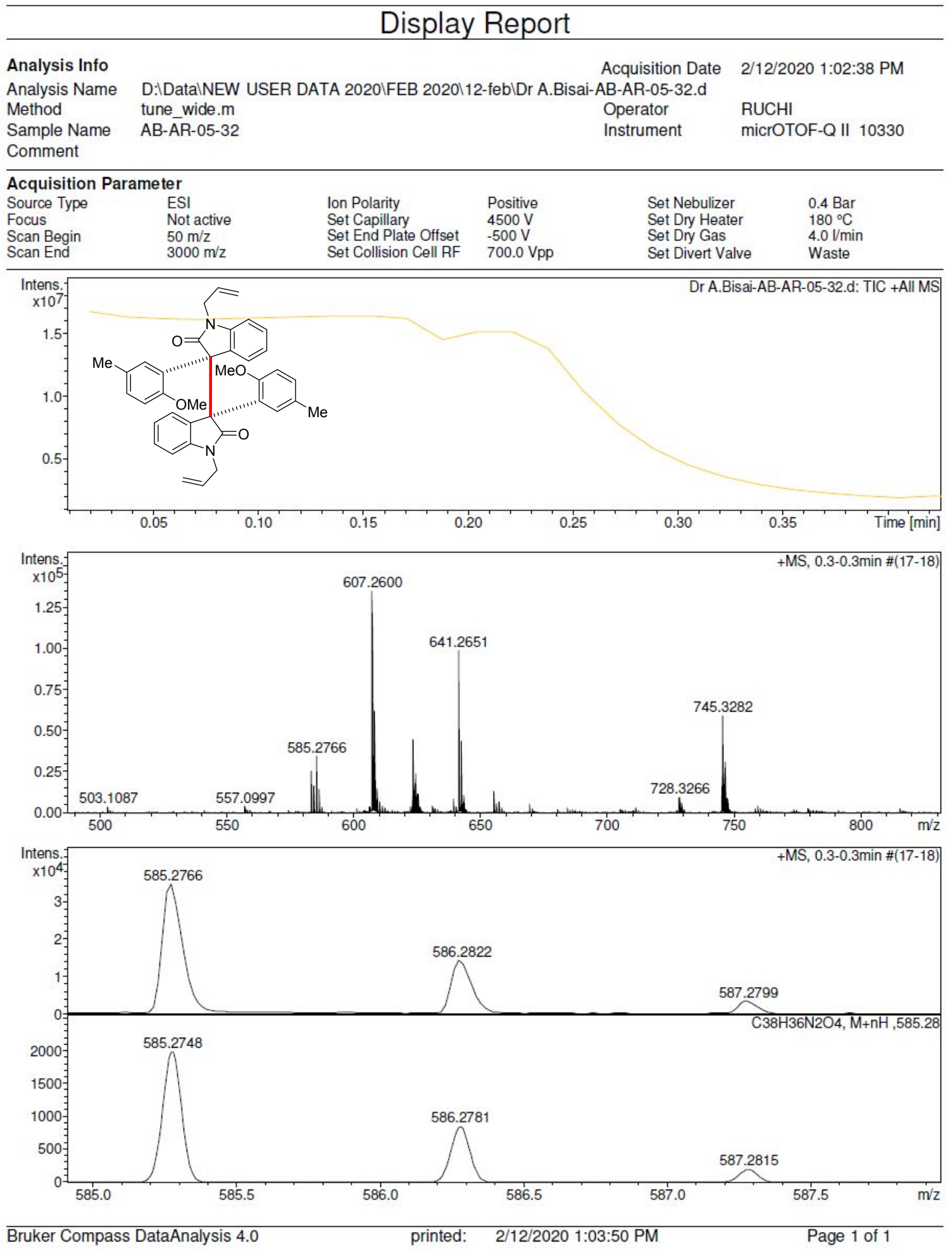

\section{Mass Spectrum of 4l}




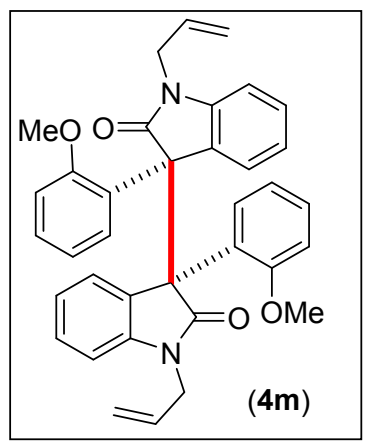

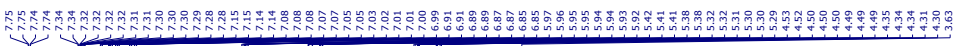
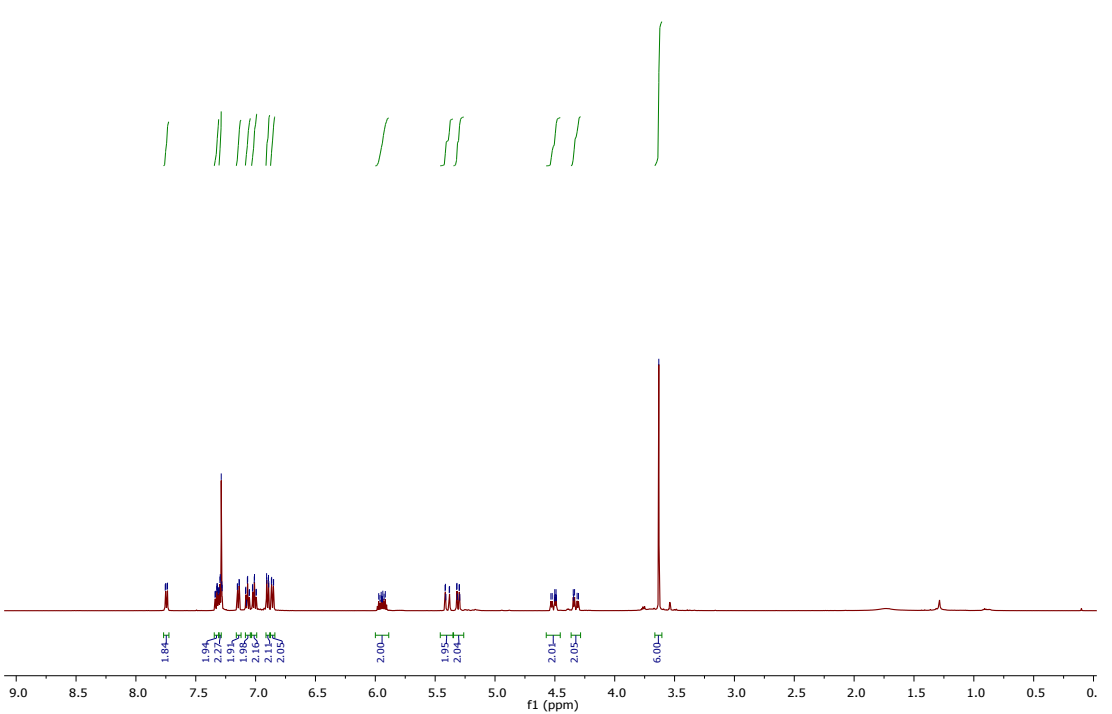

${ }^{1} \mathrm{H} \mathrm{NMR}\left(500 \mathrm{MHz}, \mathrm{CDCl}_{3}\right)$ of compound-4m
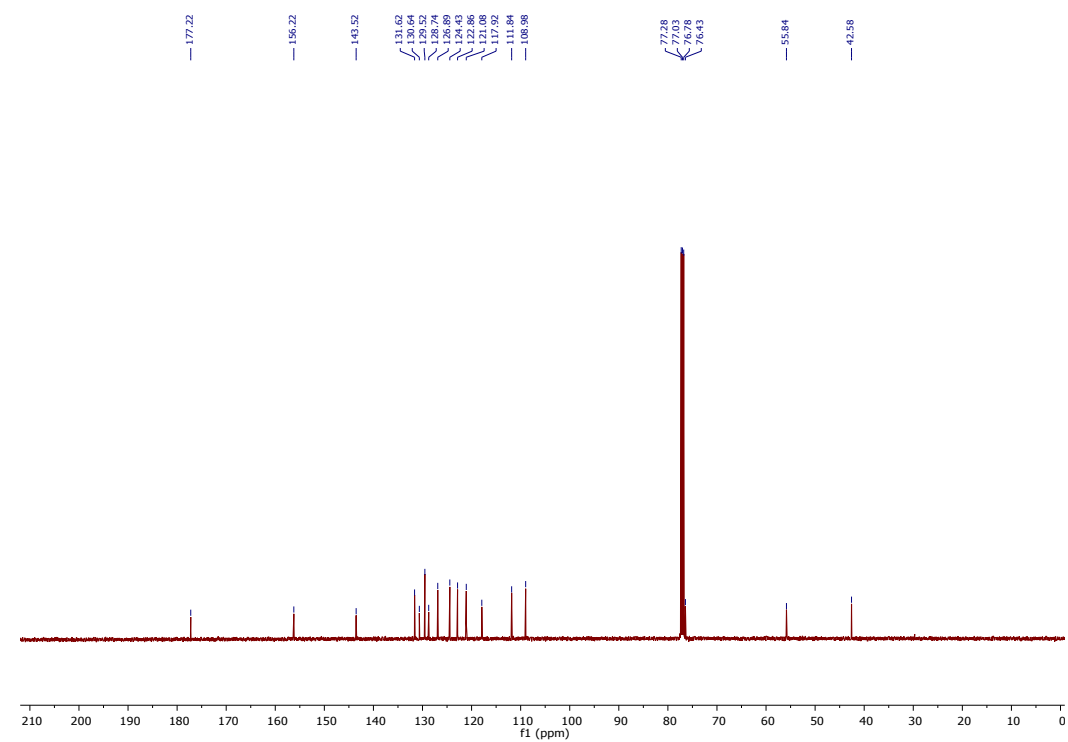

${ }^{13} \mathrm{C}\left\{{ }^{1} \mathrm{H}\right\}$ NMR $\left(125 \mathrm{MHz}, \mathrm{CDCl}_{3}\right)$ of compound $\mathbf{4 m}$ 


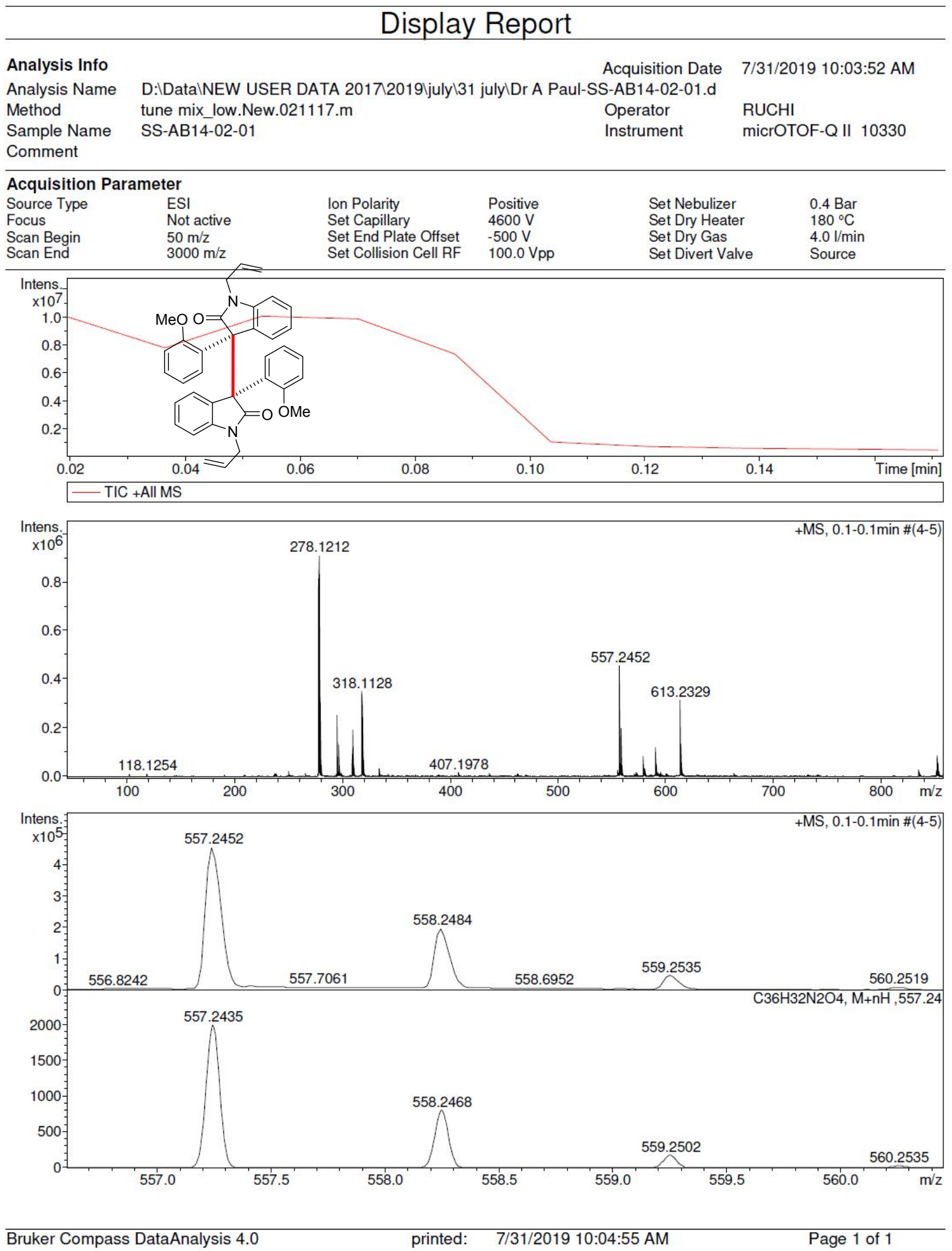

Mass Spectrum of $\mathbf{4 m}$ 

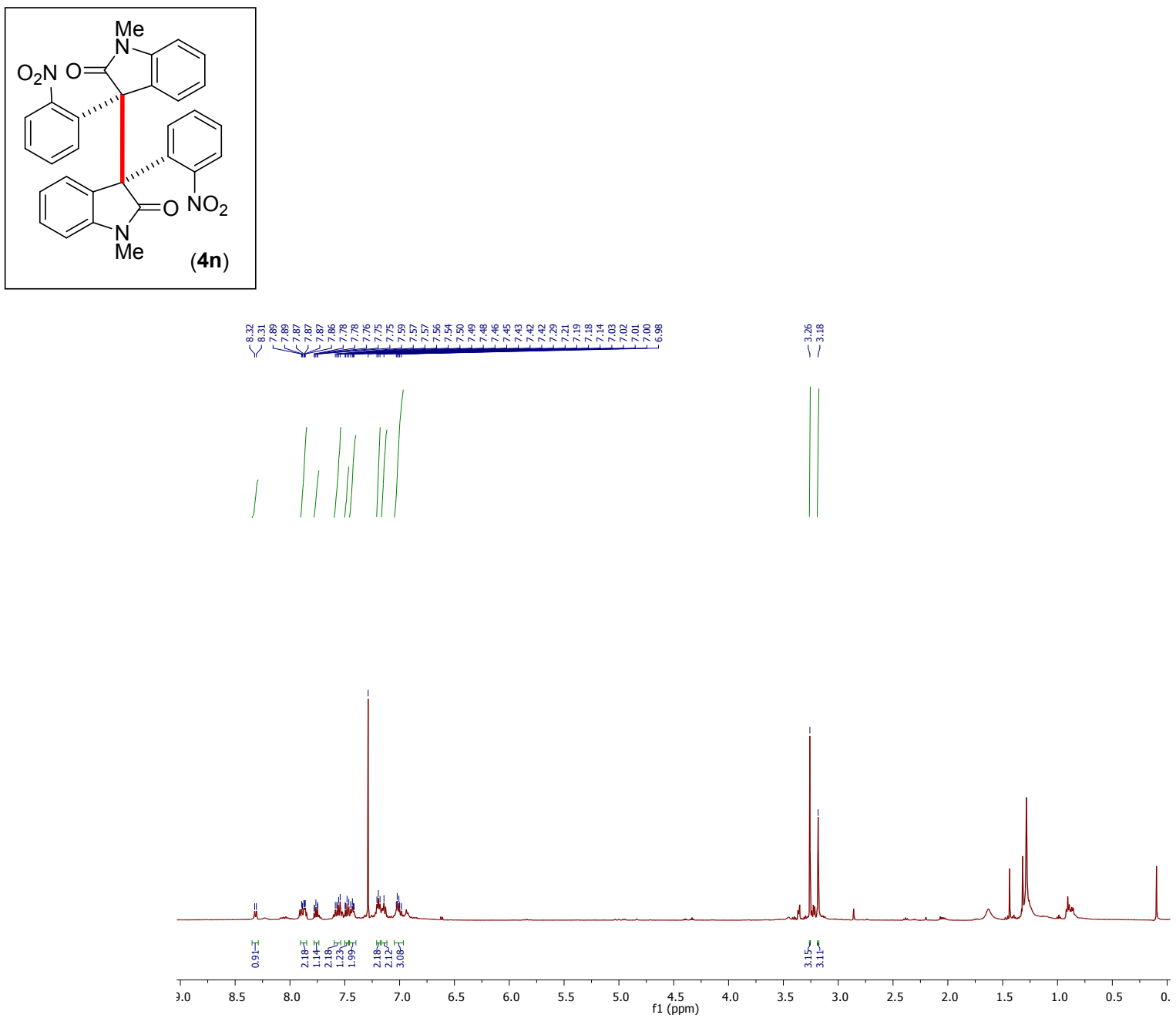

${ }^{1} \mathrm{H}$ NMR $\left(500 \mathrm{MHz}, \mathrm{CDCl}_{3}\right)$ of compound $\mathbf{4 n}$

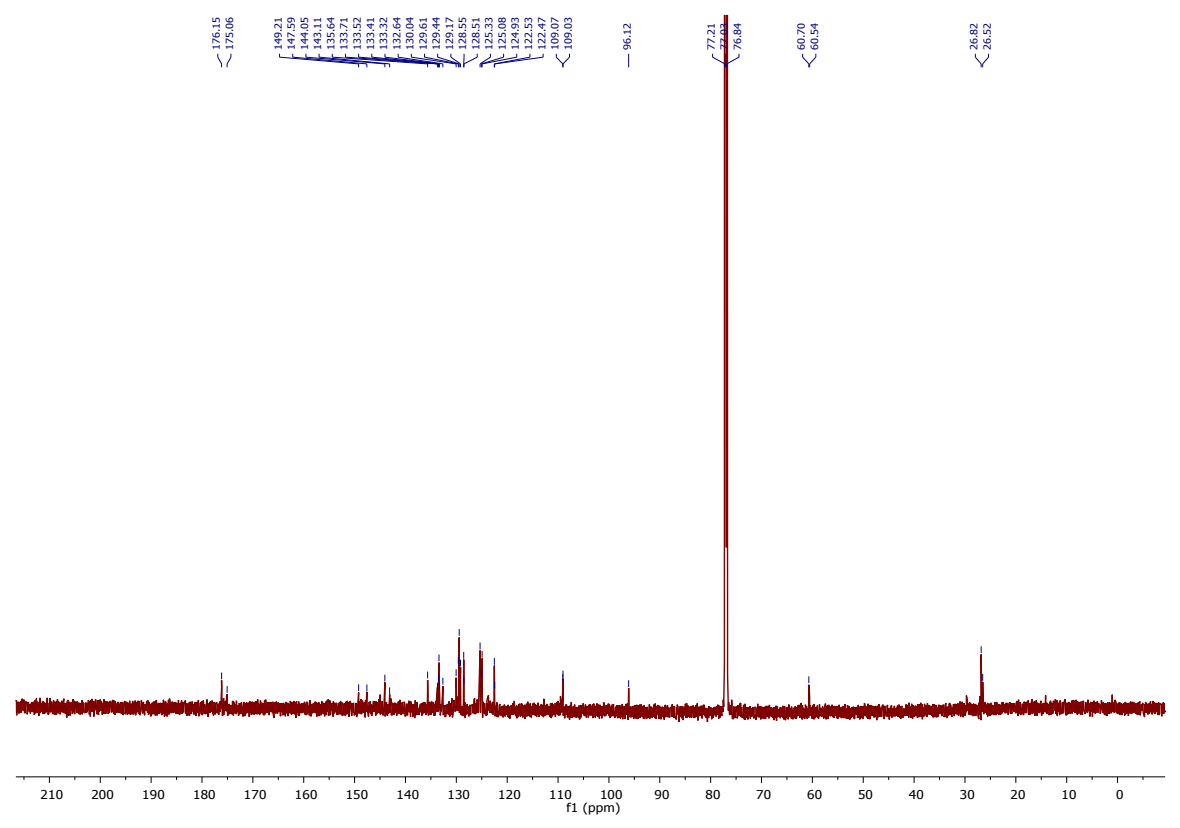

${ }^{13} \mathrm{C}\left\{{ }^{1} \mathrm{H}\right\}$ NMR (125 MHz, $\left.\mathrm{CDCl}_{3}\right)$ of compound $\mathbf{4 n}$ 


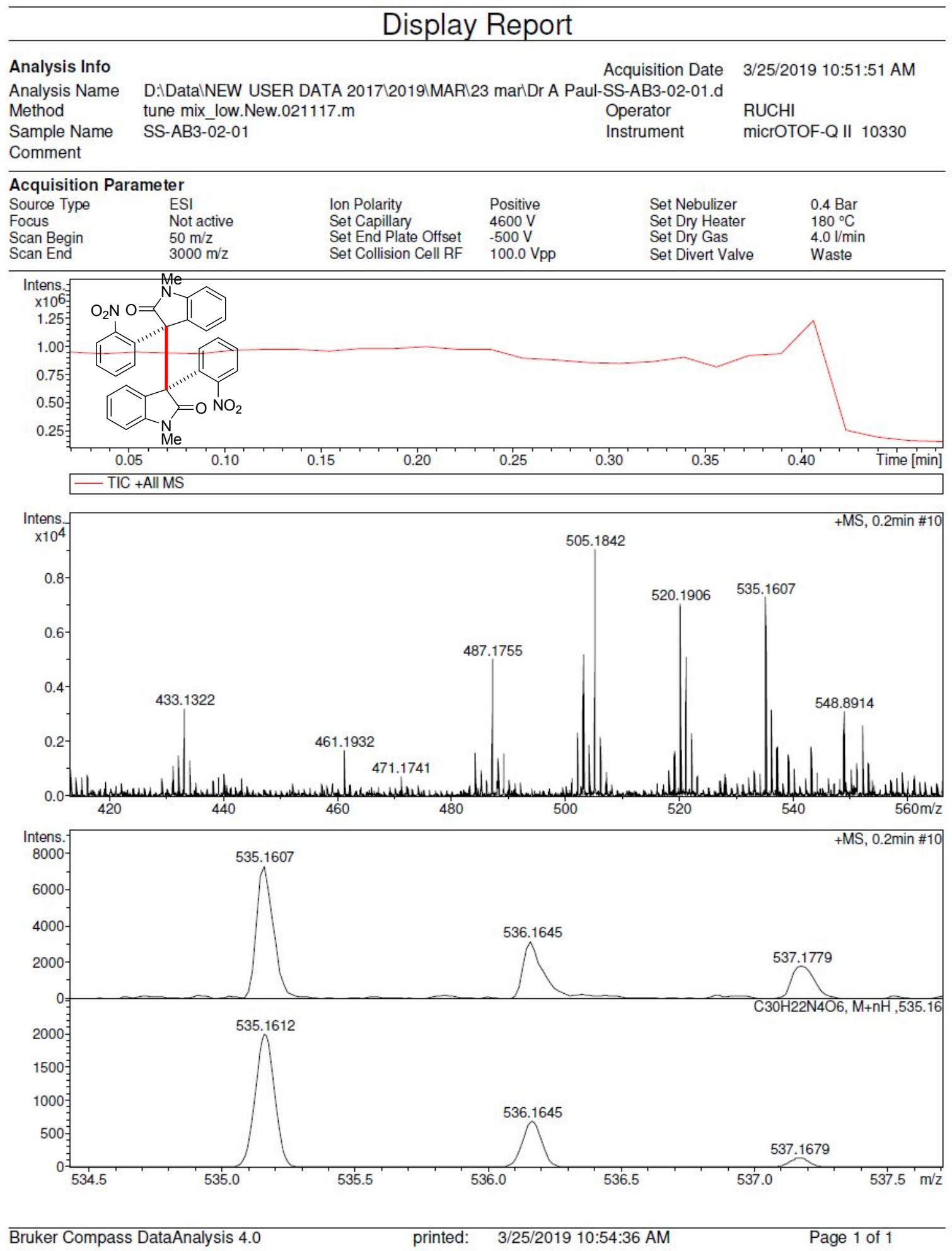

\section{Mass Spectrum of 4n}



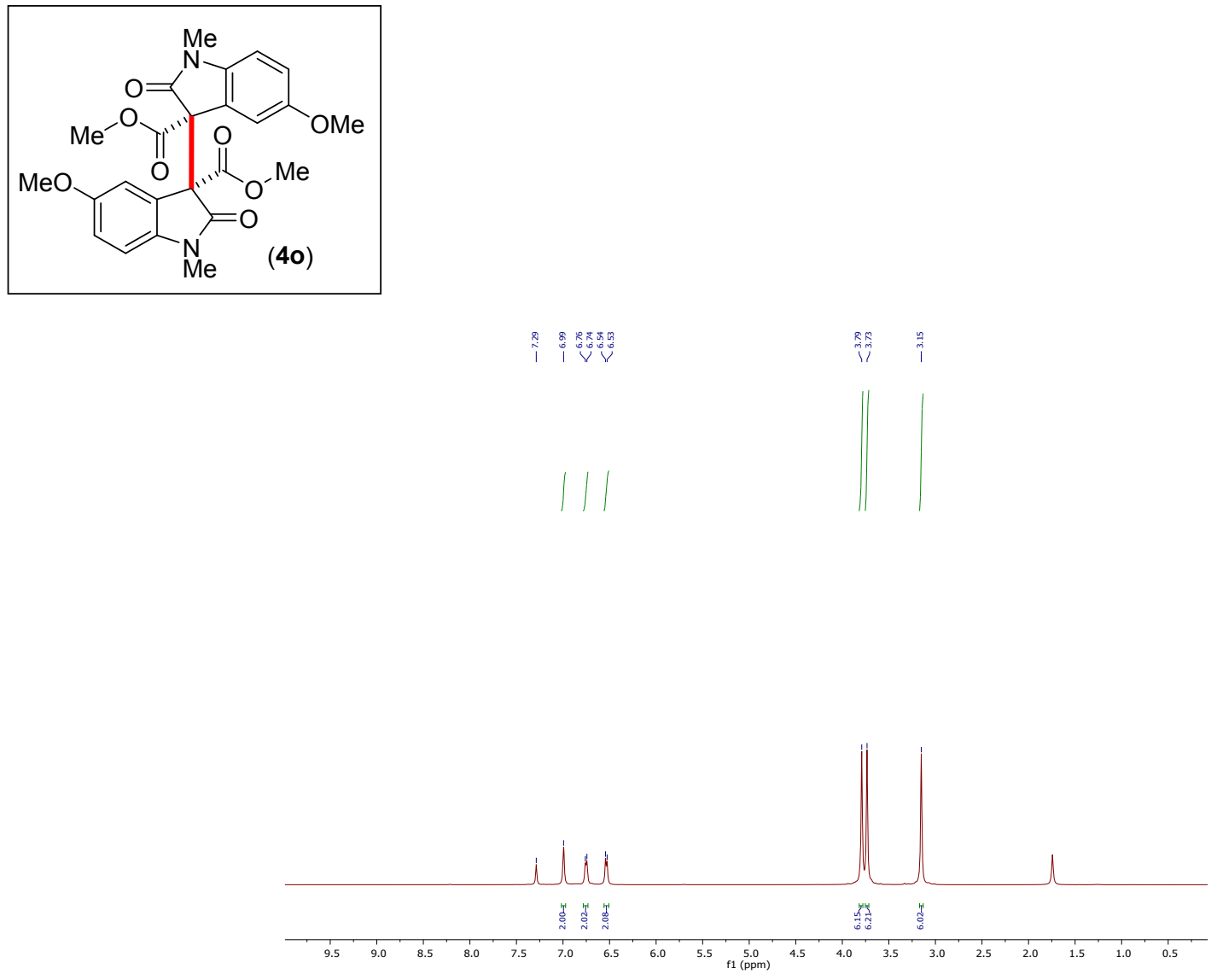

${ }^{1} \mathrm{H}$ NMR $\left(500 \mathrm{MHz}, \mathrm{CDCl}_{3}\right)$ of compound $4 \mathbf{o}$
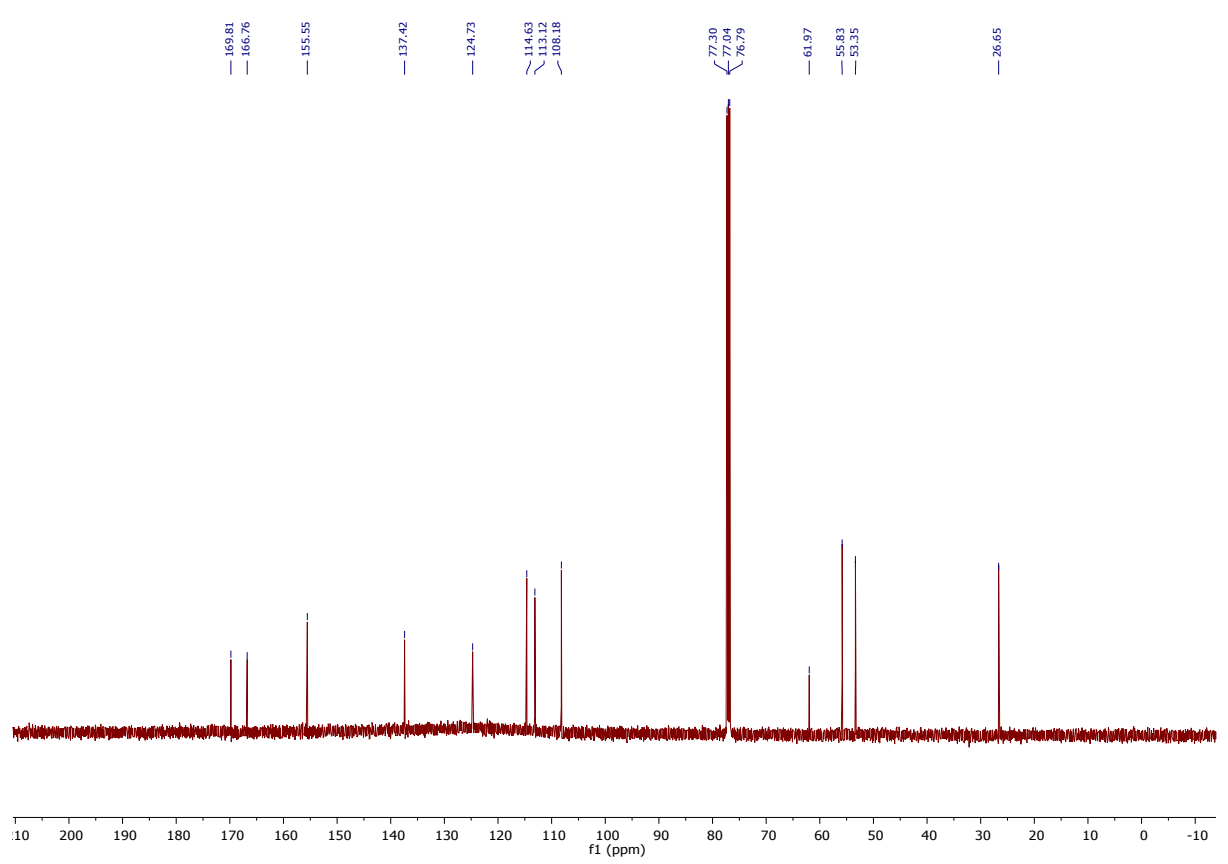

${ }^{13} \mathrm{C}\left\{{ }^{1} \mathrm{H}\right\} \mathrm{NMR}\left(125 \mathrm{MHz}, \mathrm{CDCl}_{3}\right)$ of compound $4 \mathrm{o}$ 
Display Report

Analysis Info

Analysis Name

Method

Sample Name

Comment
Acquisition Date

D:IData|NEW USER DATA 2020 Sept-2020121-sep\Prof.A.Bisai-ABKS02324.d

tune wide.m

ABKS02324
Operator

Instrument
9/21/2020 4:13:04 PM

RUCHI

micrOTOF-Q II 10330
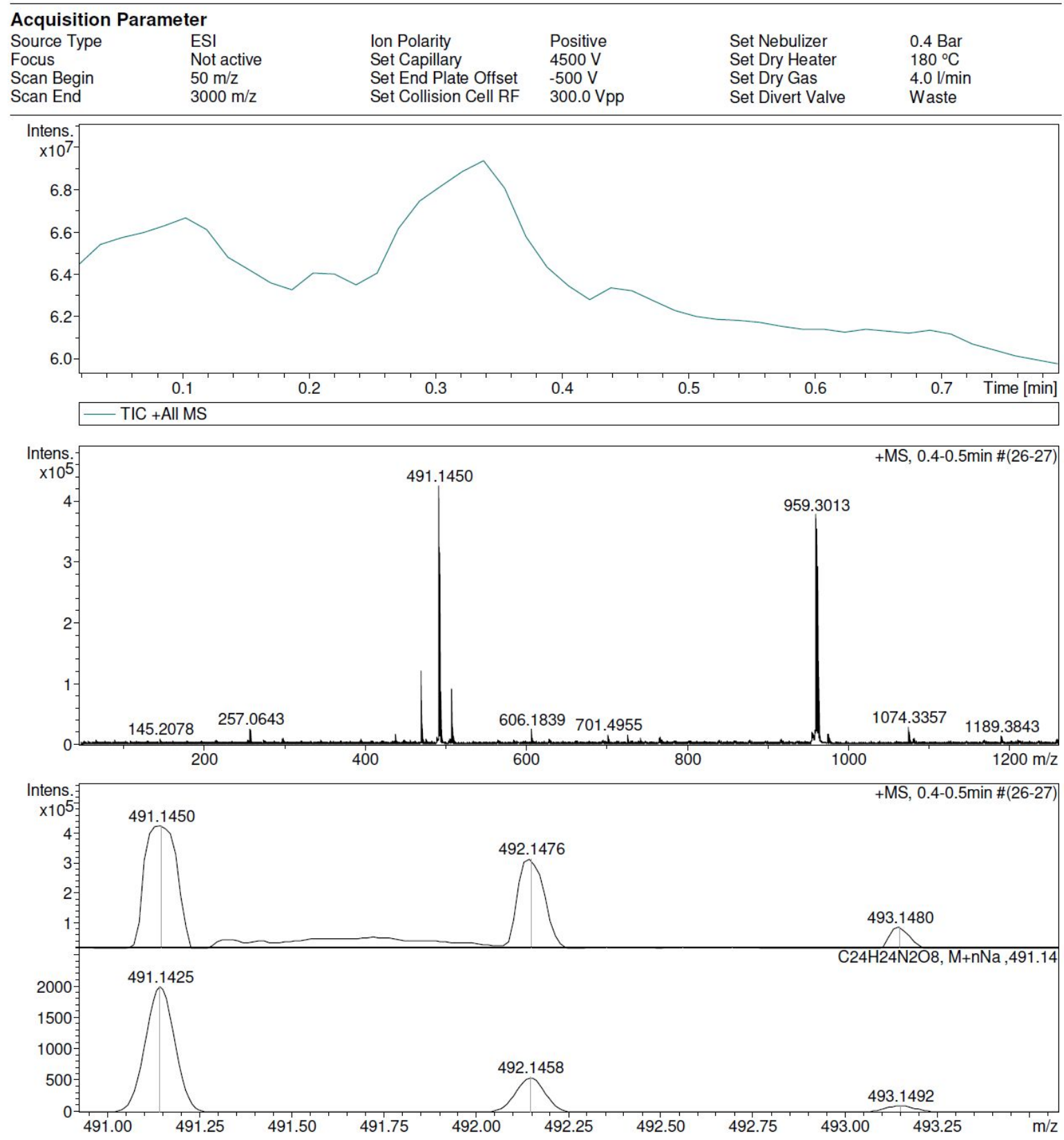

Bruker Compass DataAnalysis 4.0

printed: $\quad$ 9/21/2020 4:14:42 PM

Page 1 of 1

\section{Mass Spectrum of $4 \mathbf{0}$}



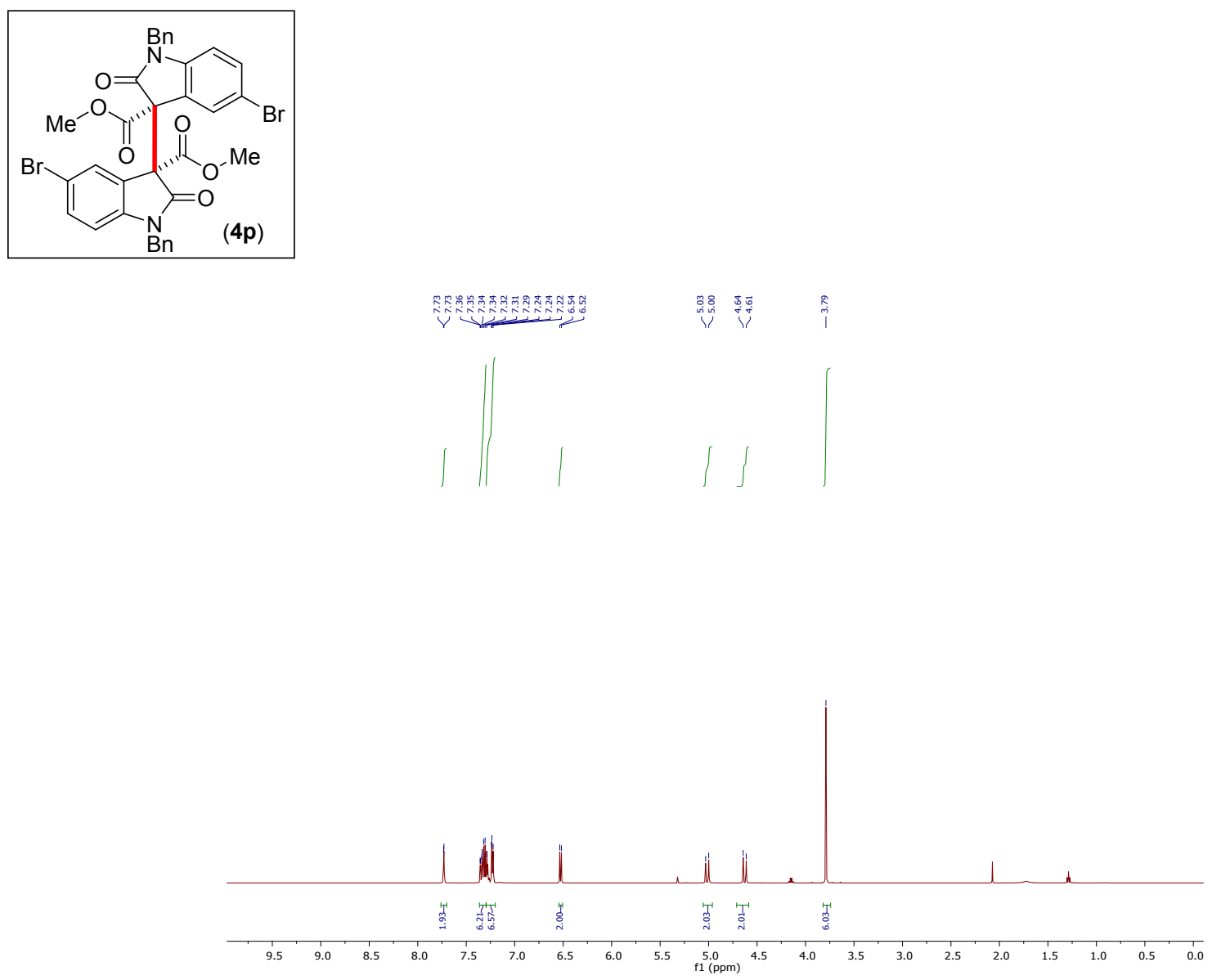

${ }^{1} \mathrm{H}$ NMR $\left(500 \mathrm{MHz}, \mathrm{CDCl}_{3}\right)$ of compound $\mathbf{4 p}$
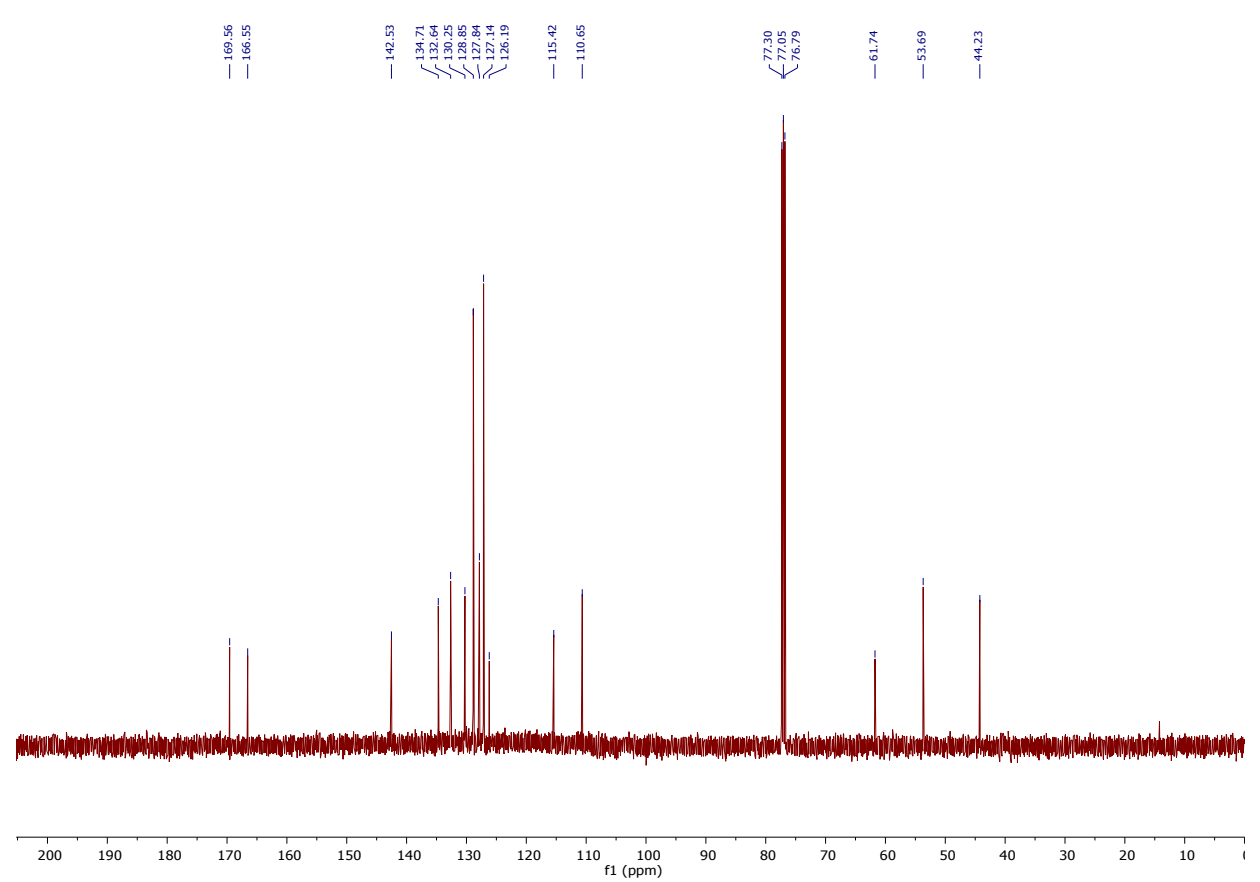

${ }^{13} \mathrm{C}\left\{{ }^{1} \mathrm{H}\right\} \mathrm{NMR}\left(125 \mathrm{MHz}, \mathrm{CDCl}_{3}\right)$ of compound $\mathbf{4 p}$ 


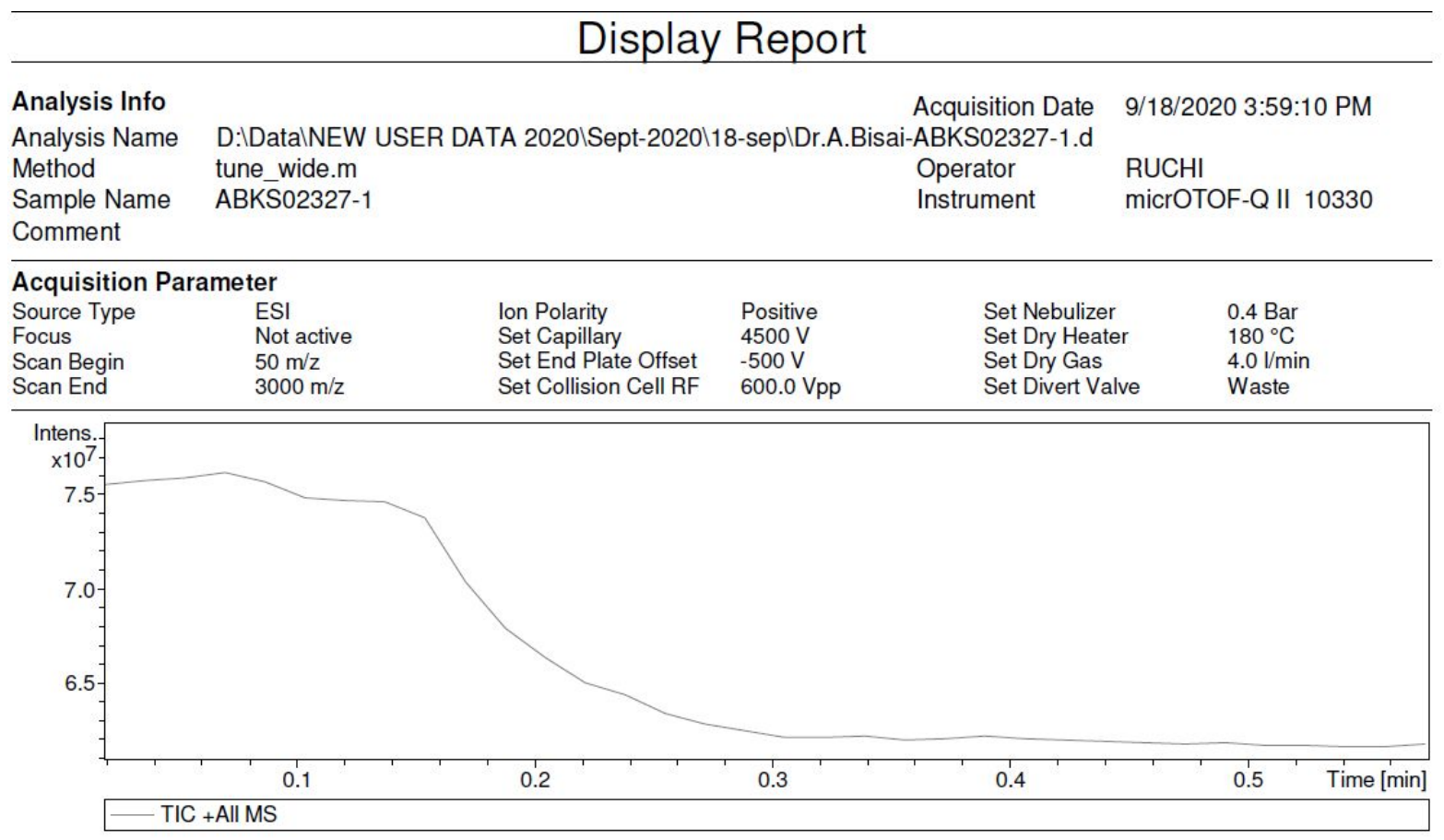
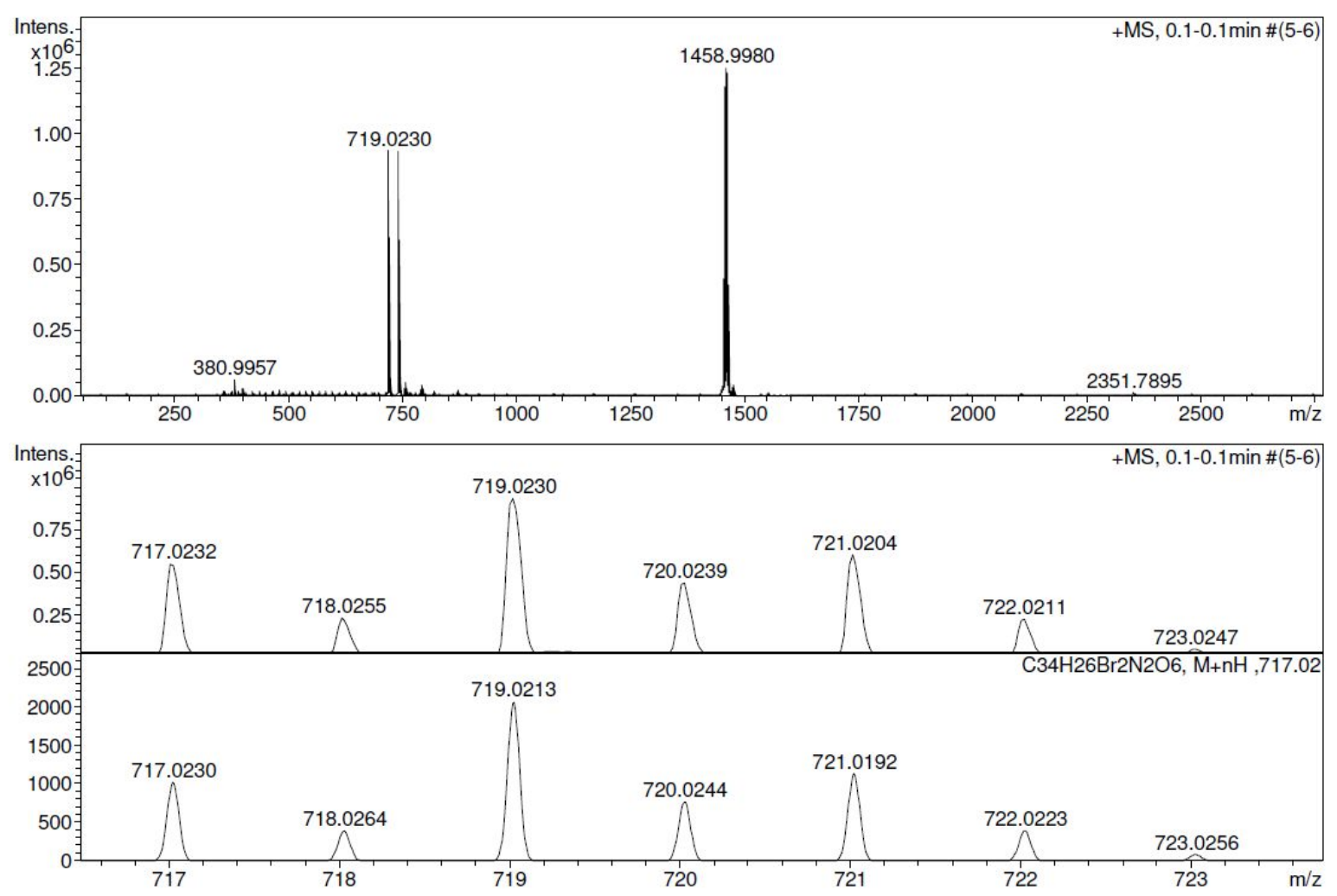

\section{Mass Spectrum of $\mathbf{4 p}$}



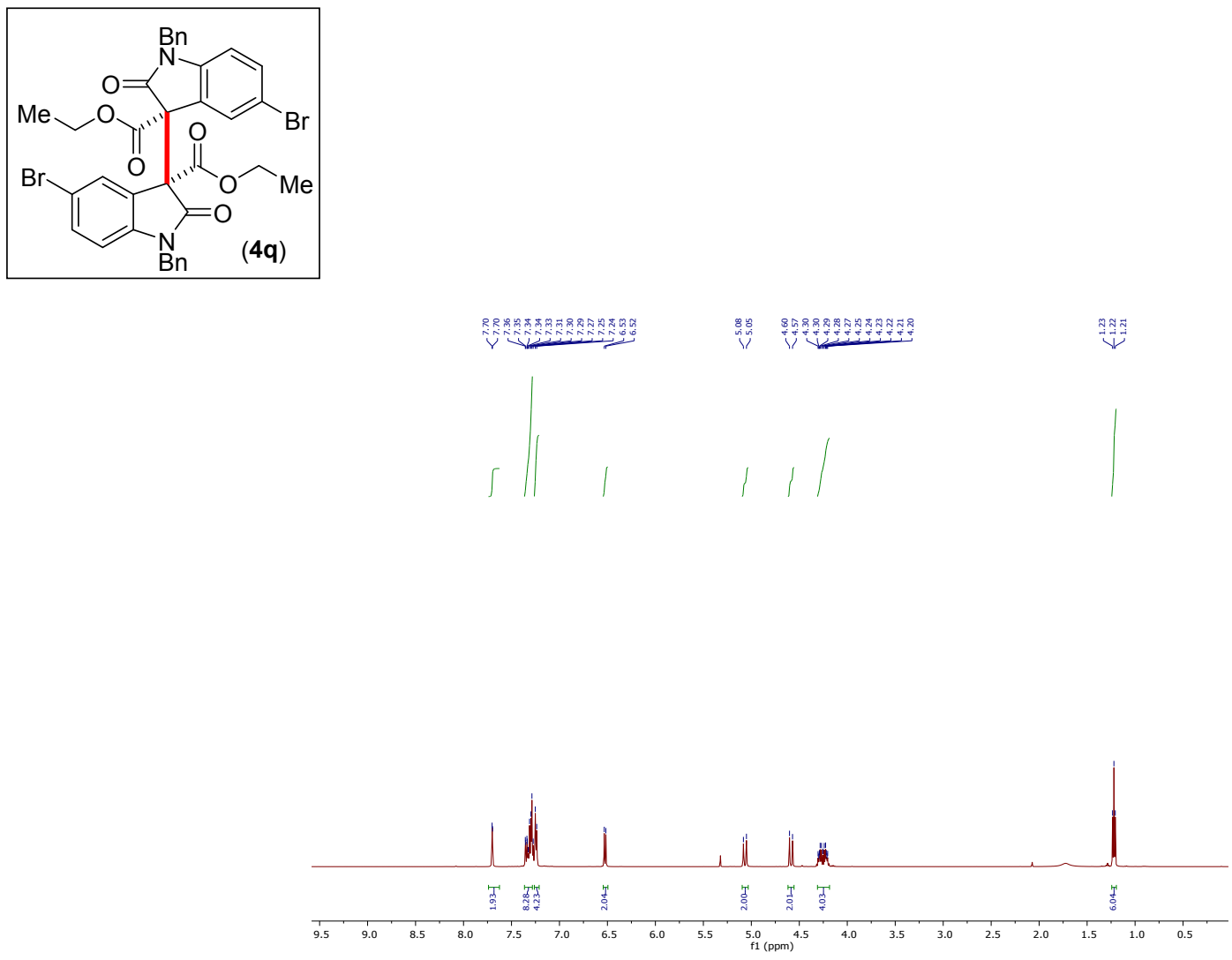

${ }^{1} \mathrm{H}$ NMR $\left(500 \mathrm{MHz}, \mathrm{CDCl}_{3}\right)$ of compound $\mathbf{4 q}$

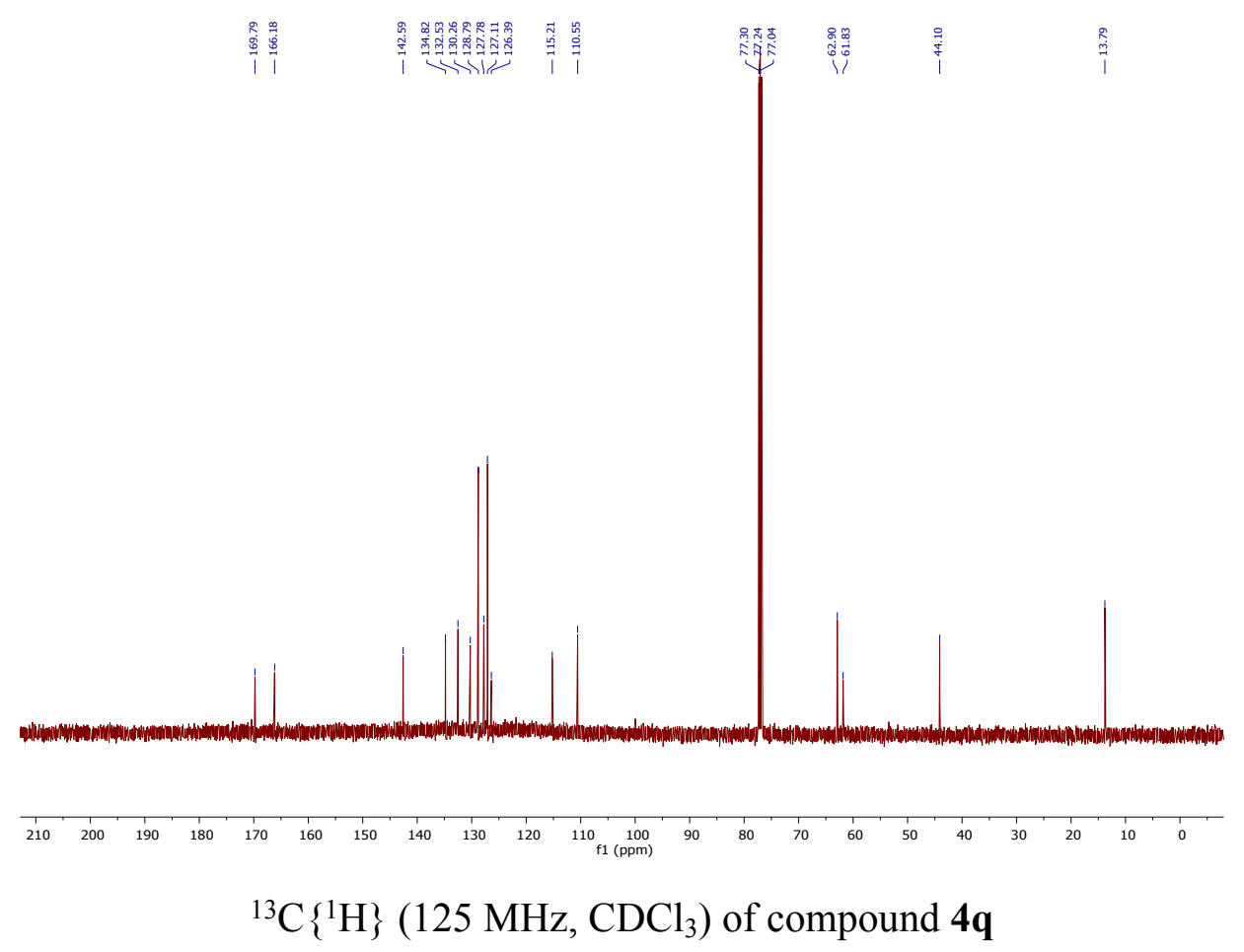




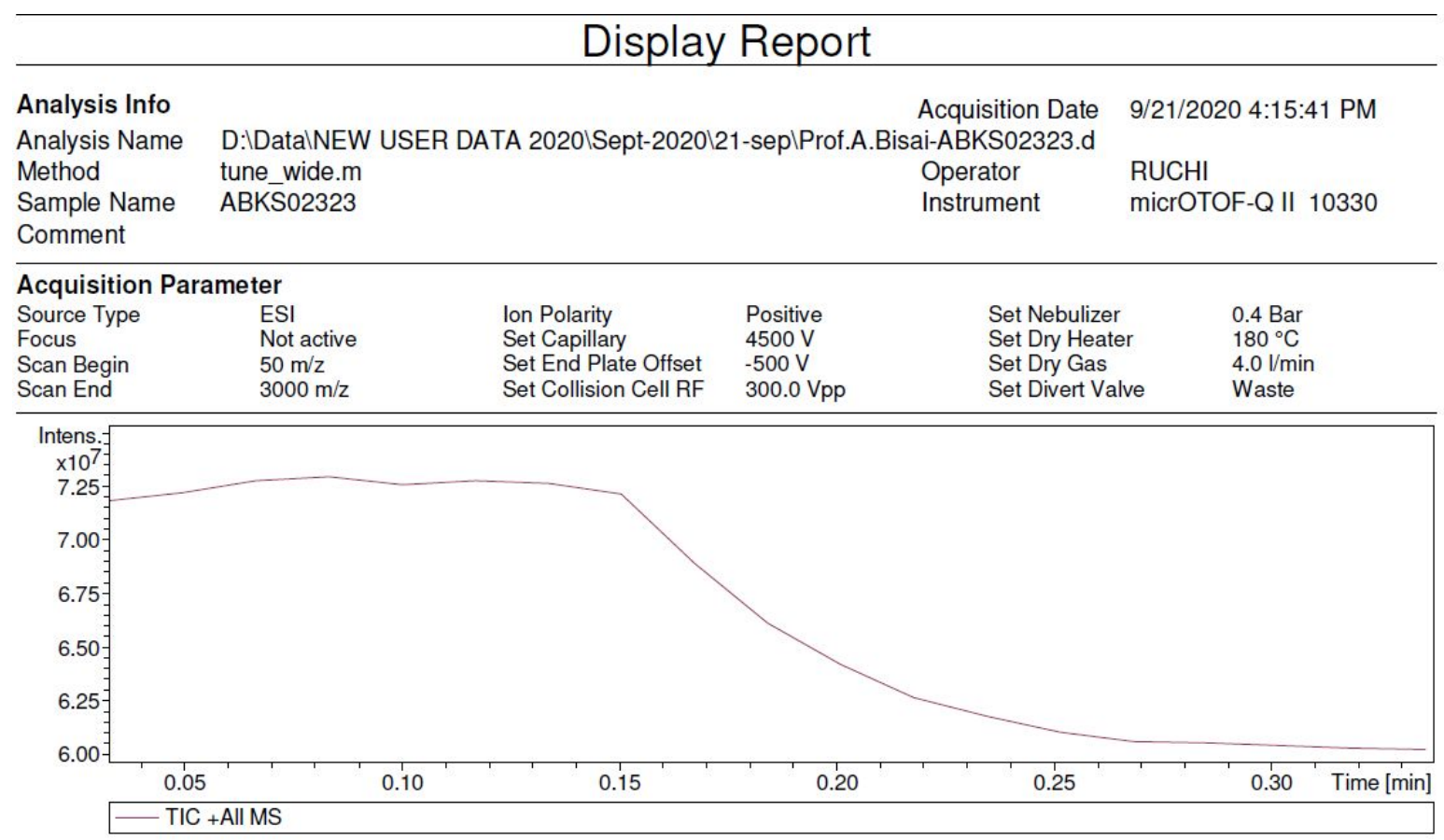
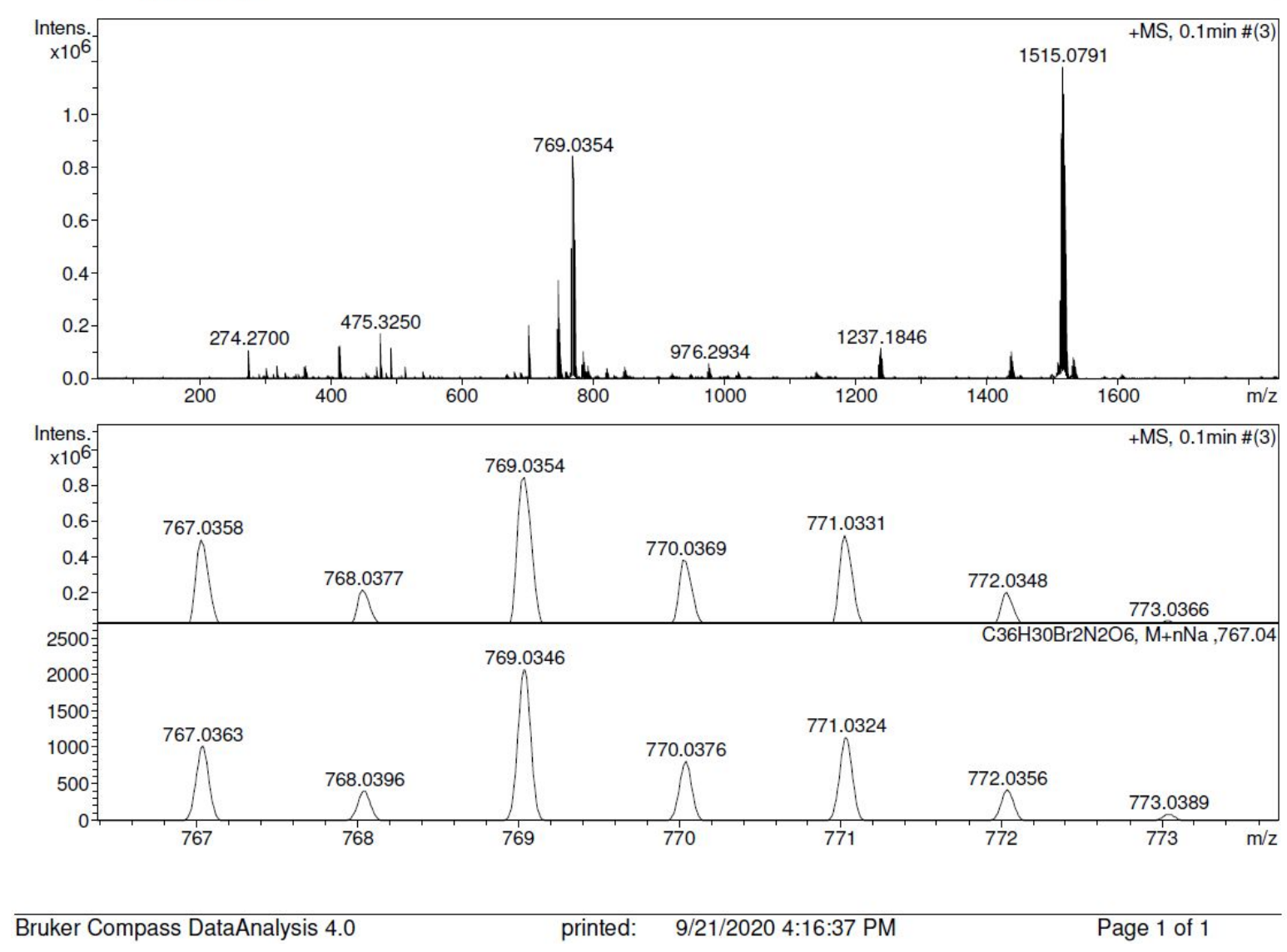

Mass Spectrum of $\mathbf{4 q}$ 

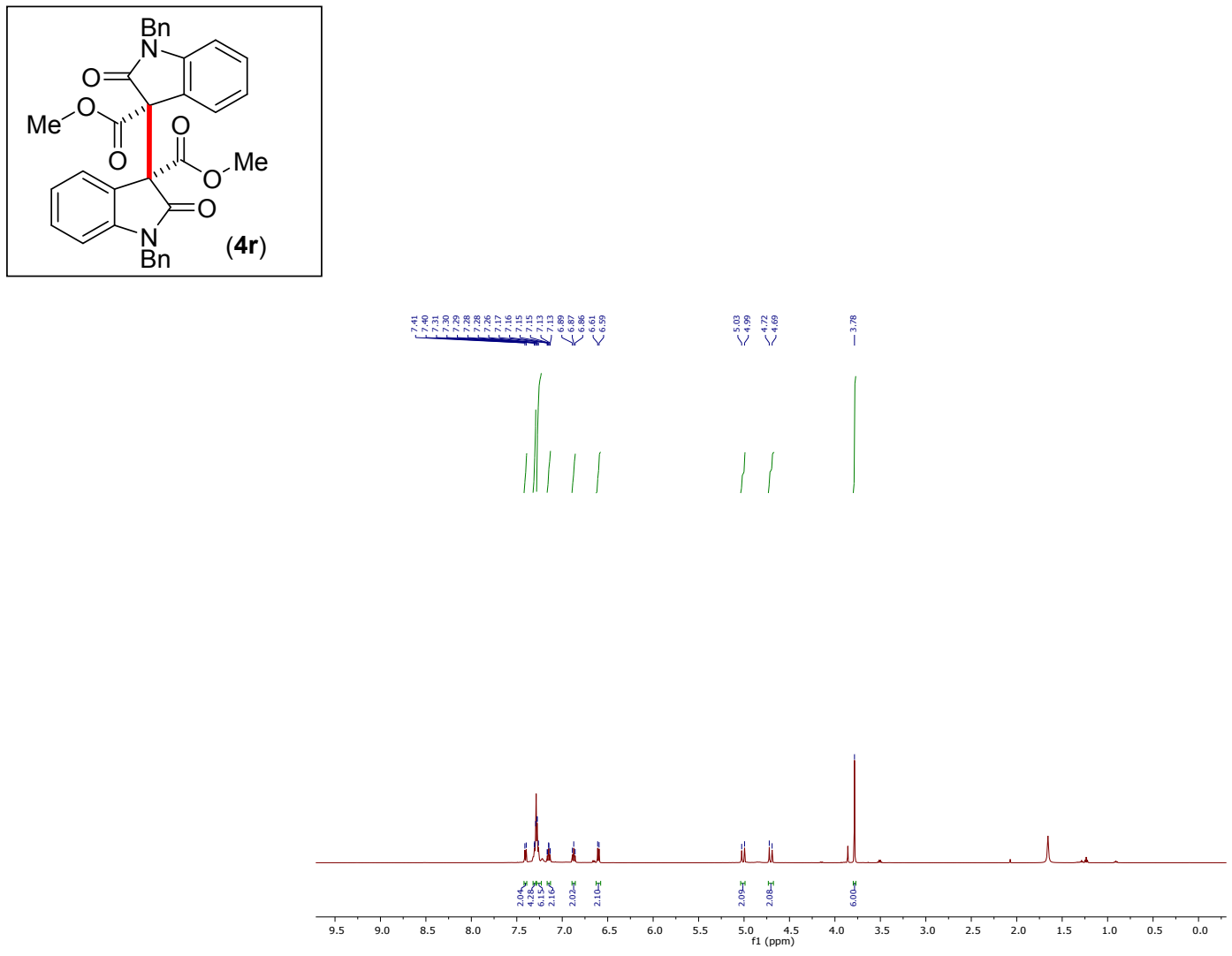

${ }^{1} \mathrm{H} \mathrm{NMR}\left(500 \mathrm{MHz}, \mathrm{CDCl}_{3}\right)$ of compound $4 \mathbf{r}$

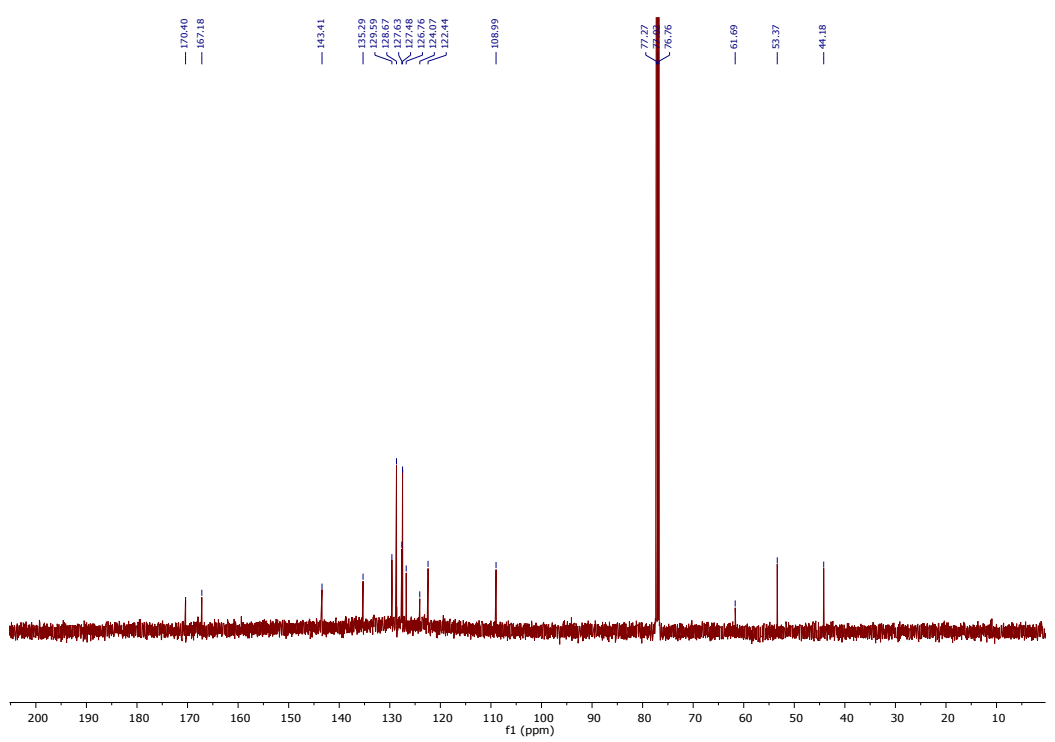

${ }^{13} \mathrm{C}\left\{{ }^{1} \mathrm{H}\right\} \mathrm{NMR}\left(125 \mathrm{MHz}, \mathrm{CDCl}_{3}\right)$ of compound $4 \mathbf{r}$ 
Analysis Info

Analysis Name

Method

Sample Name

Comment
Acquisition Date 9/22/2020 3:50:28 PM

D:IDatalNEW USER DATA 2020।Sept-2020122-sep\Prof.V.K.Singh-ABKS02322.d

tune_low.m

ABKS02322
$\mathrm{RUCHI}$

Instrument micrOTOF-Q || 10330
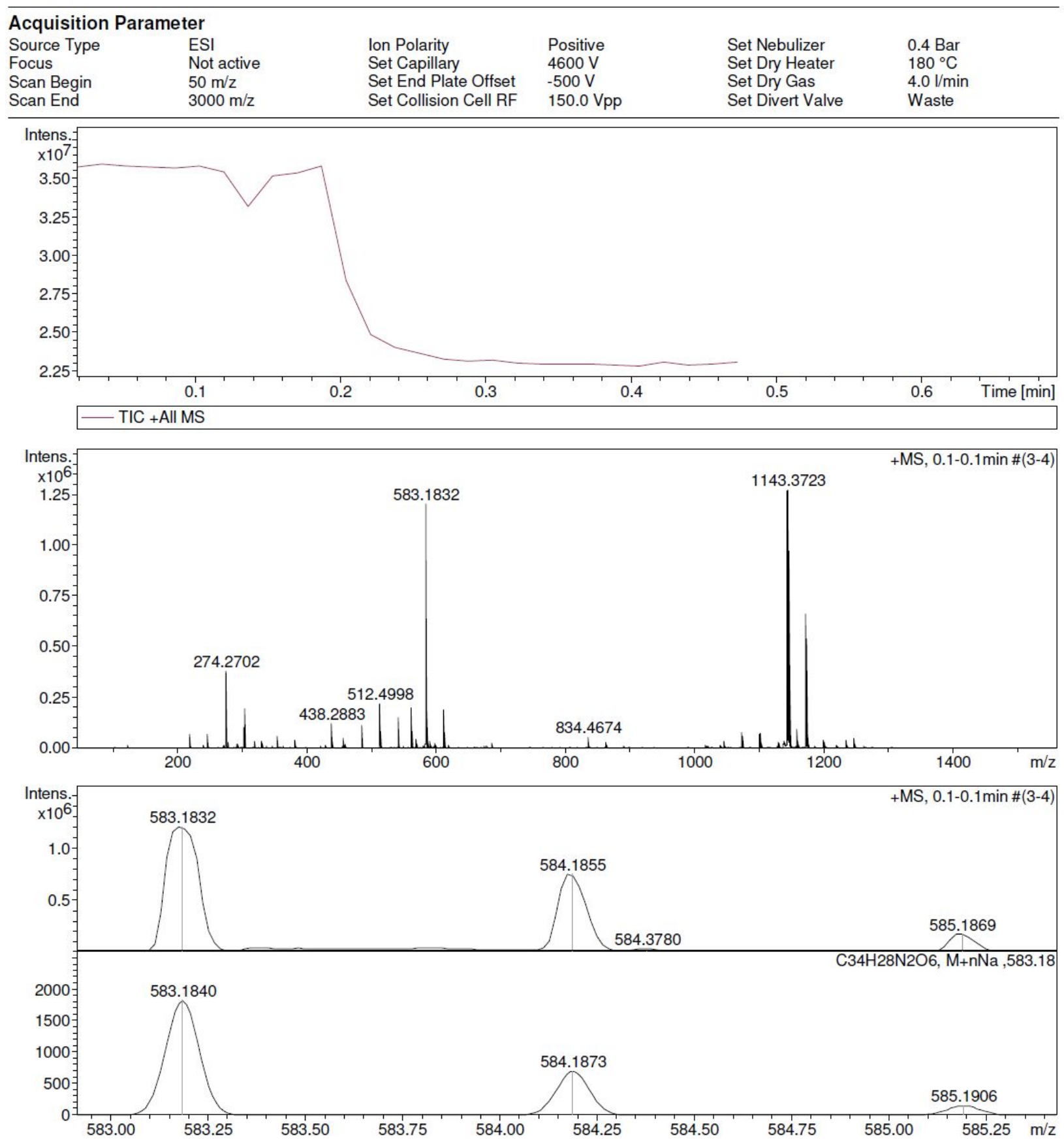

\section{Mass Spectrum of $\mathbf{4 r}$}



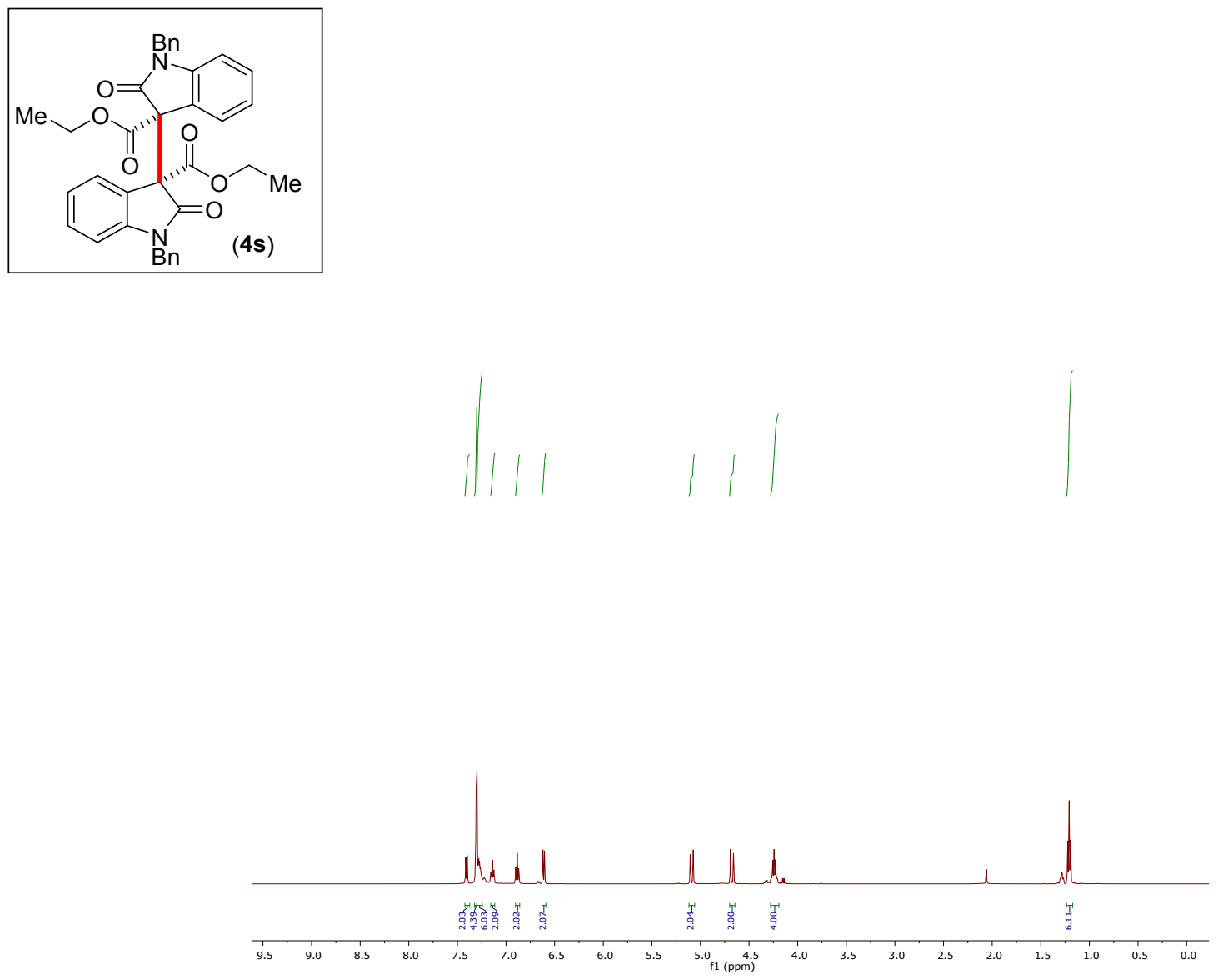

${ }^{1} \mathrm{H} \mathrm{NMR}\left(500 \mathrm{MHz}, \mathrm{CDCl}_{3}\right)$ of compound $4 \mathbf{s}$
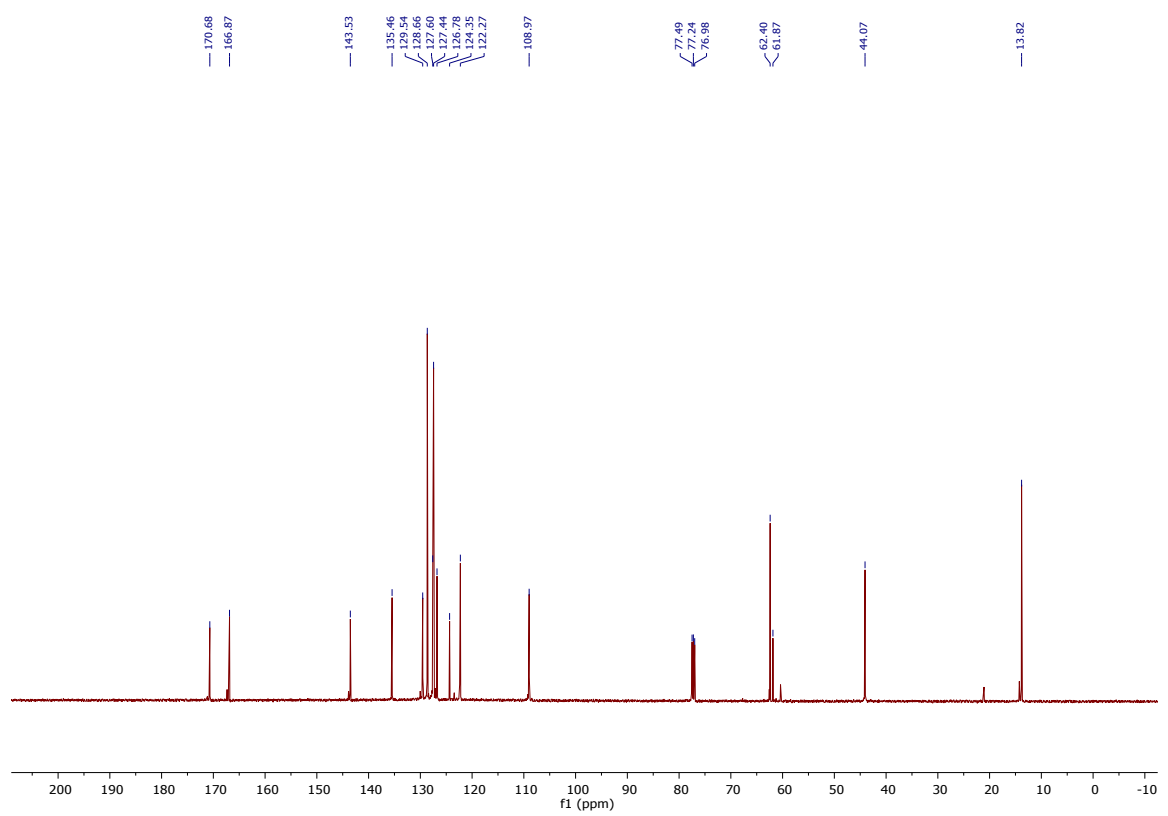

${ }^{13} \mathrm{C}\left\{{ }^{1} \mathrm{H}\right\} \mathrm{NMR}\left(125 \mathrm{MHz}, \mathrm{CDCl}_{3}\right)$ of compound $\mathbf{4 s}$ 
Analysis Info

Analysis Name

Method

Sample Name

Comment
Acquisition Date 9/22/2020 3:47:50 PM

D:IDatalNEW USER DATA 2020।Sept-2020122-sep\Prof.V.K.Singh-ABKS02321.d

tune_low.m

ABKS02321
$\mathrm{RUCHI}$

Instrument micrOTOF-Q || 10330
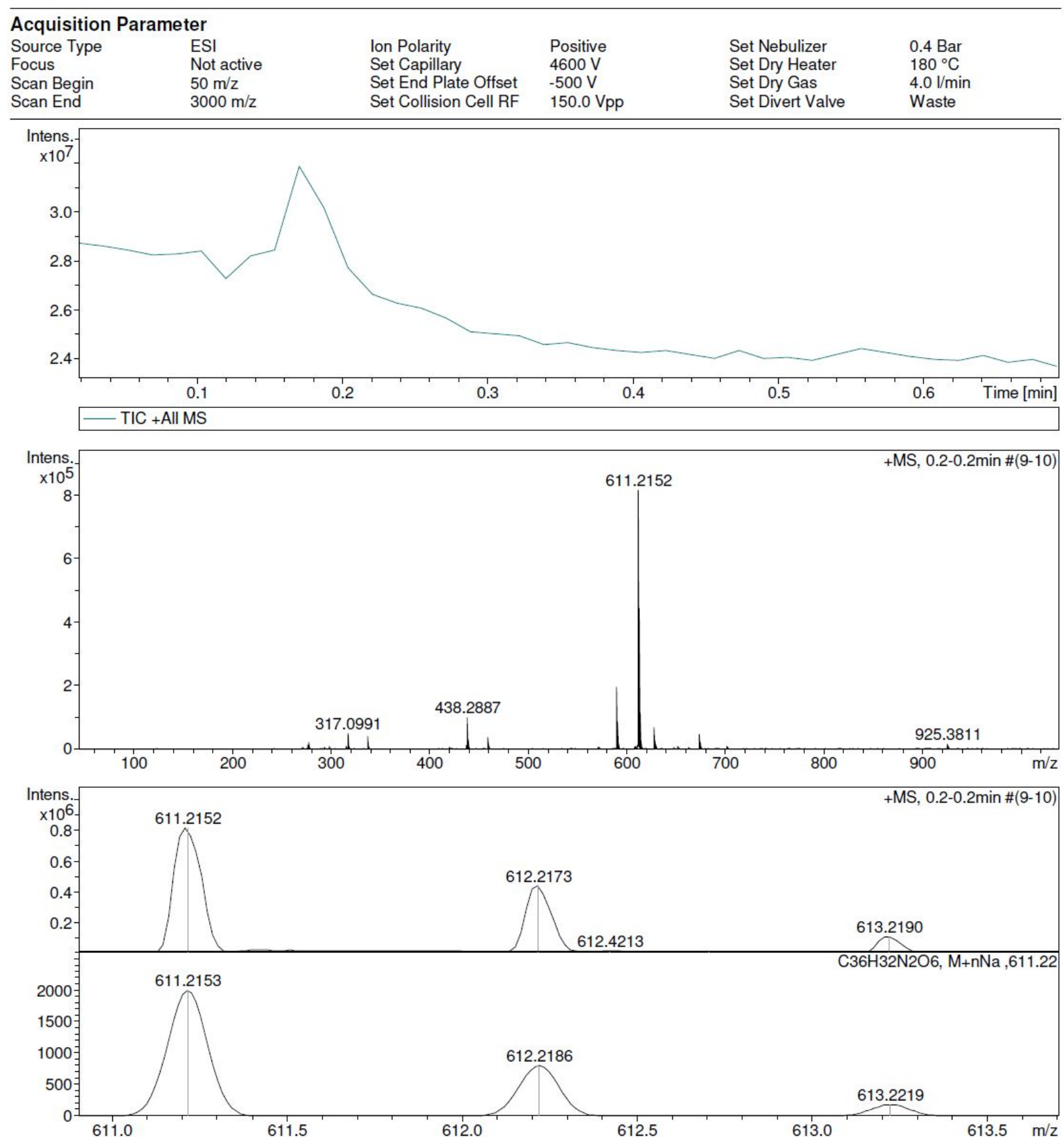

\section{Mass Spectrum of $\mathbf{4 s}$}



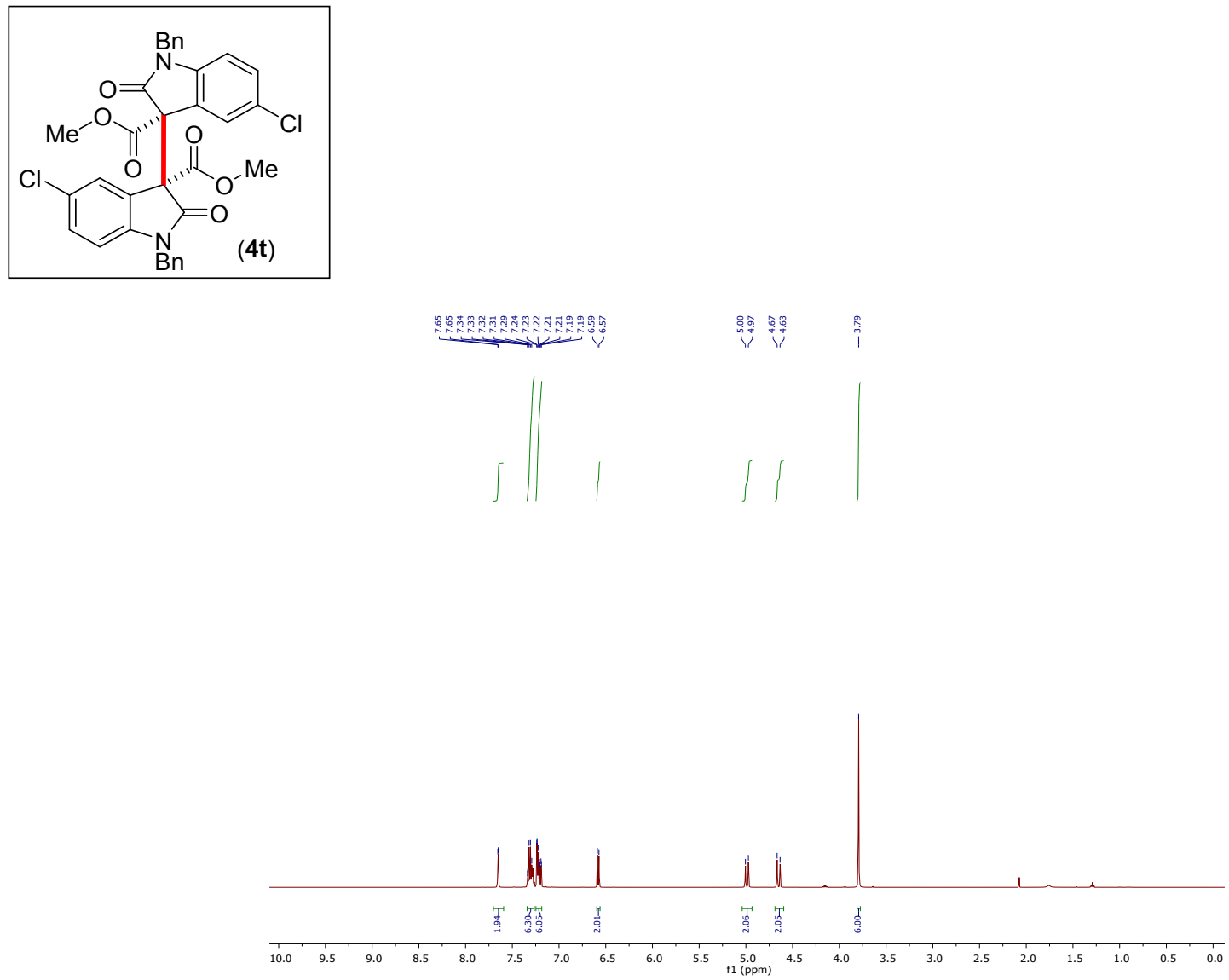

${ }^{1} \mathrm{H}$ NMR $\left(500 \mathrm{MHz}, \mathrm{CDCl}_{3}\right)$ of compound $\mathbf{4 t}$

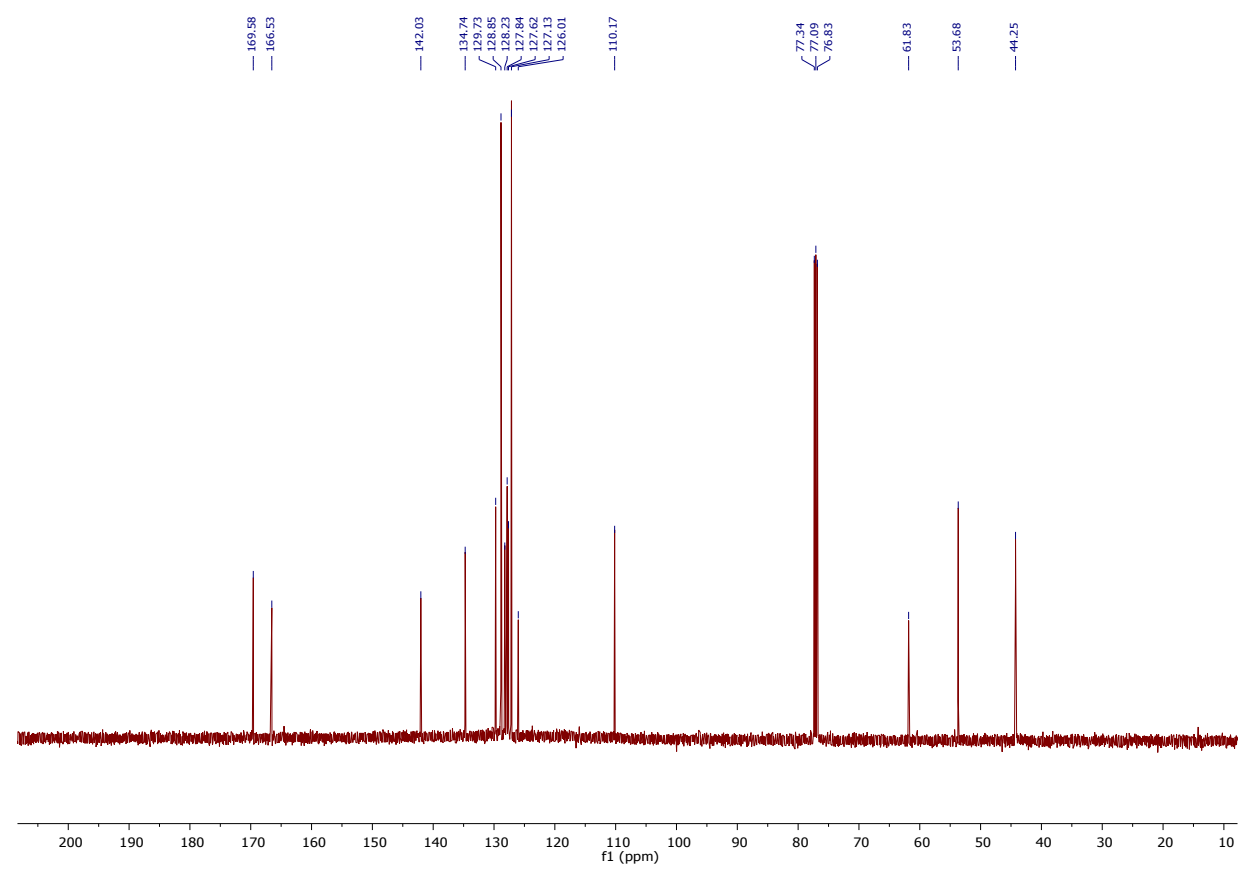

${ }^{13} \mathrm{C}\left\{{ }^{1} \mathrm{H}\right\}$ NMR $\left(125 \mathrm{MHz}, \mathrm{CDCl}_{3}\right)$ of compound $\mathbf{4 t}$ 
Analysis Info

Analysis Name

Method

Sample Name

Comment
Acquisition Date 9/23/2020 4:32:40 PM

D:IDatalNEW USER DATA 2020।Sept-2020\23-seplDr.A.Paul-ABKS02319-1.d

tune_wide.m

ABKS02319-1
Operator

Instrument
RUCHI

micrOTOF-Q II 10330
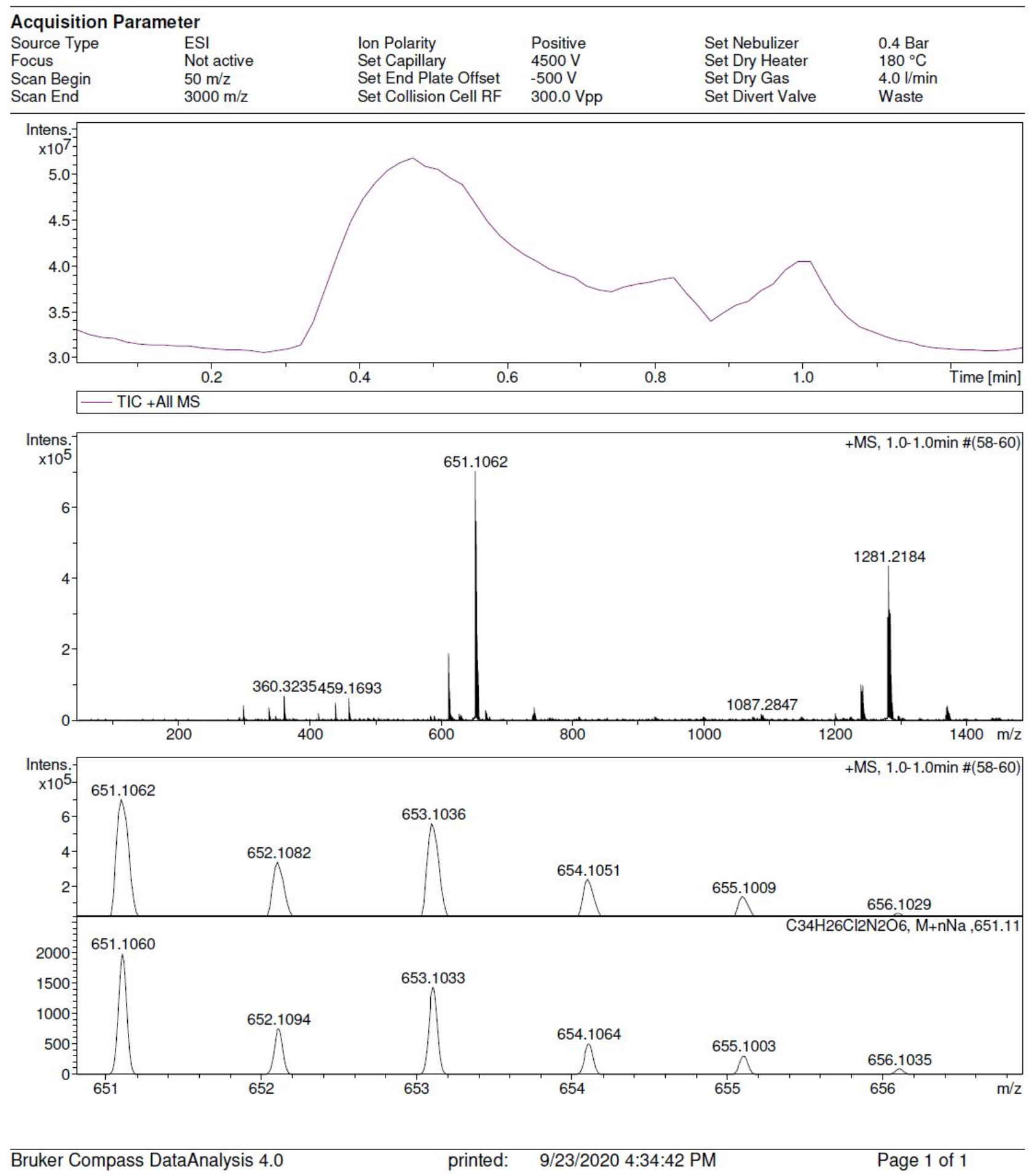

\section{Mass Spectrum of $\mathbf{4 t}$}



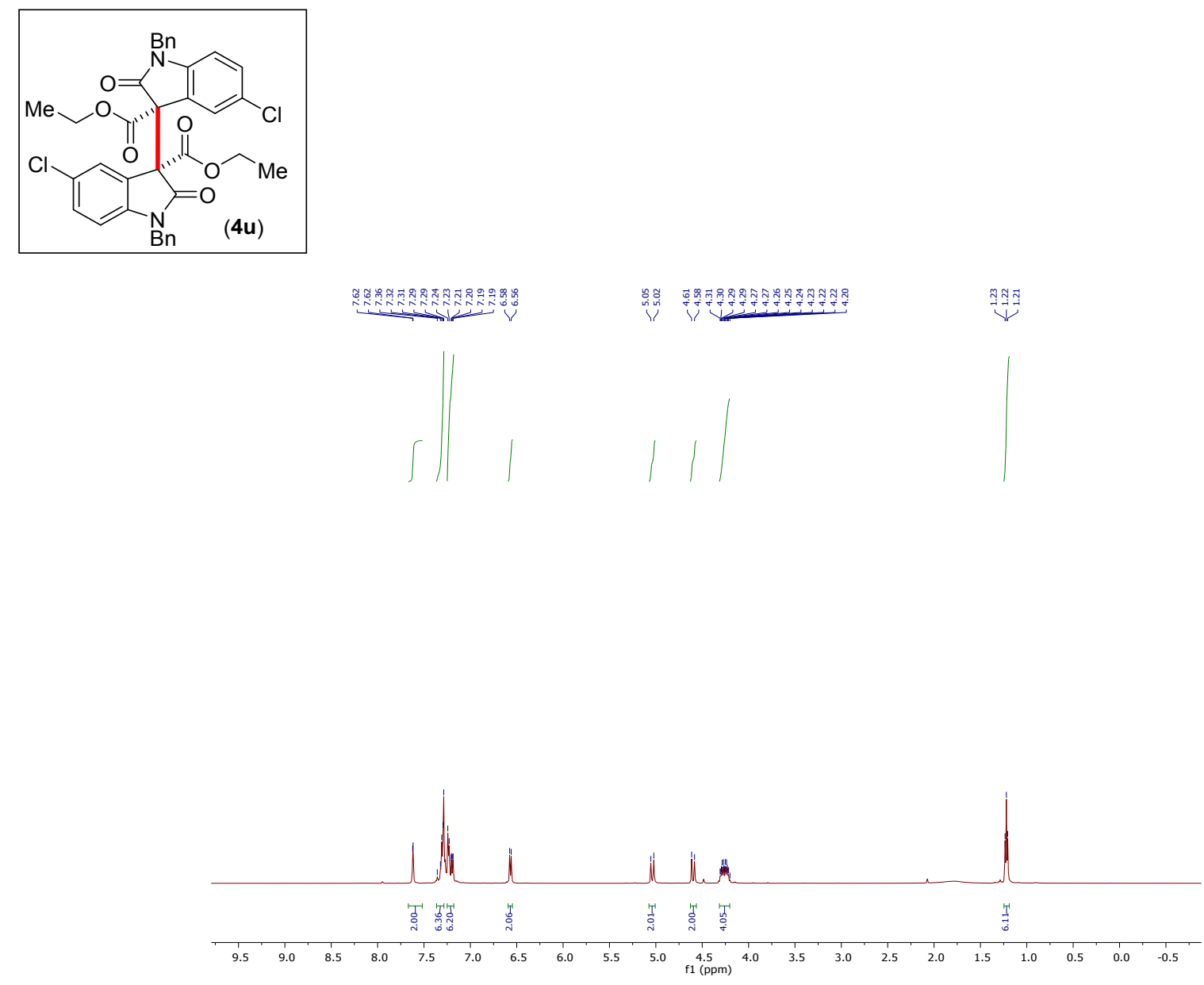

${ }^{1} \mathrm{H}$ NMR $\left(500 \mathrm{MHz}, \mathrm{CDCl}_{3}\right)$ of compound $\mathbf{4 u}$

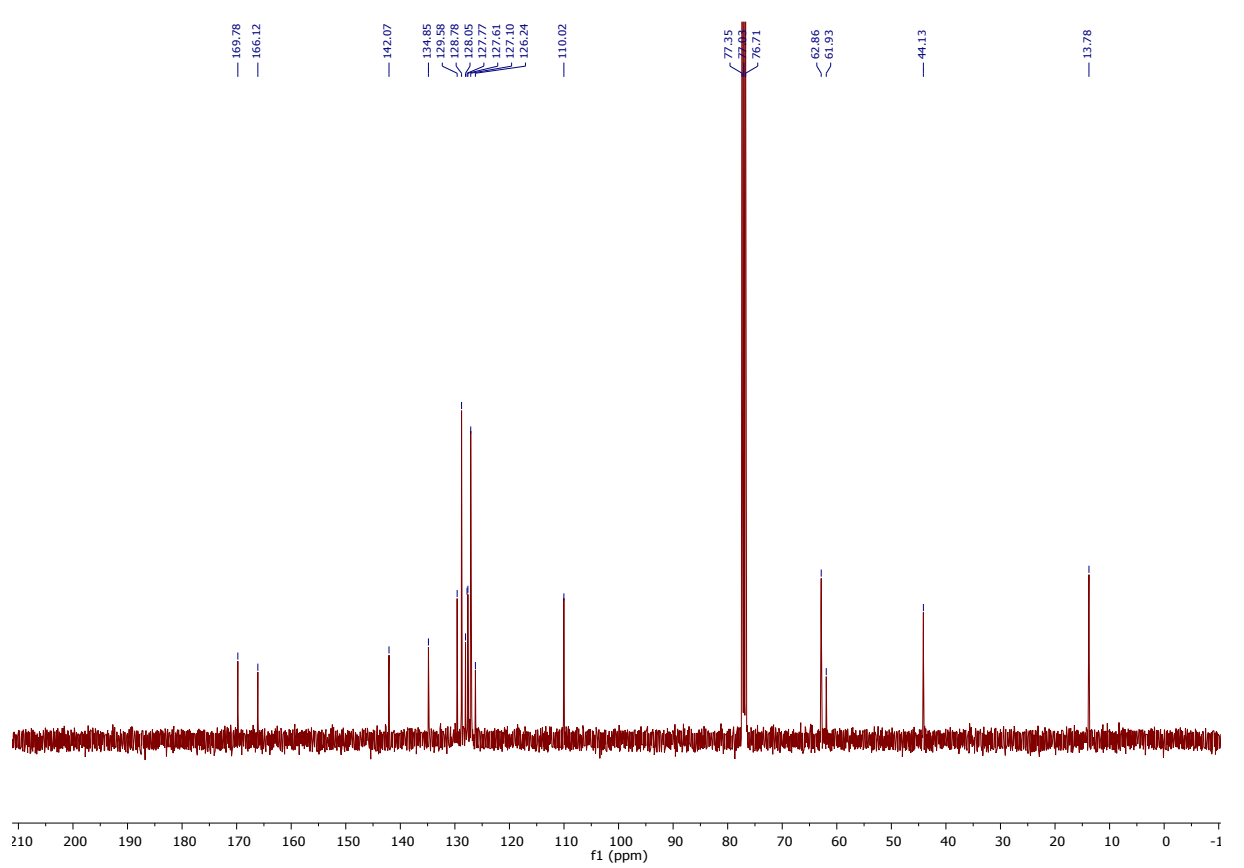

${ }^{13} \mathrm{C}\left\{{ }^{1} \mathrm{H}\right\}$ NMR $\left(100 \mathrm{MHz}, \mathrm{CDCl}_{3}\right)$ of compound $\mathbf{4} \mathbf{u}$ 
Analysis Info

Analysis Name

Method

Sample Name

Comment
Acquisition Date 9/23/2020 4:36:29 PM

D:IDatalNEW USER DATA 2020।Sept-2020123-seplDr.A.Paul-ABKS02320.d

tune_wide.m

ABKS02320

Operator

Instrument

$\mathrm{RUCHI}$

micrOTOF-Q II 10330
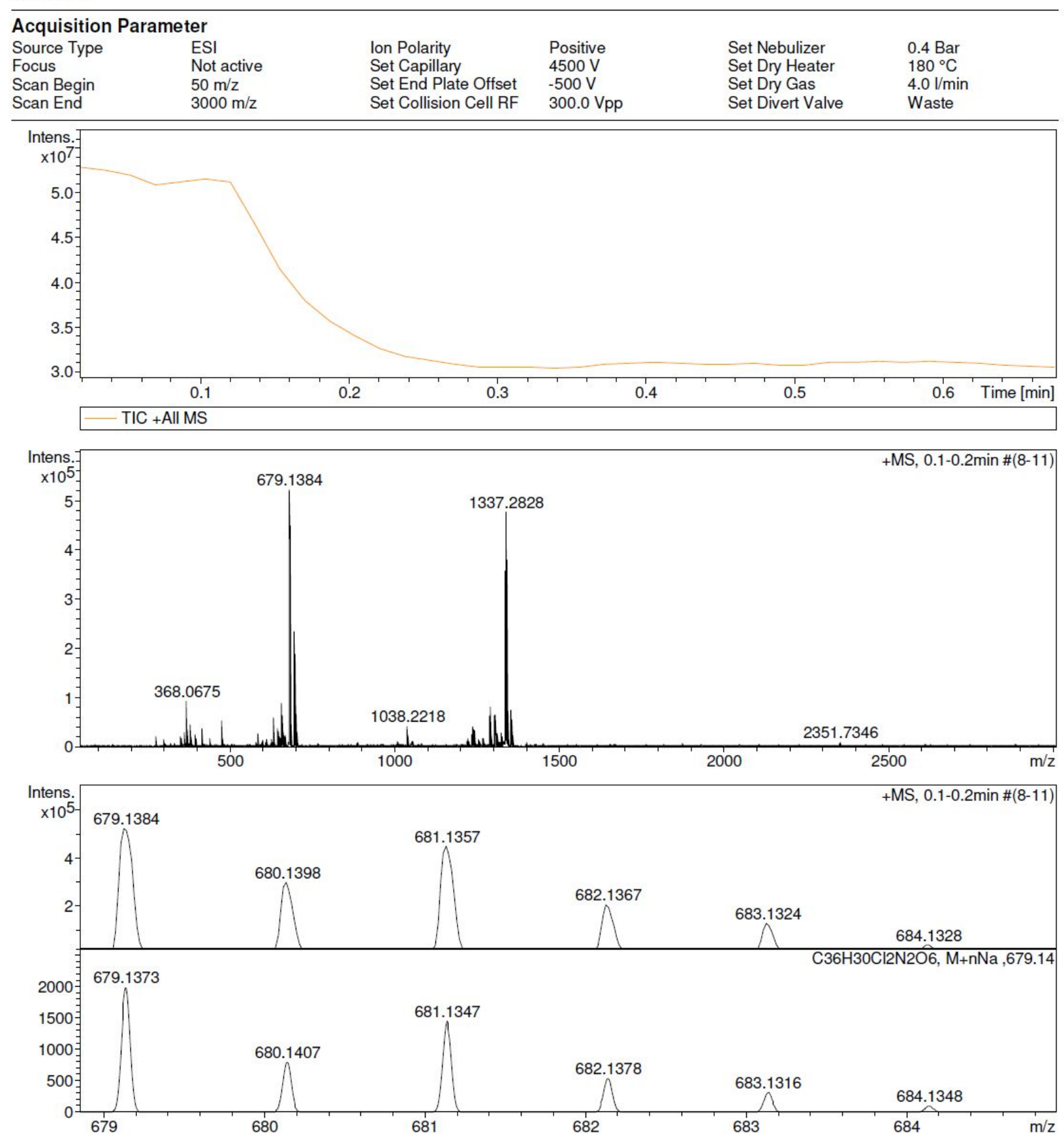

Bruker Compass DataAnalysis 4.0

printed: $\quad$ 9/23/2020 4:38:09 PM

Page 1 of 1

\section{Mass Spectrum of $\mathbf{4 u}$}



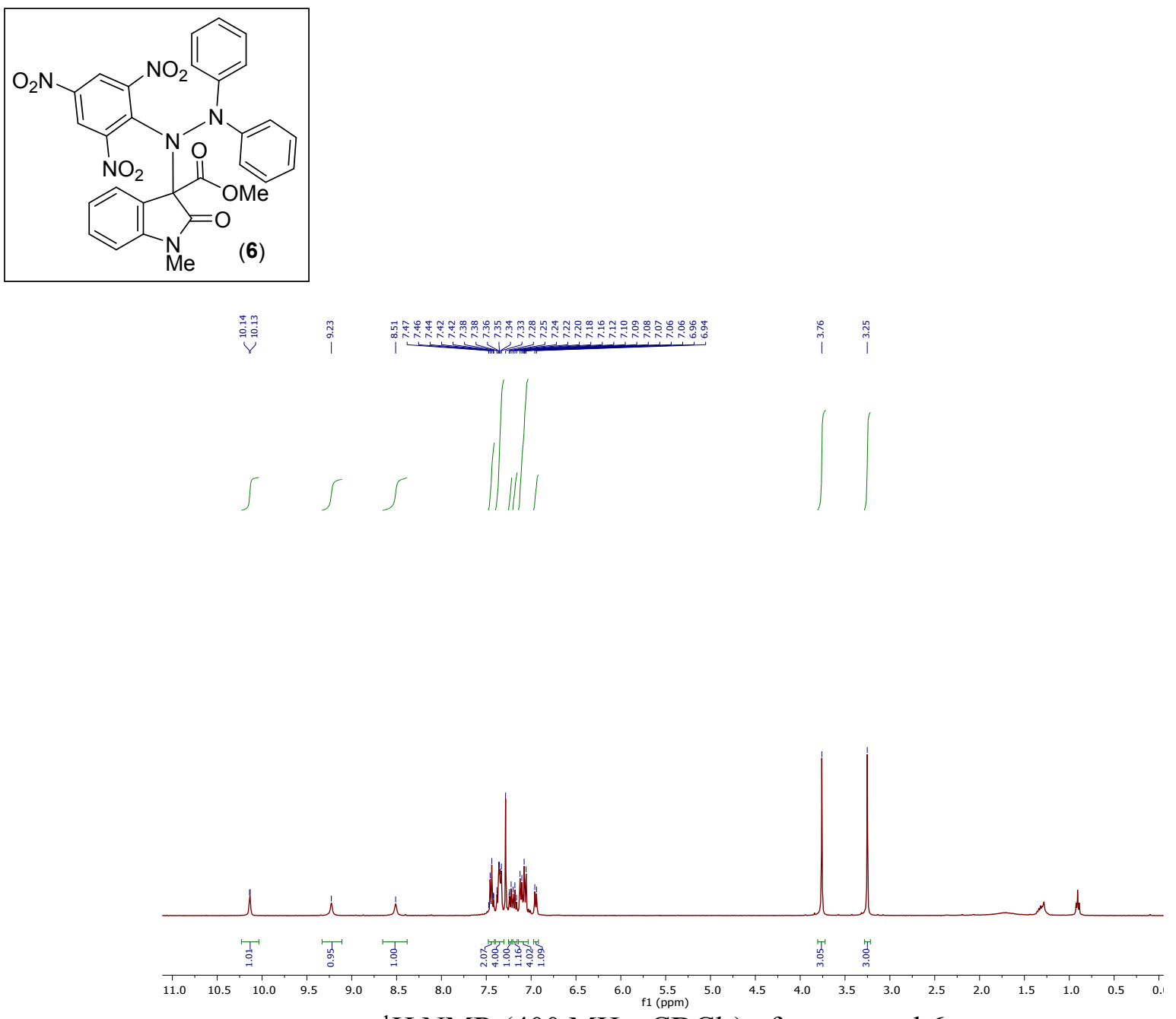

${ }^{1} \mathrm{H} \mathrm{NMR}\left(400 \mathrm{MHz}, \mathrm{CDCl}_{3}\right)$ of compound 6

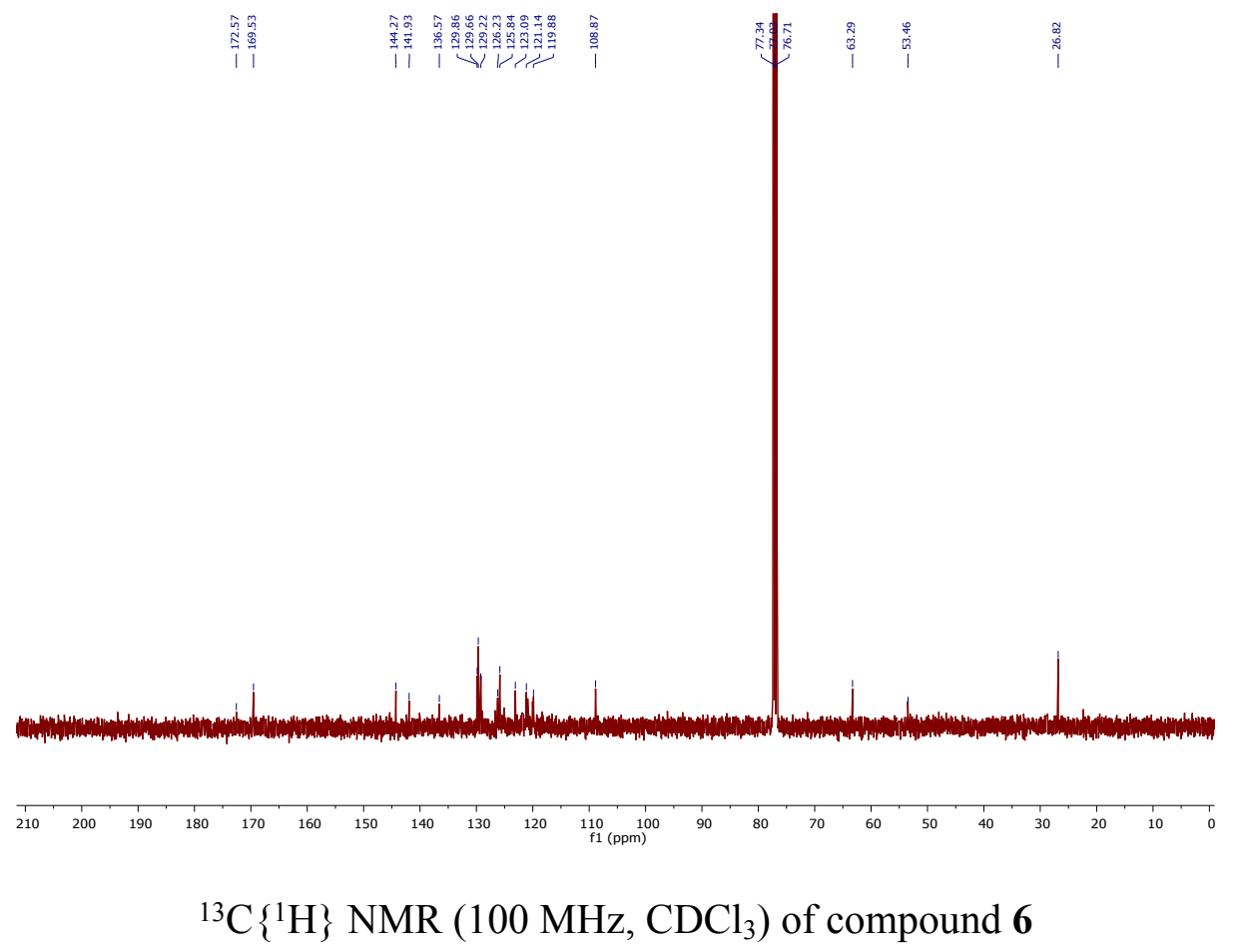




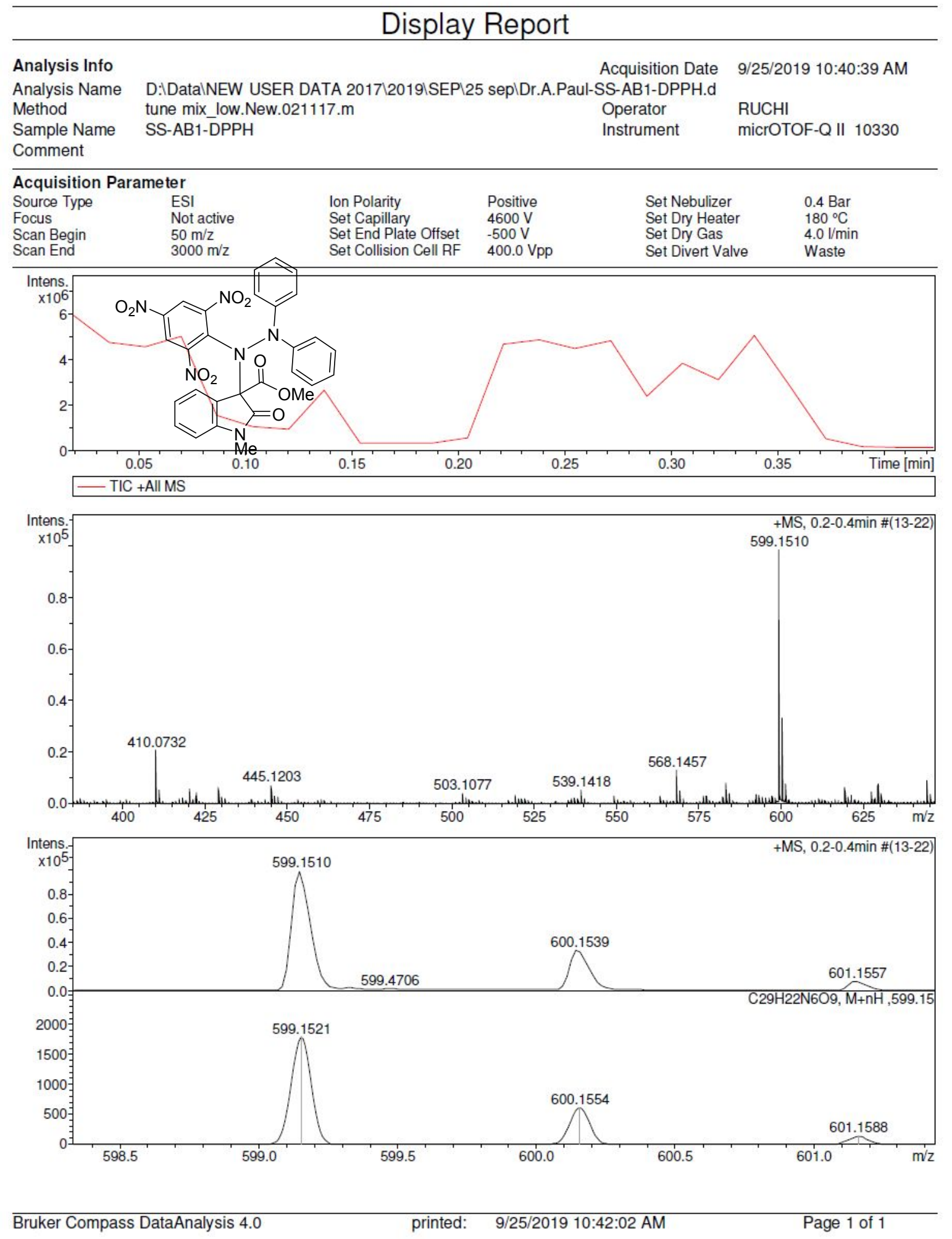

\section{Mass Spectrum of $\mathbf{6}$}


Determination of diastereomeric ratio of (4b-u) from ${ }^{1} \mathrm{H}-\mathrm{NMR}$ of crude reaction mixture:
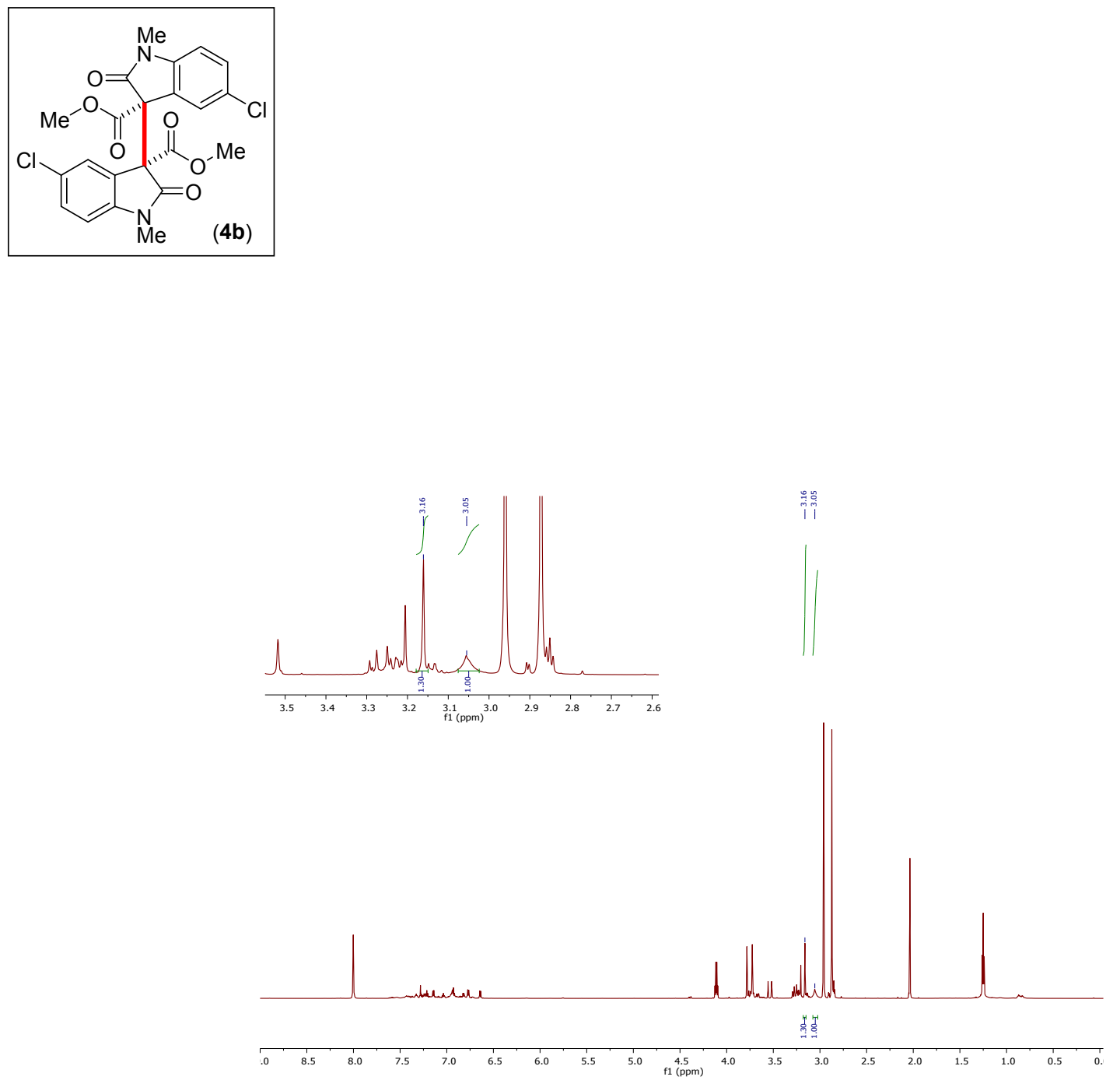

${ }^{1} \mathrm{H}$ NMR $\left(400 \mathrm{MHz}, \mathrm{CDCl}_{3}\right)$ of crude reaction mixture of $(\mathbf{4 b})$ 

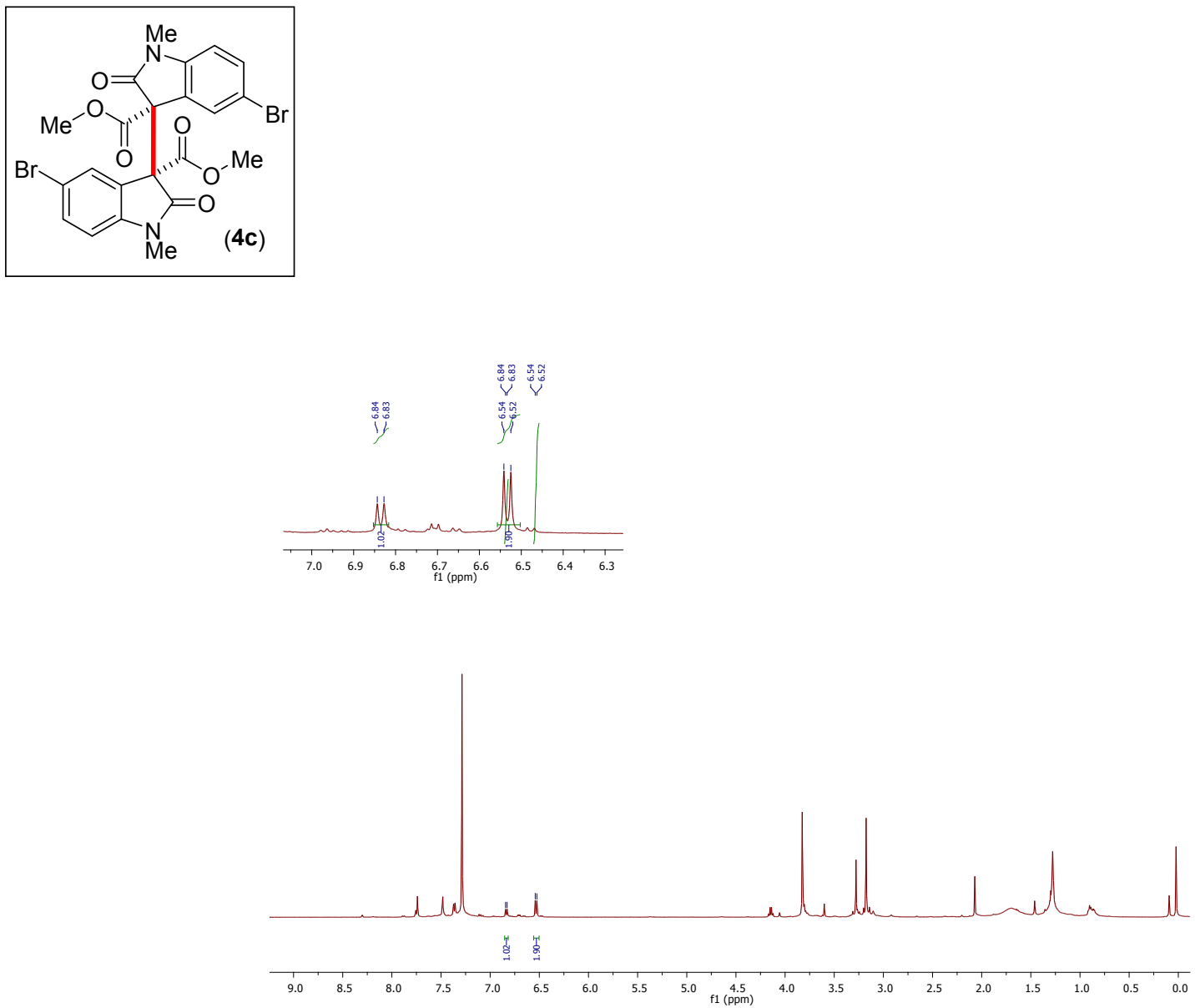

${ }^{1} \mathrm{H}$ NMR $\left(400 \mathrm{MHz}, \mathrm{CDCl}_{3}\right)$ of crude reaction mixture of $(4 \mathrm{c})$ 

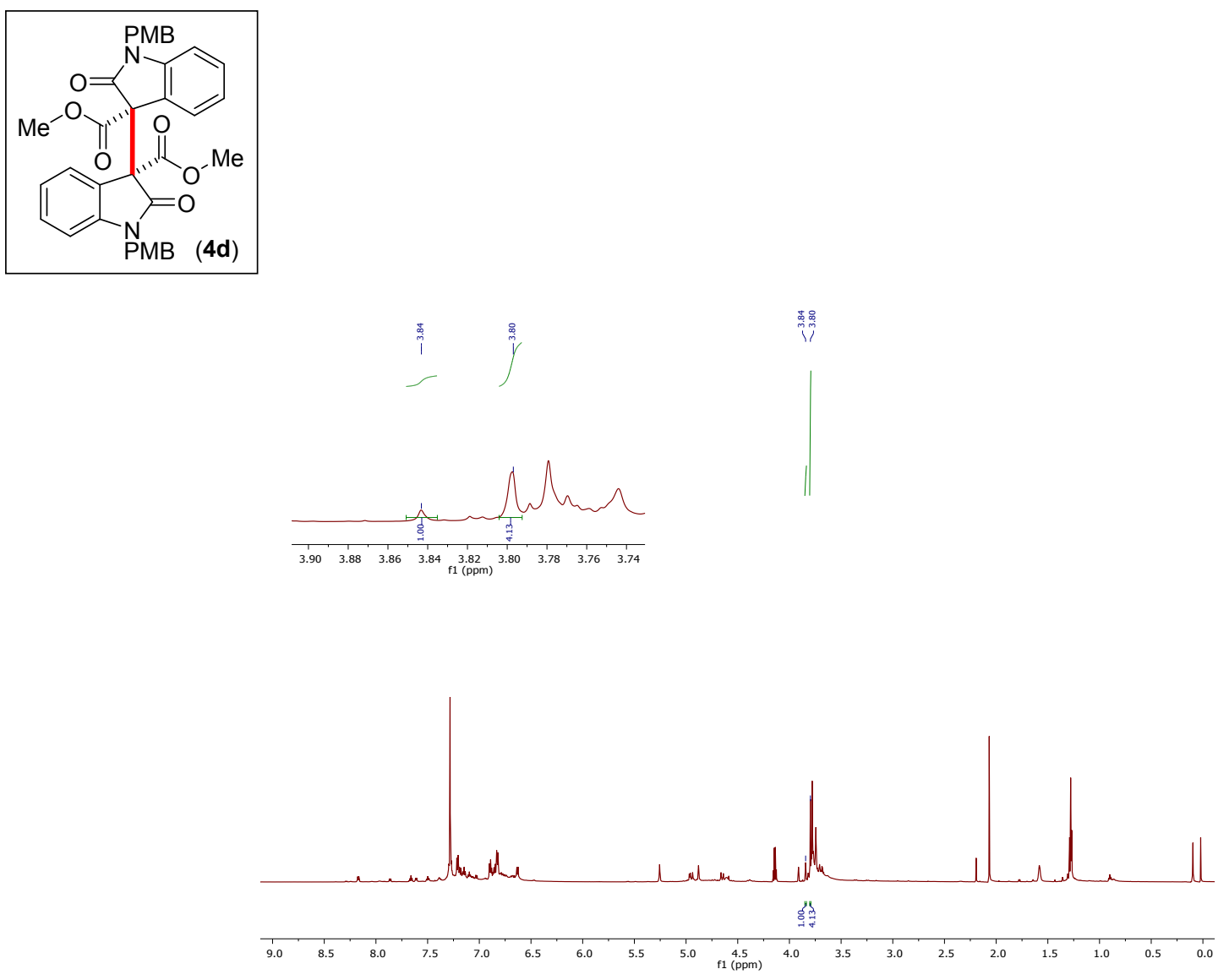

${ }^{1} \mathrm{H}$ NMR (400 MHz, $\mathrm{CDCl}_{3}$ ) of crude reaction mixture of (4d) 

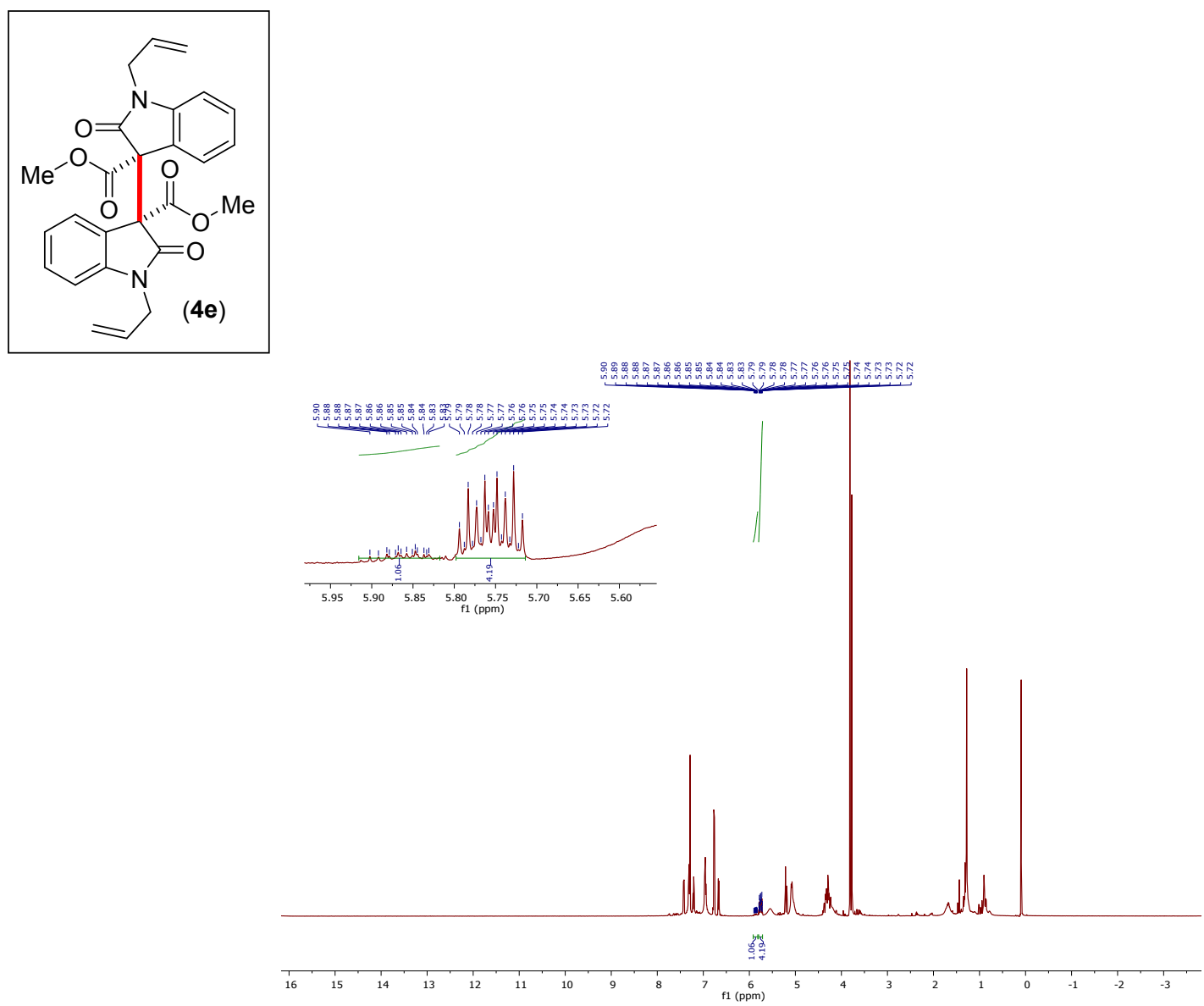

${ }^{1} \mathrm{H}$ NMR (400 MHz, $\mathrm{CDCl}_{3}$ ) of crude reaction mixture of (4e) 

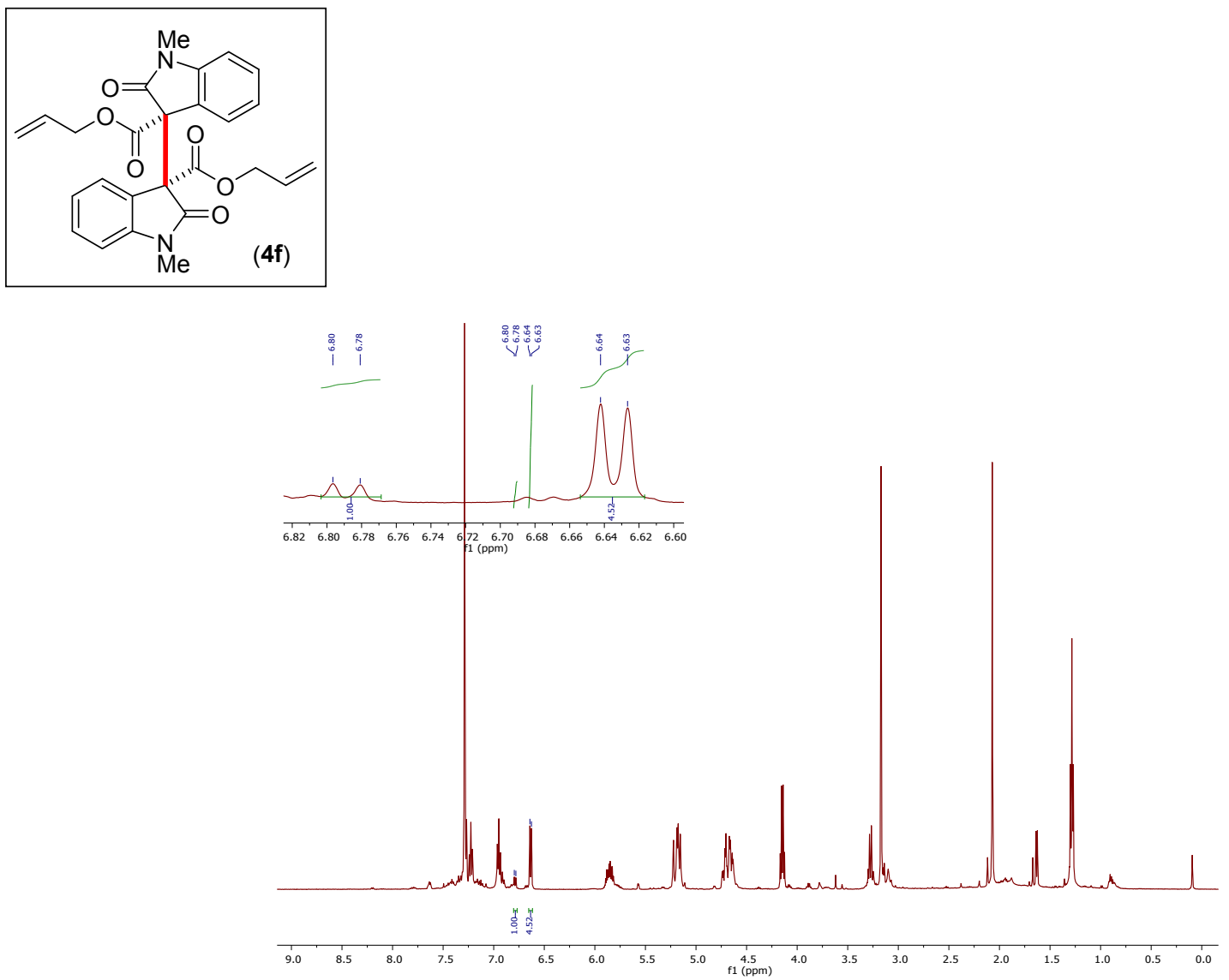

${ }^{1} \mathrm{H}$ NMR $\left(500 \mathrm{MHz}, \mathrm{CDCl}_{3}\right)$ of crude reaction mixture of (4f) 

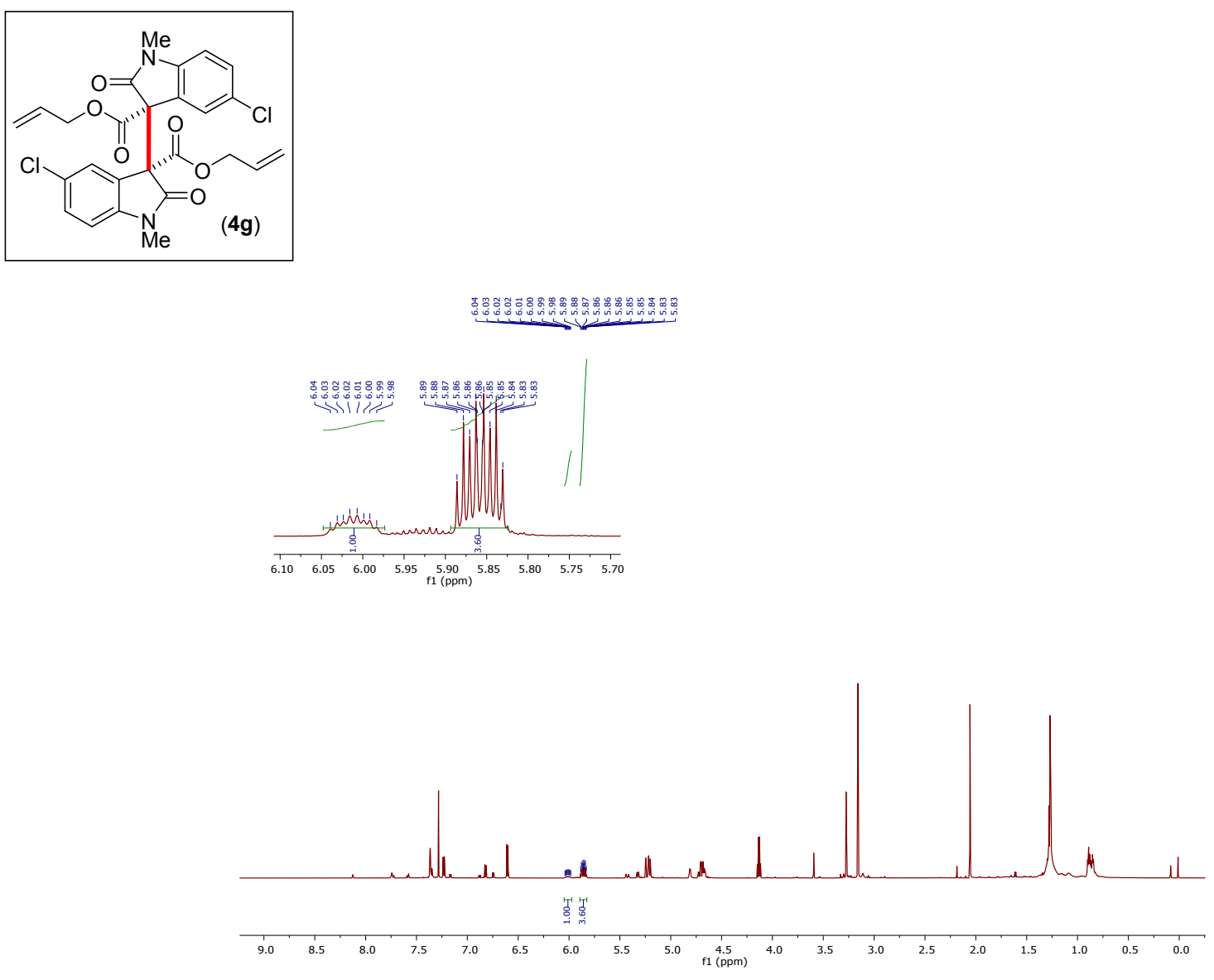

${ }^{1} \mathrm{H} \mathrm{NMR}\left(700 \mathrm{MHz}, \mathrm{CDCl}_{3}\right)$ of crude reaction mixture of $(\mathbf{4 g})$ 


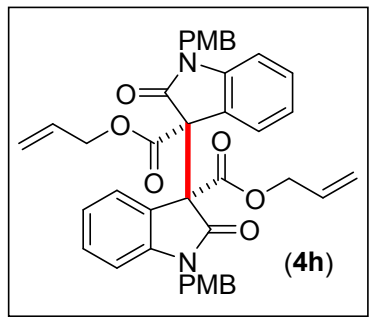

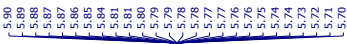
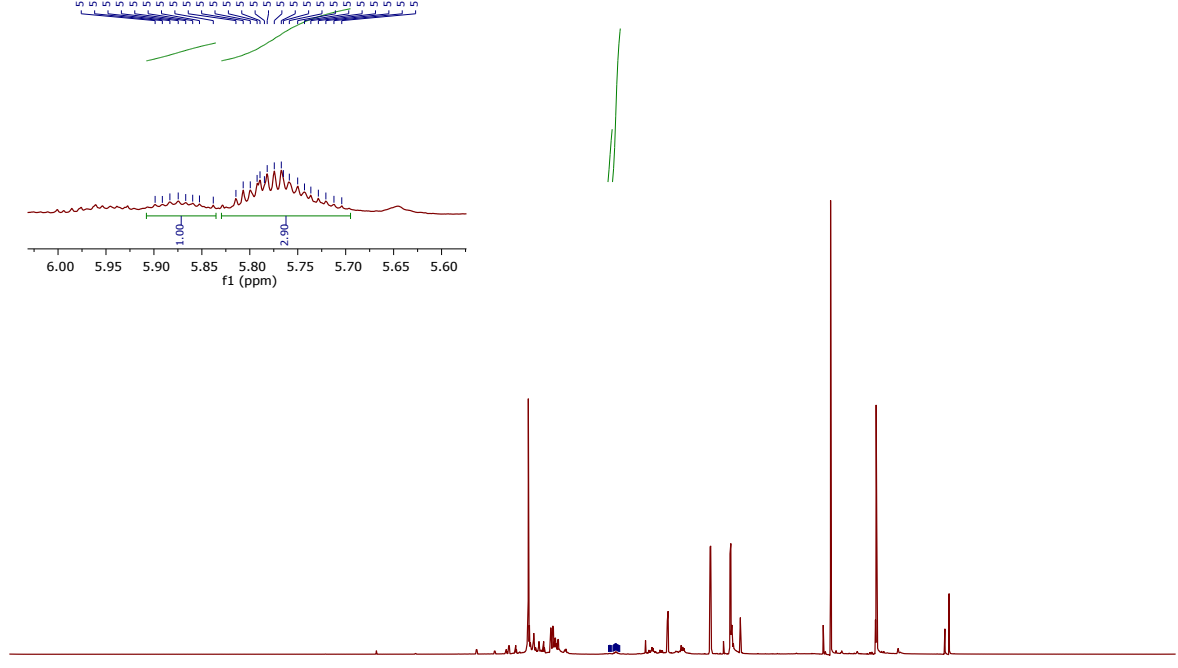

然然

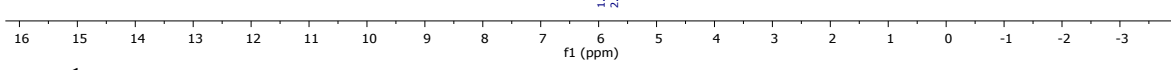

${ }^{1} \mathrm{H} \mathrm{NMR}\left(400 \mathrm{MHz}, \mathrm{CDCl}_{3}\right)$ of crude reaction mixture of $(\mathbf{4 h})$ 

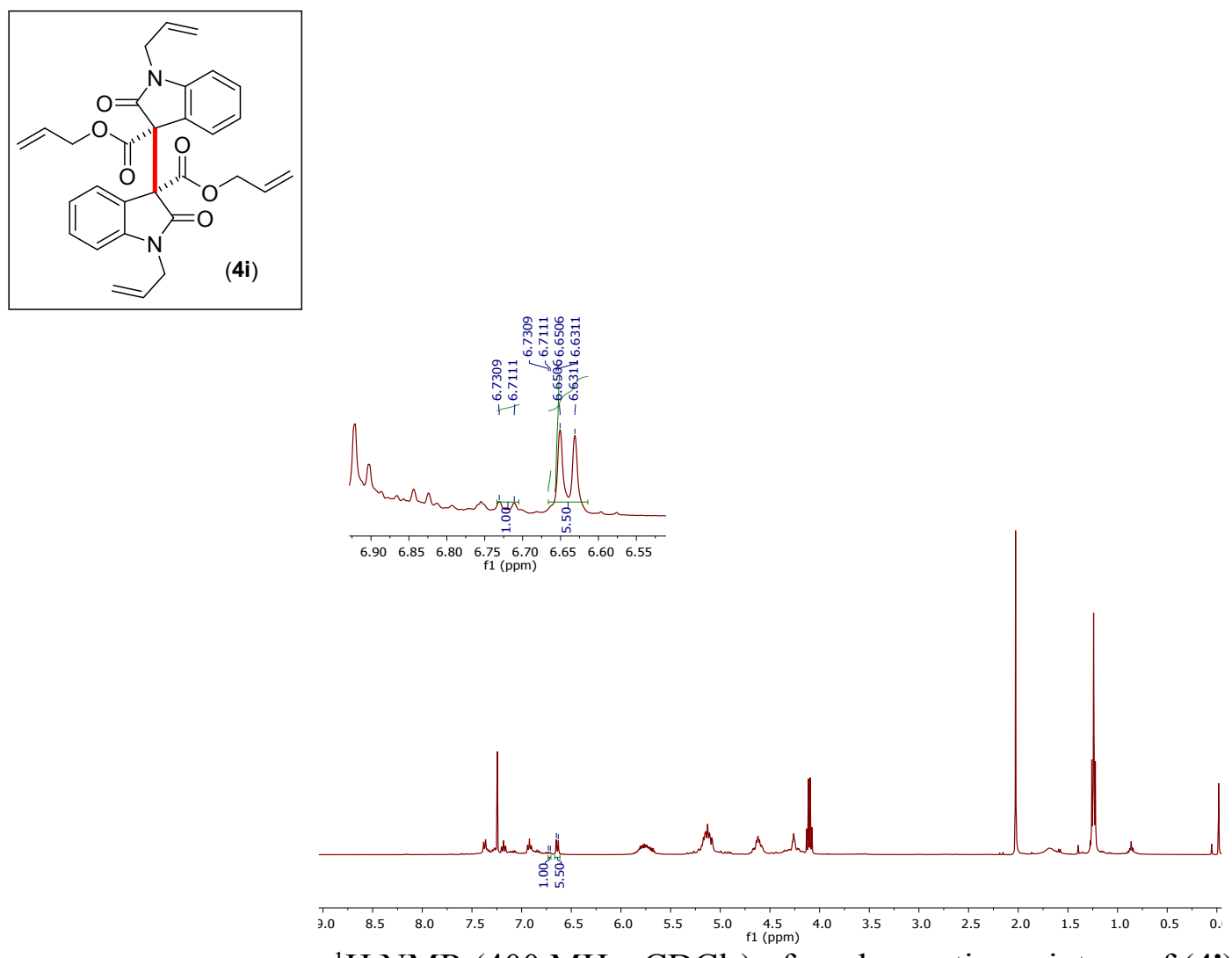

${ }^{1} \mathrm{H} \mathrm{NMR}\left(400 \mathrm{MHz}, \mathrm{CDCl}_{3}\right)$ of crude reaction mixture of (4i) 

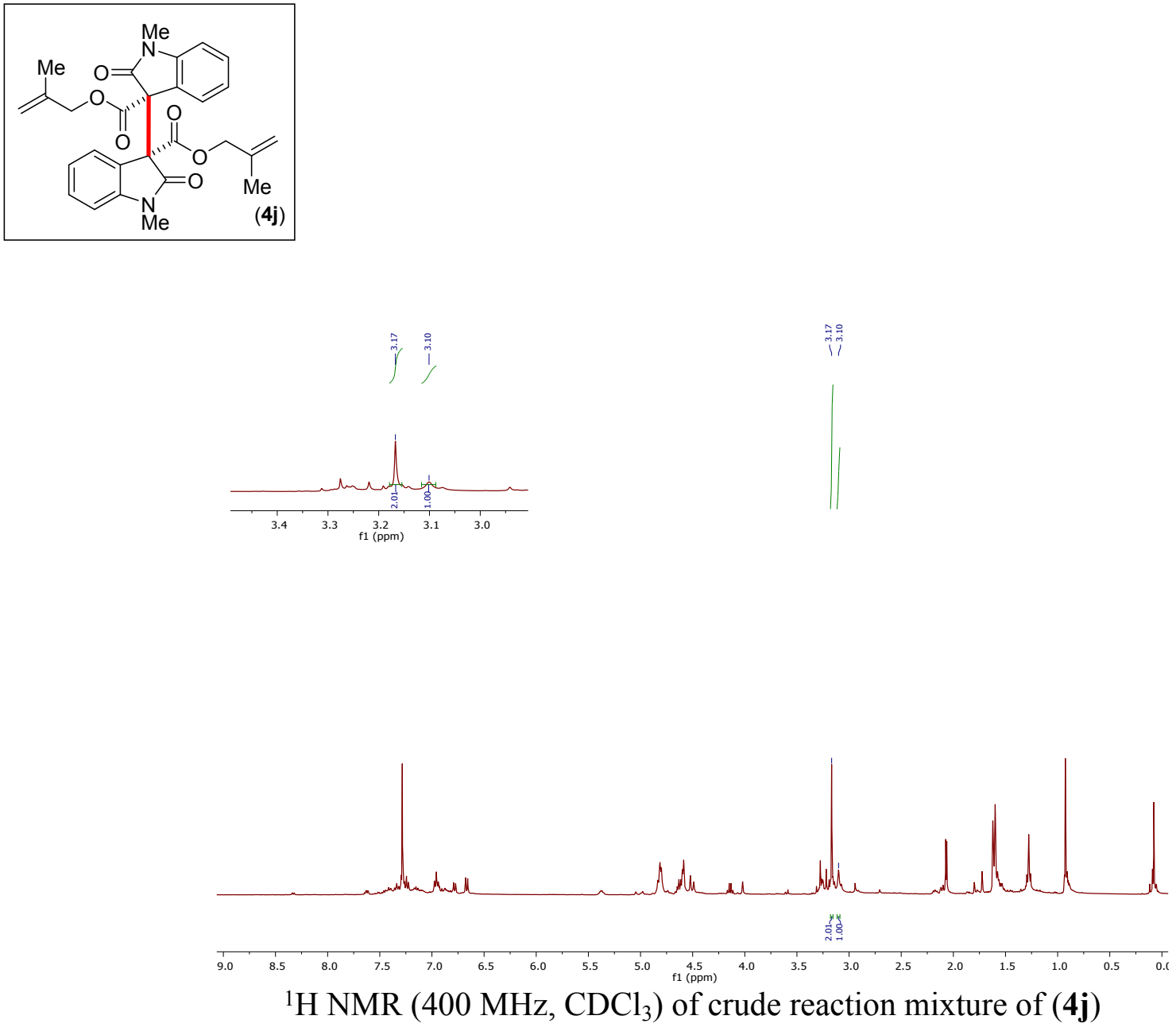

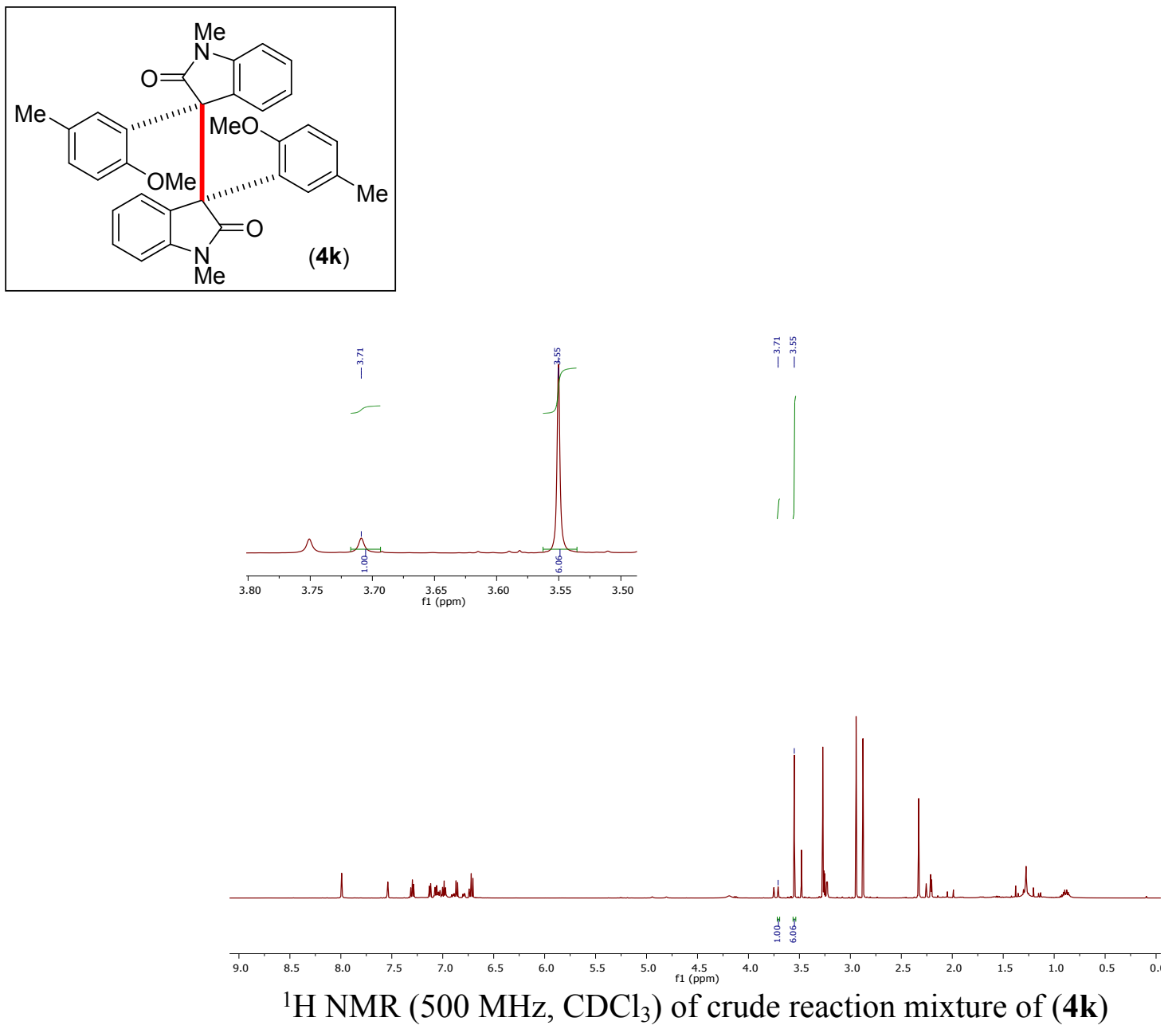

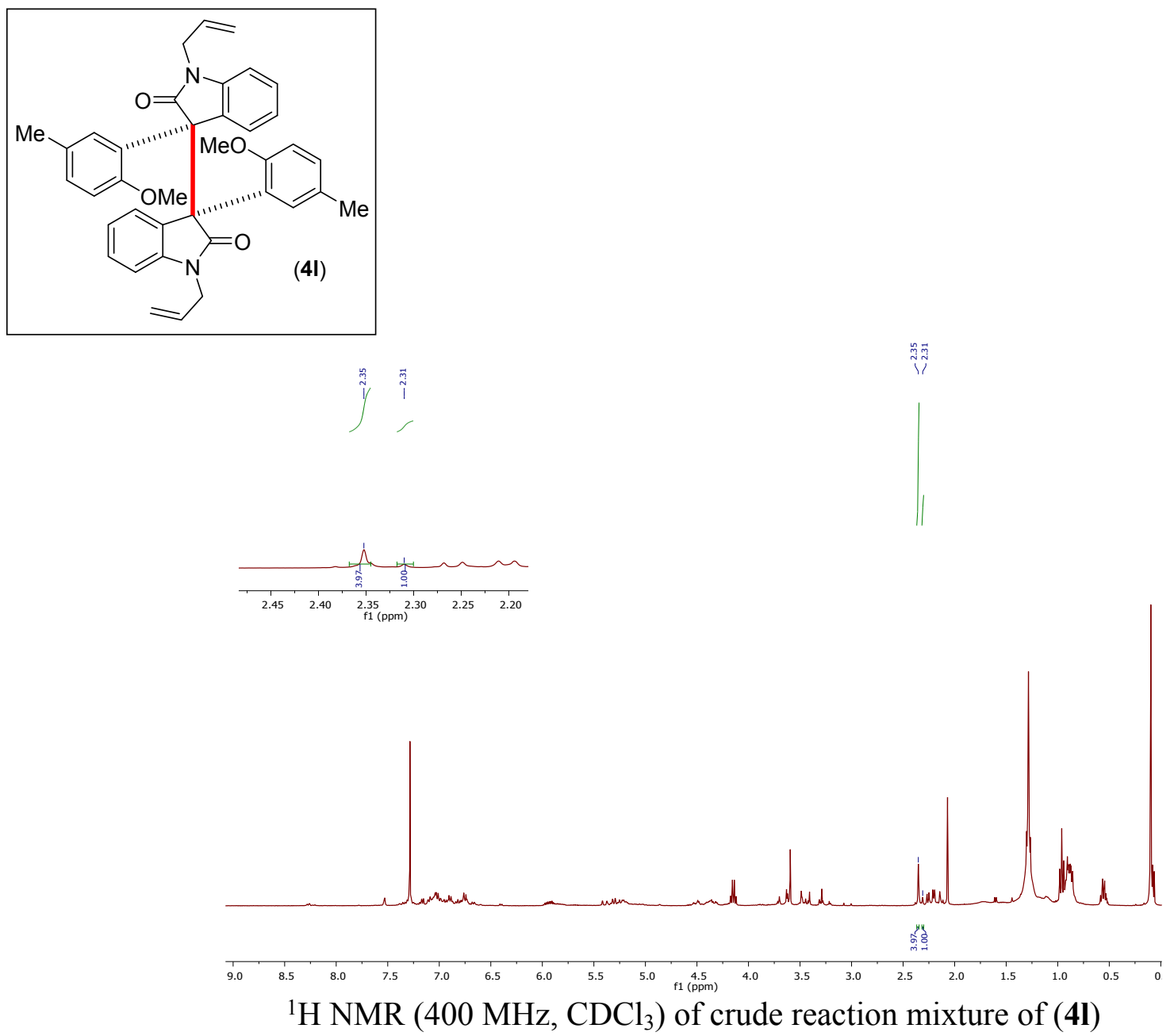

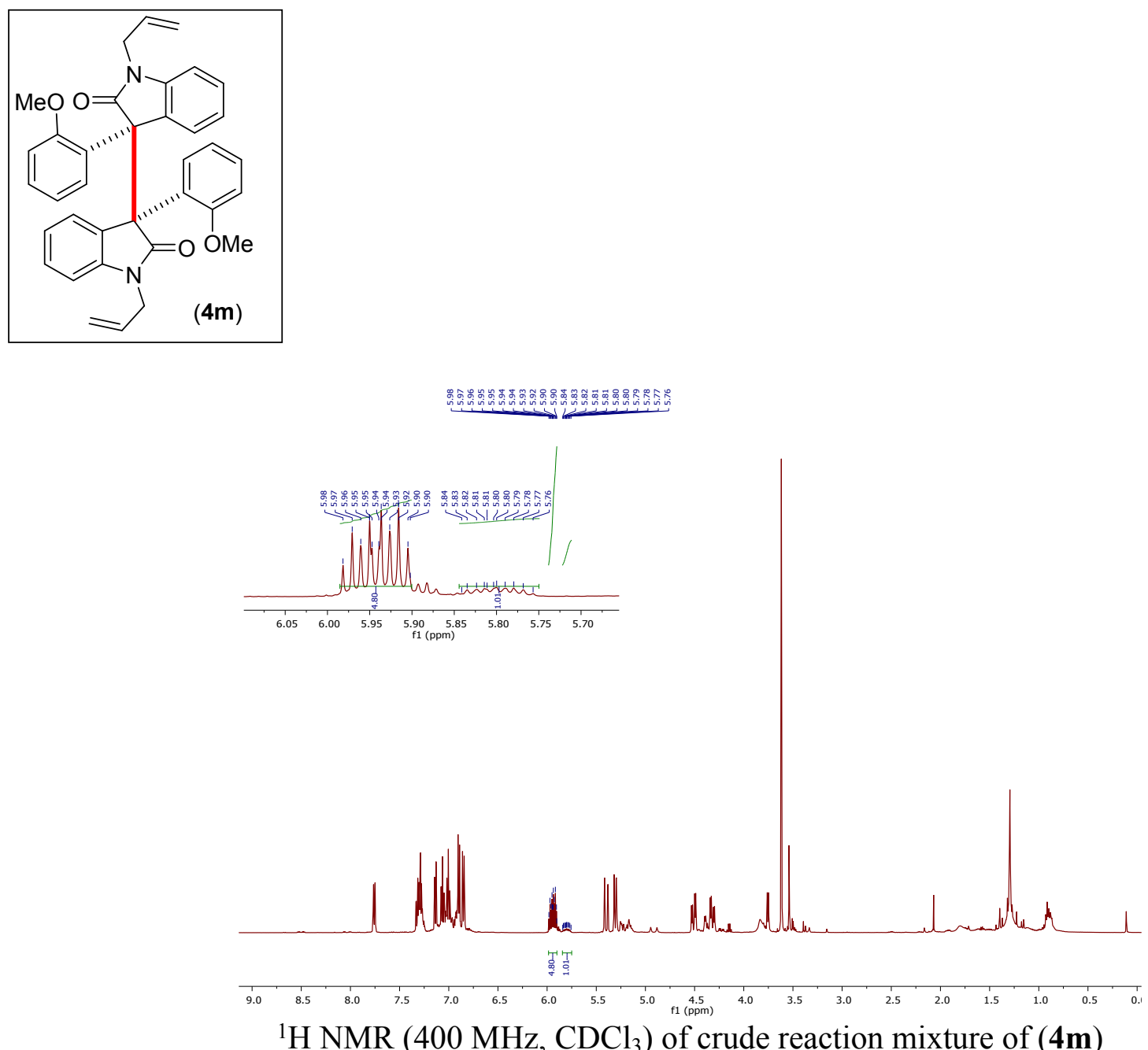

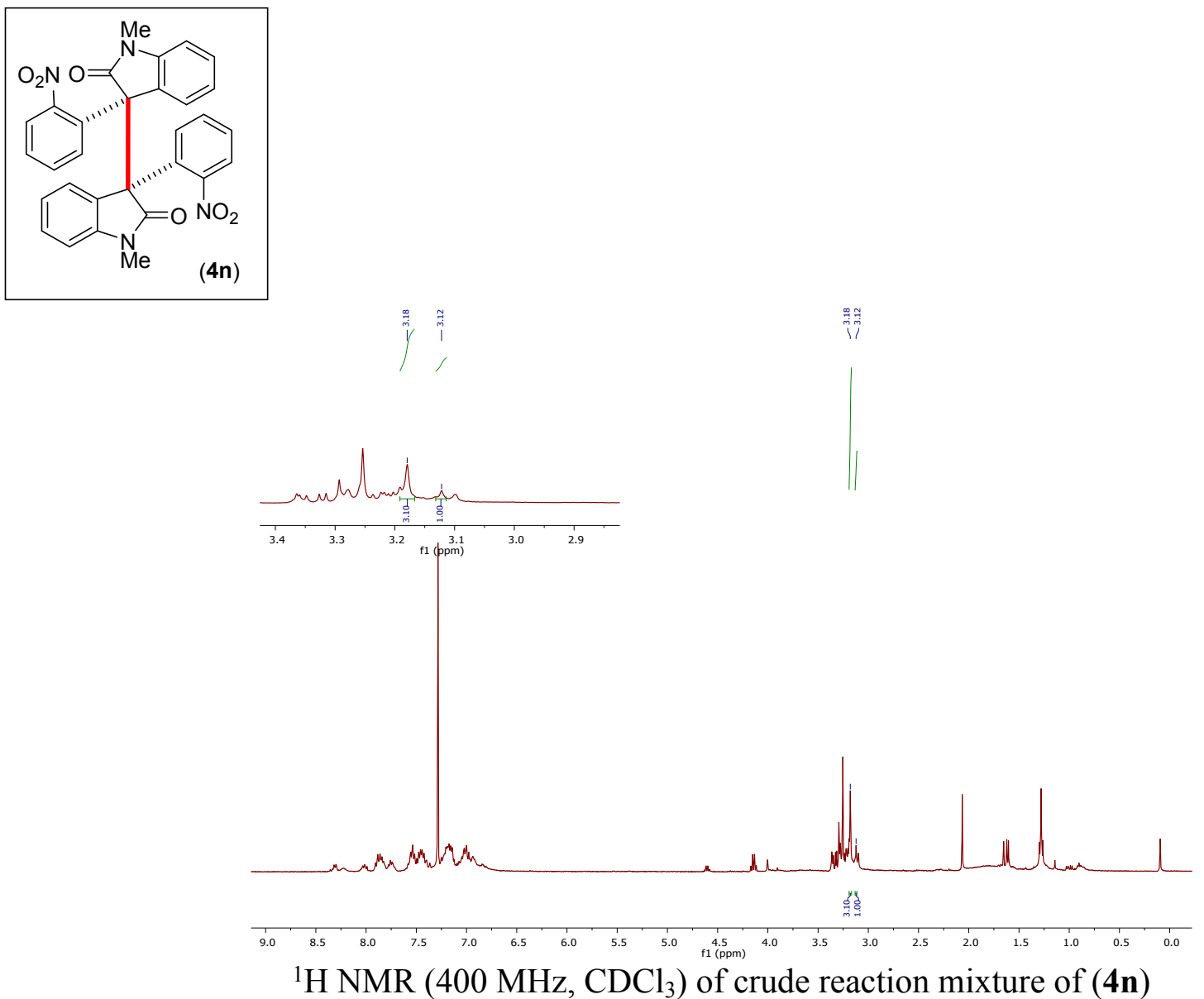

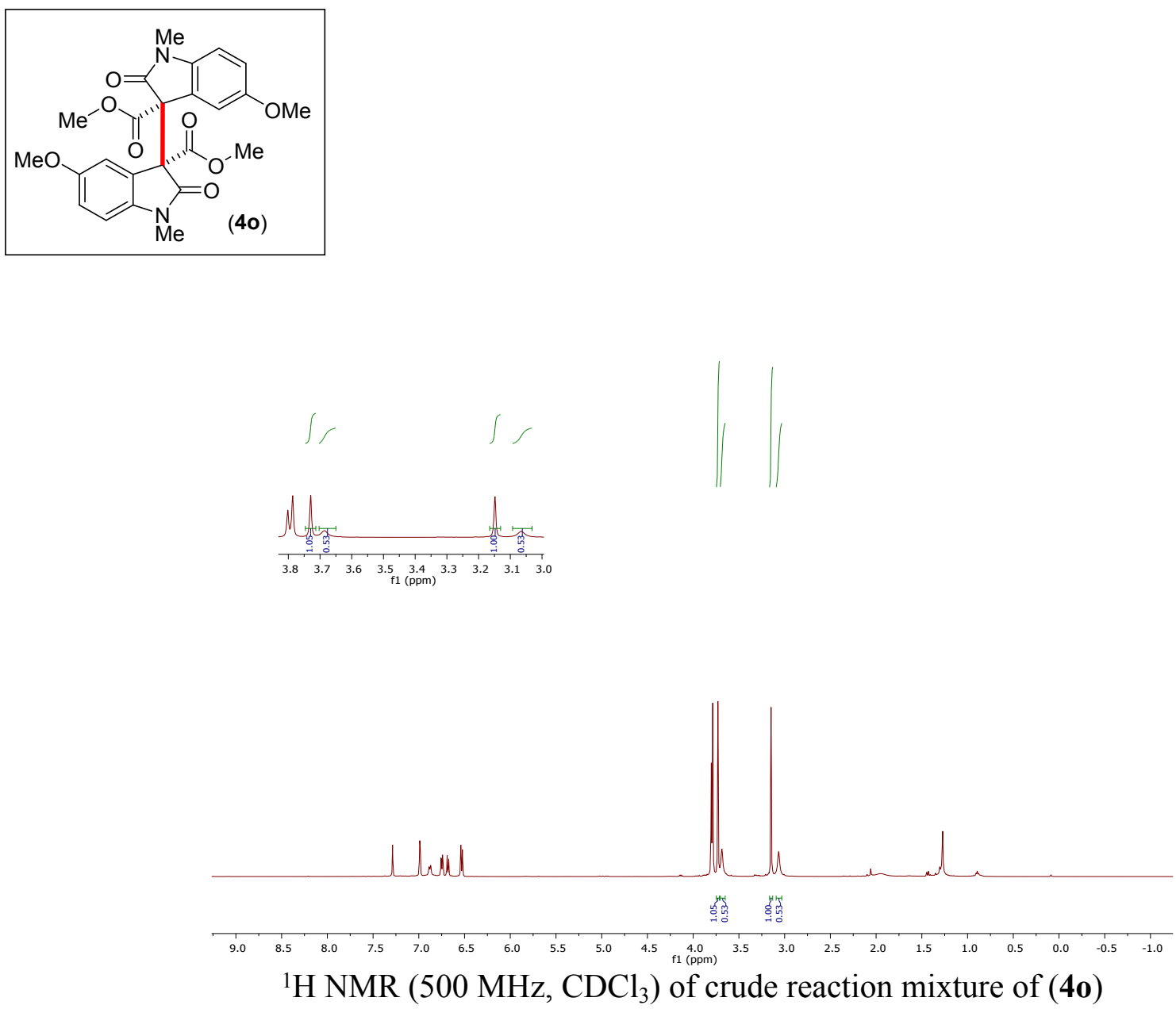

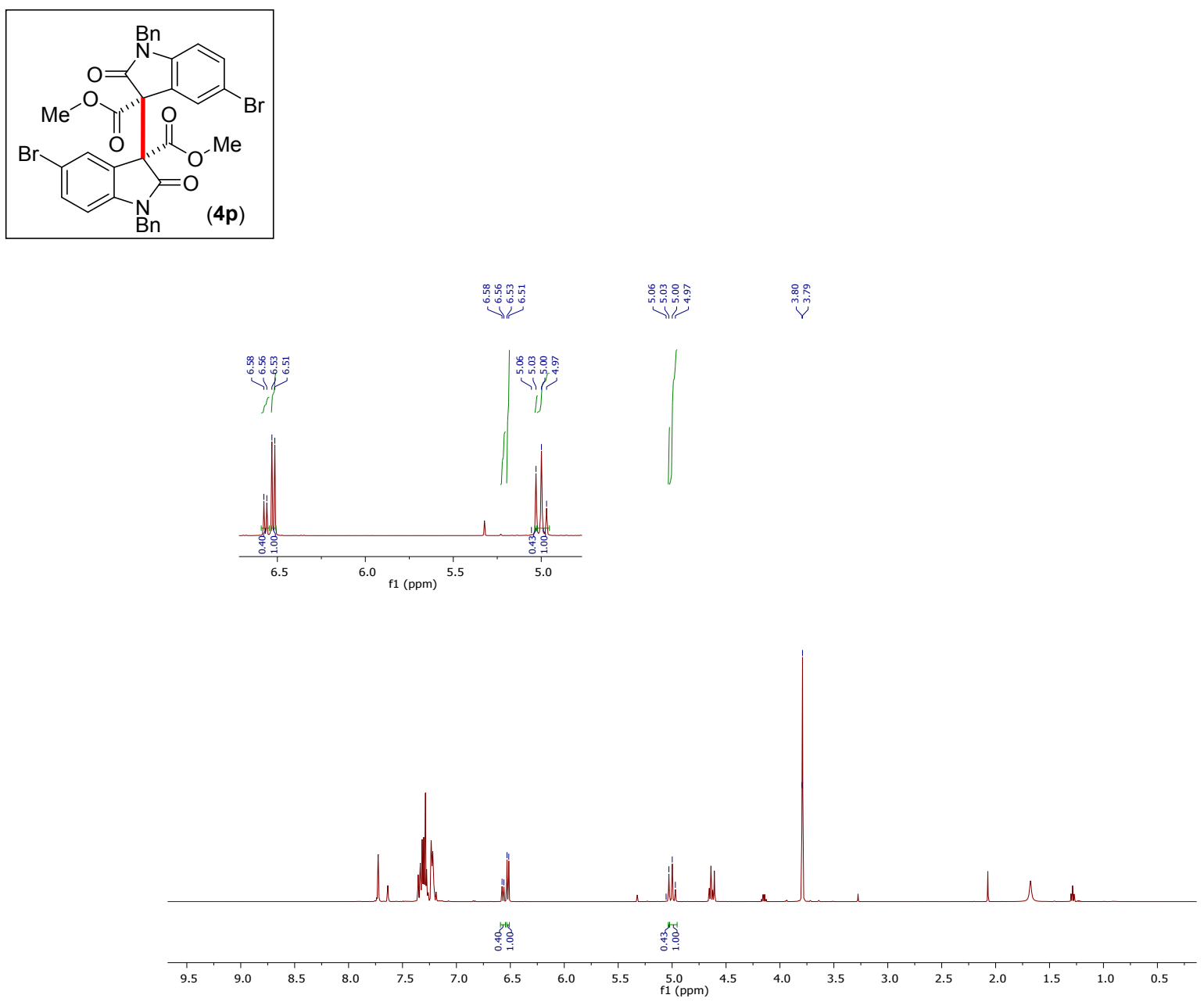

${ }^{1} \mathrm{H}$ NMR (500 MHz, $\left.\mathrm{CDCl}_{3}\right)$ of crude reaction mixture of (4p) 


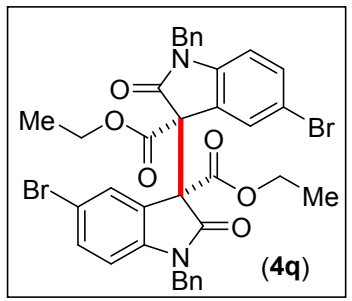

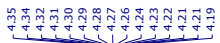
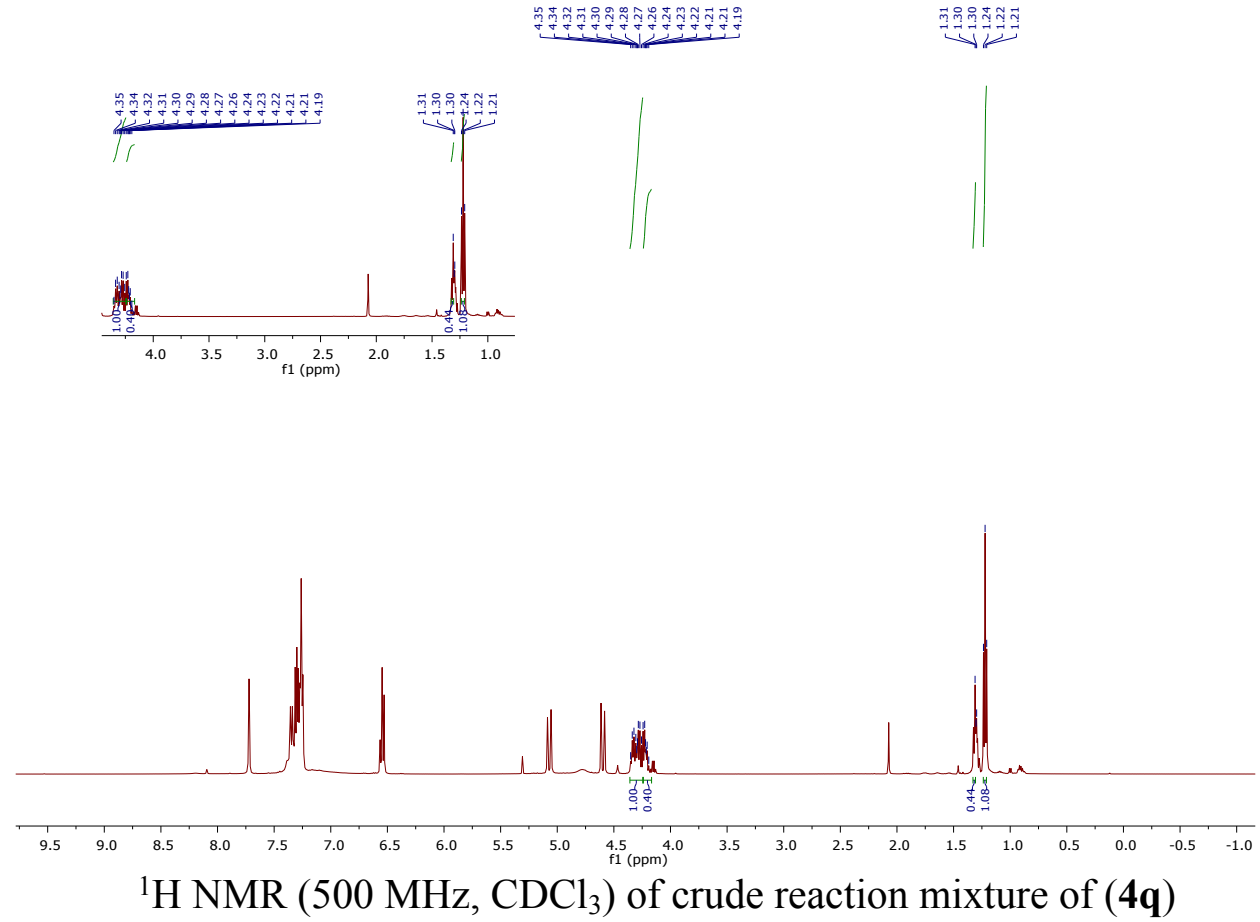

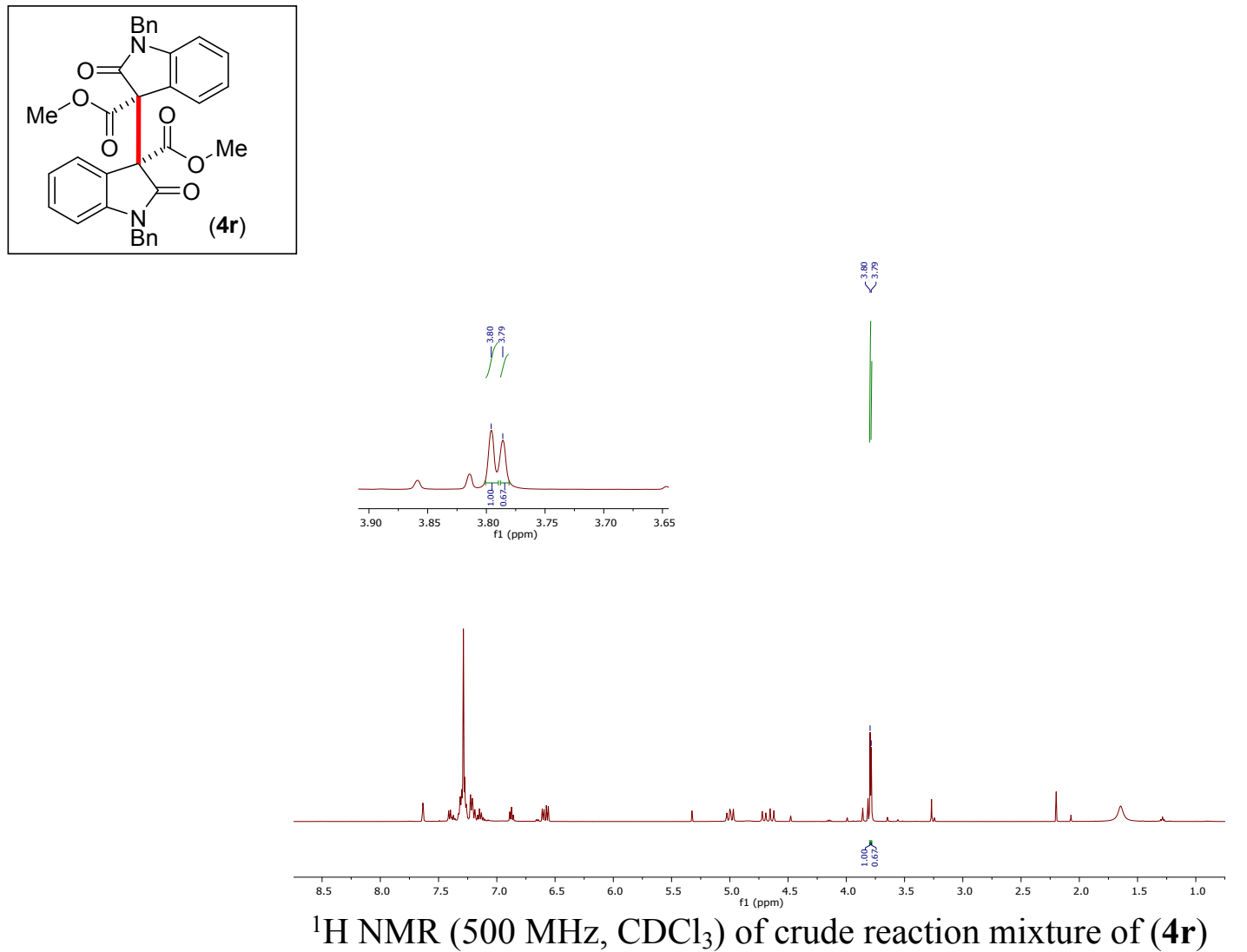

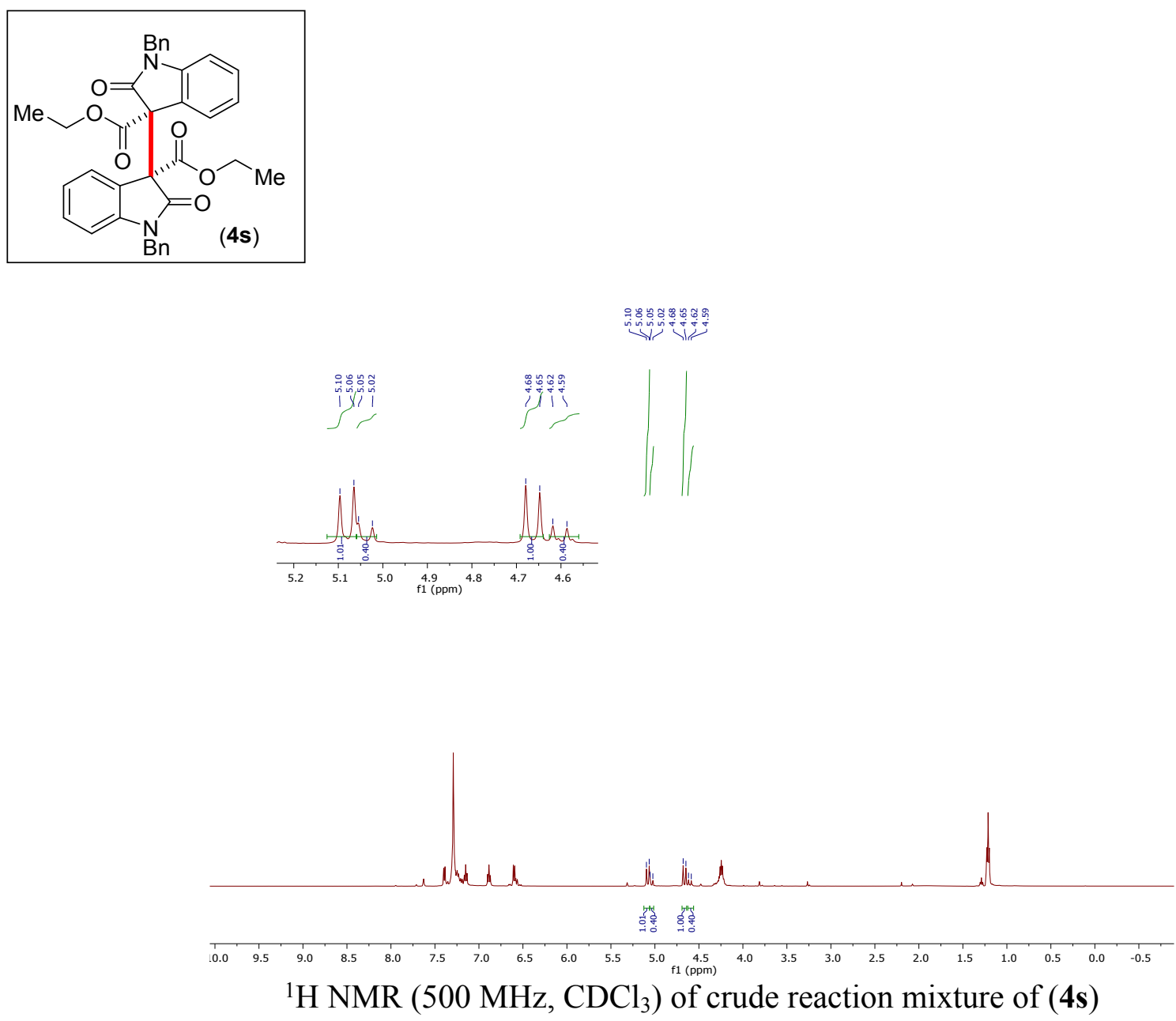

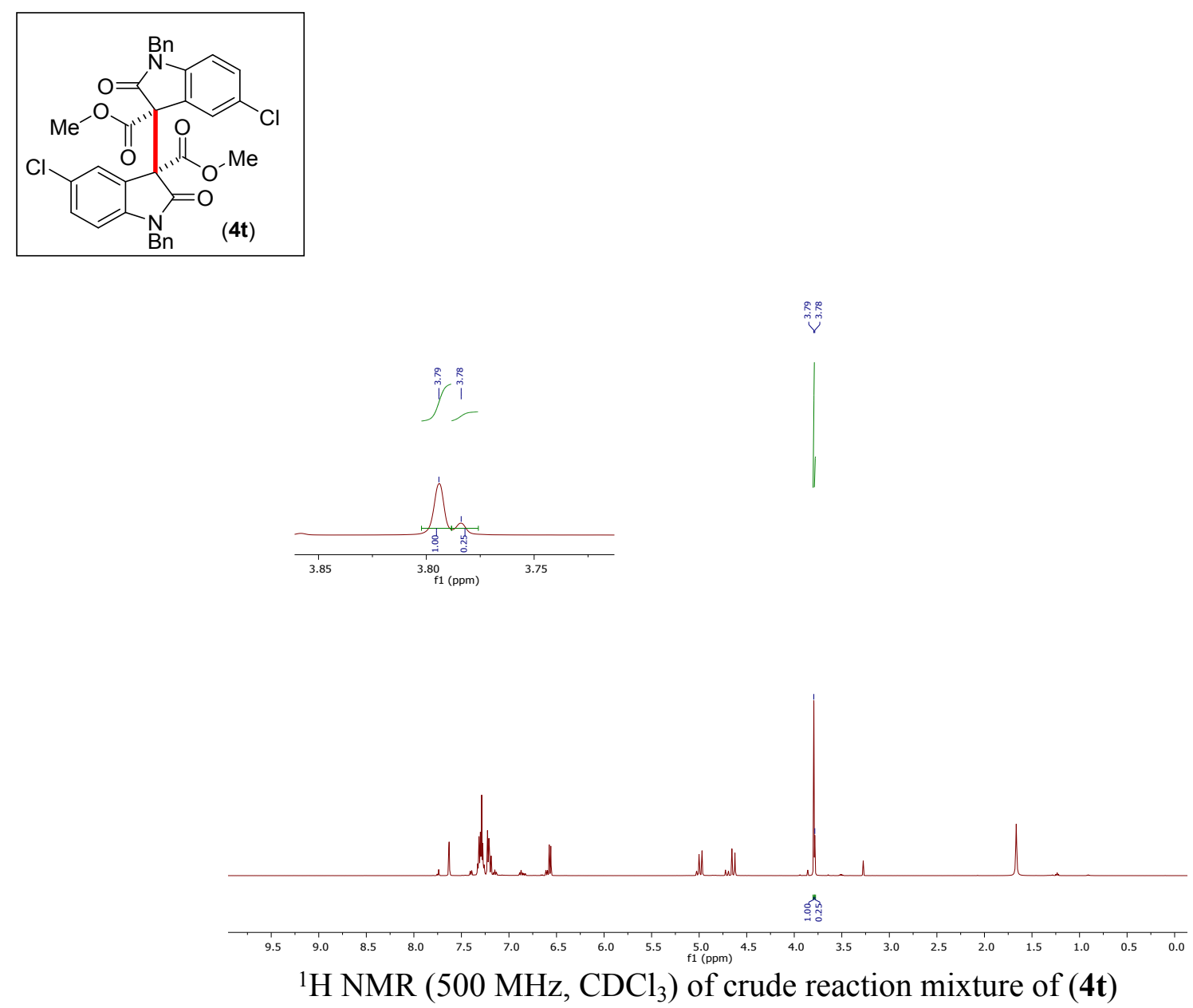

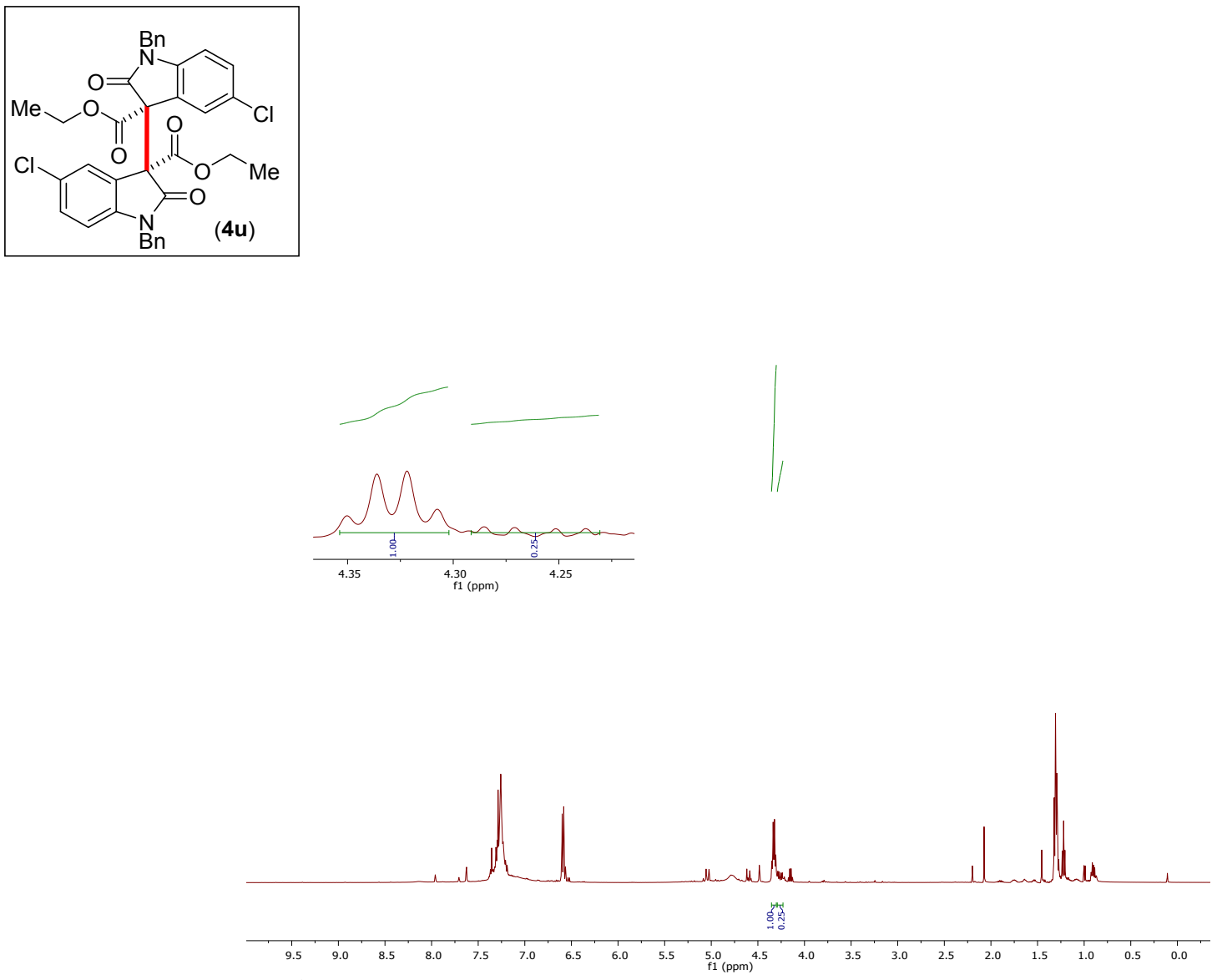

${ }^{1} \mathrm{H} \mathrm{NMR}\left(400 \mathrm{MHz}, \mathrm{CDCl}_{3}\right)$ of crude reaction mixture of $(\mathbf{4 u})$ 